Eugenio Russo

\title{
La presenza degli artefici greco-costantinopolitani a Roma nel VI secolo*
}

In un saggio del 1979 dedicato a F. W. Deichmann e pubblicato nel $1984^{1}$ sostenni una tesi che andava completamente controcorrente, specialmente in relazione al dedicatario, il maggior archeologo cristiano del XX secolo, ch'è stato il massimo sostenitore dell'importanza e dell'azione di Costantinopoli nella diffusione di manufatti e idee, ma non di architetti e maestranze, nel bacino del Mediterraneo: una mobilità d'idee e di sculture, già lavorate e pronte per l'uso, realizzate in marmo del Proconneso, ma un'immobilità degli uomini². Invece a mio parere esistevano elementi tali da suffragare la presenza a Roma (ma il fenomeno già allora aveva un àmbito ben più vasto dello specifico di Roma), accanto a prodotti importati già rifiniti da Costantinopoli ${ }^{3}$, di materiali allo stato di abbozzo e di artefici greco-costantinopolitani, oltre all'attività delle maestranze locali, nel duplice aspetto d'imitatori o di non imitatori dei prodotti greco-costantinopolitani ${ }^{4}$. Tra l'altro tale articolazione, che amplia e arricchisce il quadro, si adegua alla realtà della produzione nelle sue varie fasi di lavorazione e allo stato delle sculture come si son rinvenute nelle cave dell'isola di Proconneso, che oggi conosciamo grazie ai fondamentali contributi di N. Asgari, frutto di un lavoro più che trentennale ${ }^{5}$. E la mobilità

* La presente ricerca è stata effettuata con il parziale contributo dell’Università di Bologna, fondi ex 60\%.

${ }^{1}$ E. Russo, Fasi e nodi della scultura a Roma nel VI e VII secolo, Mélanges de l’École française de Rome. Moyen âge - temps modernes 96, 1, 1984, 7-48.

${ }^{2}$ Rinvio spec., anche con bibl. precedente, a F. W. Deichmann, Ravenna. Hauptstadt des spätantiken Abendlandes I (1969) 63-92; II 1 (1974) e II 2 (1976) passim; II 3 (1989) 273-296. 321-346. Vd. pure, con sfumature diverse, J. P. Sodini, Remarques sur la sculpture architecturale d'Attique, de Béotie et du Péloponnèse à l'époque paléochrétienne, BCH 101, 1977, 423-450; idem, L'artisanat urbain à l'époque paléochrétienne (IVe-VII ${ }^{\mathrm{e}}$ s.), Ktema 4, 1979, 75-78; idem, La sculpture architecturale à l'époque paléochrétienne en Illyricum, in: Actes du X $\mathrm{X}^{\mathrm{e}}$ Congrès international d'archéologie chrétienne (Thessalonique, 28 septembre 4 octobre 1980) I (1984) 207-298; idem in: J. P. Sodini - K. Kolokotsas, Aliki II: La basilique double (1984) 17-209; idem, Le commerce des marbres à l'époque protobyzantine, in: Aa. Vv., Hommes et richesses dans l'empire byzantin, I: IV ${ }^{\mathrm{e}}-\mathrm{VII}{ }^{\mathrm{e}}$ siècle (1989) 163-186; idem, Rome, Constantinople et le Proche Orient: le témoignage de Ravenne, JRA 4, 1991, 403-408; idem in:

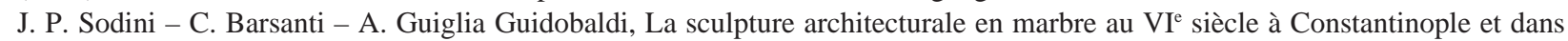
les régions sous influence constantinopolitaine, in: Acta XIII Congressus internationalis archaeologiae christianae (Split - Poreč, 25. 9.-1. 10. 1994) II (1999) 301-315.

${ }^{3}$ Devo sottolineare che io non ho mai pensato né scritto che - come invece mi attribuiscono C. Barsanti e A. Guiglia Guidobaldi in: F. Guidobaldi - C. Barsanti - A. Guiglia Guidobaldi, San Clemente. La scultura del VI secolo (1992) 256 - nel VI sec. le maestranze greco-costantinopolitane abbiano scolpito a Roma »le lastre e i pilastrini della recinzione clementina - ed in seguito quelle delle altre chiese« di Roma: come se io ritenessi che nessuno dei prodotti greco-costantinopolitani presenti nella città fosse stato importato già rifinito da Costantinopoli. Ho invece indicato chiaramente le opere eseguite a mio parere a Roma da artefici greco-costantinopolitani, come per S. Clemente, quelle in cui lasciavo aperta la possibilità d'una lavorazione a Roma o d'un'importazione diretta dei prodotti già rifiniti (»presenza di maestranze e/o prodotti greco-cost/i«: Russo [nota 1] 19. 23-24), quelle che già allora mi apparivano prodotti importati già rifiniti (»prodotti standard«: ibidem 19-22). Evidentemente non si è letto bene. Ancor peggio ha letto A. Melucco Vaccaro, s. v. Roma, in: Enciclopedia dell'arte medievale X (1999) 98-99 e eadem, Le botteghe dei lapicidi: dalla lettura stilistica all'analisi delle tecniche di produzione, in: Roma nell'alto medioevo. XLVIII Settimana di studio del Centro italiano di studi sull'alto medioevo, 27 aprile - 1 maggio 2000 (2001) 396-399, la quale mi attribuisce »il tentativo di ricostruire a R. una bottega di artisti greco-costantinopolitani attiva dall'età teodoriciana fino a Gregorio Magno, alla quale sarebbero da attribuire, lungo una linea di continuità e di radicamento urbano, sia le transenne di S. Clemente sia i resti di plutei di modulo geometrico delle Grotte vaticane, interpretati come resti delle recinzioni gregoriane«: un fraintendimento grave e gratuito.

${ }^{4}$ Russo (nota 1) 9-37.

${ }^{5}$ La bibl. dei contributi specifici sull'argomento di N. Asgari è riportata in: E. Russo, Introduzione allo studio della scultura architettonica e decorativa bizantina in Turchia, Bizantinistica n. s. 6, 2004, nota 9 (in corso di stampa). 


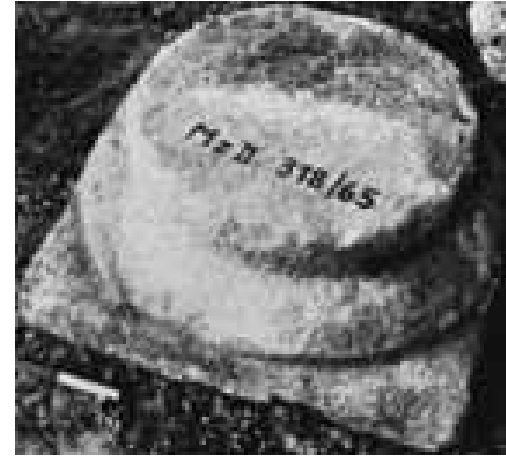

1 Siracusa, Museo archeologico, deposito: una delle basi del carico naufragato presso Marzamemi degli artefici, oltre che dei prodotti, è stata verificata nel corso degli anni, a parte Roma ${ }^{6}$, in Palestina ${ }^{7}$, a Ravenna e nel suo territorio ${ }^{8}$, nel bacino superiore dell'Adriatico ${ }^{9}$, in Liguria $^{10}$, e persino a Efeso ${ }^{11}$ e a Nicea ${ }^{12}$. Nel 1979 ho anche coniato il termine 'greco-costantinopolitano', per indicar non soltanto le maestranze della capitale, ma anche quelle del mondo greco che dal punto di vista artistico faceva riferimento a Costantinopoli.

Ritorno sull'argomento a molti anni dalla pubblicazione di quel saggio perché la mia proposta del 1984 non è stata accettata, a volte nemmeno ben compresa: si è detto che le sculture di S. Clemente son state importate quasi rifinite ${ }^{13} \mathrm{o}$ rifinite ${ }^{14}$, o addirittura in un unico invio, pronte per l'uso, assieme al materiale delle altre chiese romane, all'epoca di Giovanni II (533-535) ${ }^{15}$; e da ultimo si è pensato »ad un invio decisamente cospicuo o, forse, a più invii successivi « ${ }^{16}$, ma »ravvicinati nel tempo«, »con tutta probabilità precedente alle guerre gotiche ${ }^{17}$. Ci si rifaceva infatti, $\mathrm{e}$

${ }^{6}$ E. Russo, La recinzione del presbiterio di S. Pietro in Vaticano dal VI all’VIII secolo, RendPontAc 55/56, 1982/83-1983/84 (1985) 3-33; idem, Due frammenti, la filologia, una chiesa: ricomposto un pluteo di S. Clemente a Roma, in: Studi in memoria di Giuseppe Bovini (1989) 597-610; idem, Apparati decorativi, in: Aurea Roma. Dalla città pagana alla città cristiana (Catalogo della Mostra, Roma, 22 dicembre 2000 - 20 aprile 2001) (2000) 191-199 e 644-646; idem in: Ecclesiae Urbis. Atti del Congresso internazionale di studi sulle chiese di Roma, IV-X secolo, Roma, 4-10 settembre 2000, Studi di antichità christiana 59 (2003) 1629 -1632.

7 E. Russo, La scultura del VI secolo in Palestina. Considerazioni e proposte, ActaAArtHist s. a. 6, 1987, 113-248.

${ }^{8}$ E. Russo, I monumenti di Pesaro dal secolo VI all'epoca romanica, in: Pesaro tra Medioevo e Rinascimento (1989) 105-111; idem, Una fronte frammentaria d'altare paleocristiano rinvenuta nella cattedrale di Parma, RACr 67, 1991, 383-404; idem, »Ravenna, Hauptstadt des spätantiken Abendlandes« di Friedrich Wilhelm Deichmann, Vetera Christianorum 29, 1992, 137-160; idem, Archeologia e storia dell'arte a Ravenna fino al VI secolo d. C., Ravenna studi e ricerche 4, 2, 1997, 127-146; idem, L'architettura di Ravenna paleocristiana (2003); idem in: Dizionario di iconografia e arte cristiana (2004) 1108-1112 s. v. Ravenna.

${ }^{9}$ E. Russo, Sculture del complesso eufrasiano di Parenzo (1991); idem, Una riflessione sull'episcopio di Parenzo, Bizantinistica n. s. 2, 2000, 179-195.

${ }^{10}$ E. Russo, La scultura in Liguria dal VI all’VIII secolo. Stimoli per un approfondimento, in: Romana pictura e Christiana signa. Due mostre a confronto. Arte figurativa in Liguria fra età imperiale e altomedioevo. Atti delle Giornate di studio, Genova, 12-13 dicembre 1998 (2003) 73-77 e 104-106.

${ }^{11}$ E. Russo, La scultura a Efeso in età paleocristiana e bizantina. Primi lineamenti, in: R. Pillinger et al. (edd.), Efeso paleocristiana e bizantina - Frühchristliches und byzantinisches Ephesos. Atti del Convegno internazionale di studi, Roma, 22-24 febbraio 1996, AForsch 3 (1999) 26-53; idem, Sculture paleocristiane e bizantine dell’Artemision di Efeso, in: U. Muss (ed.), Der Kosmos der Artemis von Ephesos, SoSchrÖAI 37 (2001) 265-278.

${ }^{12}$ E. Russo, Considérations sur la sculpture architecturale et décorative à Nicée à l'époque paléochrétienne, Bizantinistica n. s. 4, 2002, 1-11.

${ }^{13}$ P. Pensabene, La decorazione architettonica, l'impiego del marmo e l'importazione di manufatti orientali a Roma, in Italia e in Africa (II-VI d. C.), in: Società romana e impero tardoantico III: Le merci, gli insediamenti (1986) 293; J. P. Sodini, Le commerce des marbres dans la Méditerranée (IV e-VII s.), in: V Reunió d’arqueologia cristiana hispànica (Cartagena, 16-19 abril 1998) (2000) 434.

${ }^{14}$ Pensabene (nota 13) 350. 357; Deichmann (nota 2:1989) 321-322; Melucco Vaccaro (nota 3:1999) 98 (»sicura origine costantinopolitana«); L. Paroli, La scultura a Roma tra il VI e il IX secolo, in: Roma dall'antichità al medioevo. Archeologia e storia nel Museo Nazionale Romano - Crypta Balbi (2001) 132 (»provenienti dalle officine imperiali del Proconneso«); A. Guiglia Guidobaldi, Pluteo di recinzione frammentario, in: Aa. Vv., Christiana loca. Lo spazio cristiano nella Roma del primo millennio II (2001) 202-203 figg. a p. 202 (»materiali importati da Costantinopoli«); eadem, La scultura di arredo liturgico nelle chiese di Roma: il momento bizantino, in: Ecclesiae Urbis (nota 6) 1483-1488 figg. 1-2.

${ }^{15}$ C. Barsanti - A. Guiglia Guidobaldi in: Guidobaldi - Barsanti - Guiglia Guidobaldi (nota 3) 258-261: le due studiose ipotizzano esplicitamente per »tutto il materiale romano« di S. Clemente, dei Ss. Cosma e Damiano, di S. Maria in Cosmedin, S. Saba, S. Lorenzo f. l. m., S. Martino ai Monti, abbazia delle Tre Fontane, S. Cecilia, S. Agata dei Goti, S. Stefano Rotondo, S. Maria in Trastevere, S. Prisca, S. Maria in Via Lata, S. Giovanni in Laterano, di Albano, che »nella sua totalità - e quindi non solo le sculture della recinzione di S. Clemente - abbia fatto parte di un medesimo invio«, all'epoca di Giovanni II (533-535). L'ipotesi è ripresa da Paroli (nota 14) 133.

${ }^{16}$ A. Guiglia Guidobaldi, Gli arredi liturgici: botteghe e produzioni, in: Christiana loca. Lo spazio cristiano nella Roma del primo millennio (Catalogo della Mostra, Roma, 5 settembre - 15 novembre 2000) (2000) 267-269 (prima delle guerre gotiche).

17 A. Guiglia Guidobaldi in: Ecclesiae Urbis (nota 6) 1483. 1489-1490. Cf. F. Guidobaldi, Strutture liturgiche negli edifici cristiani di Roma dal IV al VII secolo, in: Aa. Vv., Materiali e tecniche dell'edilizia paleocristiana a Roma (2001) 190 (»plutei d’importazione costantinopolitana«, »subito prima e subito dopo le guerre gotiche«). 
ci si rifà ancora, acriticamente, al ritrovamento del carico della nave naufragata presso Marzamemi in Sicilia ${ }^{18}$, con le sue sculture architettoniche di una basilica prefabbricata e probabilmente dirette in Africa, per applicar indiscriminatamente la realtà di Marzamemi a S. Clemente e a tutta la gamma della produzione in marmo proconnesio disseminata nel bacino del Mediterraneo e del mar Nero ${ }^{19}$, senza distinguere caso per caso.

Credo d'esser uno dei pochi che han avuto la possibilità d'esaminare di persona i marmi di Marzamemi, i quali giacciono in un deposito all'aperto del Museo archeologico di Siracusa. Ebbene i pezzi sono sì finiti, ma non tutti, giacché nei capitelli corinzii ${ }^{20}$ la parte superiore del $k a-$ lathos e l'abaco con il marchio di fabbrica in lettere greche sono solo semilavorati ${ }^{21}$ : si potevano anche metter in opera in quello stato, ma è più probabile che sul posto fossero completati. Pure le basi delle colonne (fig. 1) ${ }^{22}$ sono soltanto sbozzate, semilavorate. Ora il fenomeno dell'impiego di basi soltanto sbozzate, come giungevano dalle cave del Proconneso, è diffuso ${ }^{23}$, e a riprova richiamo il S. Giovanni di Efeso ${ }^{24}$ e il S. Pantaleone di Afrodisia in Cilicia ${ }^{25}$, dove assistiamo a una mescolanza di basi in parte allo stato di abbozzo in parte lavorate in loco e rifinite, e le sigle di fabbrica con cui le basi di S. Giovanni sono contrassegnate eran destinate a scomparire man mano che i pezzi venivan lavorati, e potevan restare solamente quelle legate alla costruzione, sulla superficie superiore, o nei bordi dei plinti. A mio parere ha ragione Pallas a vedere nel fenomeno dell'impiego di basi soltanto sbozzate una sorta di rivoluzione estetica ${ }^{26}$, mentre non convince la »necessità economica « invocata da J.P. Sodini ${ }^{27}$, dato che a Marzamemi soltanto le basi sono sbozzate, e ciò non può certo spiegarsi con la necessità economica. A Marzamemi nemmeno le colonne eran del tutto rifinite alle estremità ${ }^{28}$, a smentire che non fosse necessaria un'opera di rifinitura e di adattamento sul posto persino per un carico di sculture ch'è registrato comunemente dagli studiosi con la qualifica di compiutezza.

Quando intrapresi le ricerche sull'argomento negli anni '70, avevo la convinzione che gli artefici greco-costantinopolitani che operavan fuori sede non avessero la capacità tecnica di eseguire trafori, e che le transen-

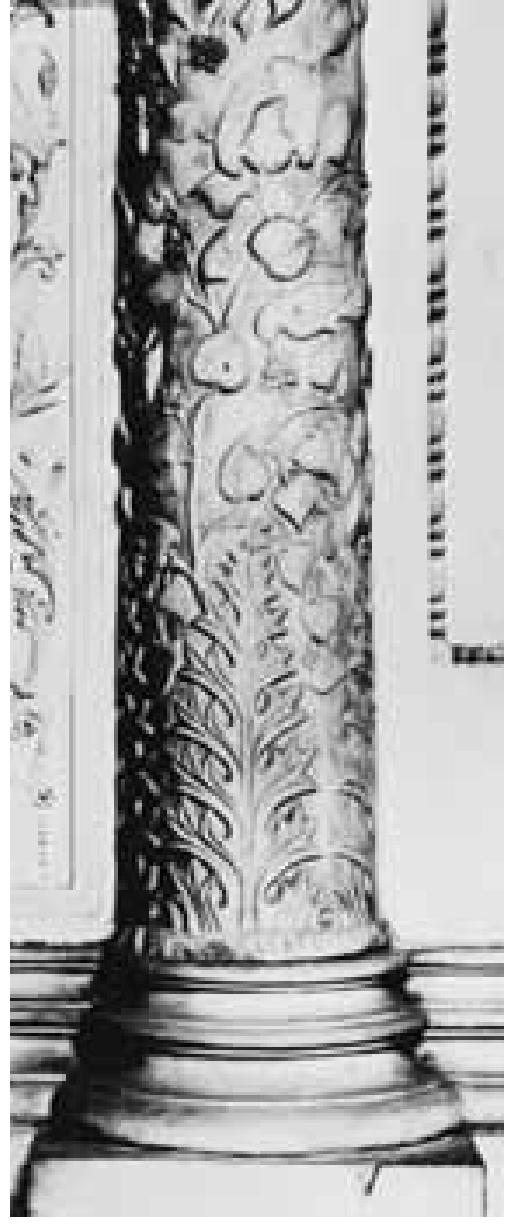

2 Roma, S. Clemente: parte inferiore d'una delle due colonnine del ciborio del tempo di papa Ormisda (514-523), reimpiegate nel monumento funebre del cardinal Venerio di Recanati

\footnotetext{
${ }^{18}$ G. Kapitän, Elementi architettonici per una basilica dal relitto navale del VI secolo di Marzamemi (Siracusa), in: XXVII Corso di cultura sull'arte ravennate e bizantina, Ravenna 1980 (1980) 71-136.

19 Deichmann (nota 2:1989) 321. 322; Sodini (nota 13) 167-168; C. Barsanti, L’esportazione di marmi del Proconneso nelle regioni pontiche durante il IV-VI secolo, RIA 12, 1989 (1990) 107-108; C. Barsanti - A. Guiglia Guidobaldi in: Guidobaldi - Barsanti - Guiglia Guidobaldi (nota 3) 258; Sodini (nota 2:1984) 312; Melucco Vaccaro (nota 3:2001) 398 (il suo arredo marmoreo »documenta anche il livello di finitura assicurato in partenza nei luoghi di origine«); Paroli (nota 14) 132.

${ }^{20}$ Kapitän (nota 18) fig. 6.

${ }^{21}$ Vd. pure Pensabene (nota 13) 348.

${ }^{22}$ Kapitän (nota 18) figg. 4-5.

${ }^{23}$ Vd. J. Kramer, Attische Säulenbasen des 5. und 6. Jahrhunderts n. Chr. und ihre Rohform, BJb 170, 1970, 271-278; F. W. Deich-

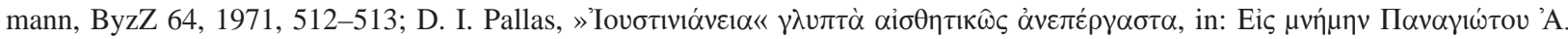
Mıх $\lambda \hat{\eta}$ (1972) 420-441 tavv. 33-38; J. P. Sodini in: Sodini - Kolokotsas (nota 2) 32-34; Barsanti (nota 19) $203-204$ figg. 164-165; E. Russo in: Pillinger (nota 11) 43-44.

${ }^{24}$ E. Russo in: Pillinger (nota 11) 43-44 figg. 42-43 (con la bibl. precedente).

${ }^{25}$ L. Budde, St. Pantaleon von Aphrodisias in Kilikien (1987) 22 tavv. 28-31. 42. 43. 52. 72. 93. 96. 97.

${ }^{26}$ Pallas (nota 23) 420-441.

27 J. P. Sodini in: Sodini - Kolokotsas (nota 2) 34. Né vale l’altra sua supposizione circa il loro invio allo stato di abbozzo a motivo dell'intensa attività edilizia all'epoca e della produzione in serie delle cave: se il lavoro valeva per colonne e capitelli, etc., perché non sarebbe dovuto valere per le basi?

${ }^{28}$ G. Kapitän, The Church Wreck off Marzamemi, Archaeology 22, 1969, 122.
} 
ne e i capitelli lavorati a giorno fossero tutti frutto d'un'importazione dalle officine di Costantinopoli già completamente rifiniti. Tale convinzione era suffragata dal fatto, già notato da F. W. Deichmann, che capitelli lavorati a traforo presenti nella basilica eufrasiana di Parenzo ${ }^{29}$, a S. Vitale ${ }^{30}$ e a S. Apollinare Nuovo ${ }^{31}$ di Ravenna mostrano il tondo destinato al monogramma, da realizzare pur esso a traforo, o non lavorato oppure lavorato con tecnica non a traforo ${ }^{32}$. Ma già allora facevo un'eccezione: i capitelli del ciborio e le transenne dell'altare che il presbitero Mercurio fece erigere in S. Clemente a Roma al tempo di papa Ormisda (514-523) ${ }^{33}$. Allora però non ero in grado di specificare in quale grado di finitezza tali prodotti fossero giunti a Roma. Ma - a parte quel che nel frattempo era emerso a proposito delle transenne e dei capitelli della Palestina $^{34}$ - nei primi anni '90 l'analisi isotopica di uno dei due capitelli superstiti del ciborio di S. Clemente - oggi reimpiegato con l'altro e con le due colonnine superstiti nel monumento funebre del cardinal Venerio di Recanati $(\dagger$ 1479) nella navata sinistra - ha superato nella fattispecie ogni più rosea previsione, dato che il marmo è risultato essere non proconnesio, bensì lunense, di Carrara ${ }^{35}$. Dello stesso marmo sono anche l'altro capitellino ${ }^{36}$ e le due colonnine $\mathrm{e}^{37}$ : mentre però queste ultime son opere classiche, rilavorate nella parte inferiore in occasione del reimpiego nel ciborio (fig. 2$)^{38}$, il secondo capitellino è stato come il primo eseguito completamente a Roma al tempo di papa Ormisda dalle maestranze costantinopolitane ${ }^{39}$. Sono certo della mia affermazione, dato che si tratta di prodotti con le spiccate caratteristiche della scultura coeva della capitale, e frutto degli artefici del più alto livello.

Ma cosa comporta la presenza a Roma di tali maestranze? Prima di procedere desidero richiamare l'attenzione sul fatto che - per una corretta valutazione e un'adeguata soluzione - entra in gioco a questo punto un elemento imprescindibile, vale a dire il criterio dell’interazione uomo-ambiente, cioè della permeabilità delle maestranze, della loro reattività agli stimoli del contesto in cui si trovano a operare, con tutte le tradizioni di

\footnotetext{
${ }^{29}$ F. W. Deichmann, Studien zur Architektur Konstantinopels im 5. und 6. Jahrhundert nach Christus (1956) 52 nota 173; idem, Die Expansion der Architektur Konstantinopels im fünften und sechsten Jahrhundert (1956), in: idem, Rom, Ravenna, Konstantinopel, Naher Osten. Gesammelte Studien zur spätantiken Architektur, Kunst und Geschichte (1982) 628; idem (nota 2:1976) 111. 227; Russo (nota 9:1991) nn. 19. 30.

${ }^{30}$ Deichmann (nota 29:1956) 52 nota 173; Deichmann (nota 29:1982) 628; Deichmann (nota 2:1976) 99, dis. 24; F. W. Deichmann, Ravenna. Hauptstadt des spätantiken Abendlandes III² (1969) tav. 307; Russo (nota 9:1991) 47-49. 61. 62; Aa. Vv., La basilica di San Vitale a Ravenna II (1997) figg. 509-516.

${ }^{31}$ Deichmann (nota 2:1969) 68 fig. 49; Deichmann (nota 2:1974) 139; Deichmann (nota 2:1989) 292; Russo (nota 9:1991) 49-50.

32 Sul problema vd. ora Russo (nota 9:1991) 48-50. 61-62.

33 Russo (nota 1) 10-11. 14 (con la bibl. precedente).

${ }^{34}$ Per le transenne vd. Russo (nota 7) 153-157 figg. 31-34; 164-165. 171 figg. 45-46 a sinistra; 175 fig. 47; 179-180 fig. 49, 3; 185. 193. 199 fig. 61; 201 fig. 63; 206-208 figg. 68-69; 218-220 fig. 77; 241-242. E successivamente: G. Foerster, Decorated Marble Chancel Screens in Sixth Century Synagogues in Palestine and their Relation to Christian Art and Architecture, in: Actes du XI ${ }^{e}$ Congrès international d'archéologie chrétienne (Lyon - Aosta, 21-28 septembre 1986) (1989) 1811 figg. 1-5; Aa. Vv., Pella of the Decapolis II (1989) 122-127 tav. 20A-C; Aa. Vv., Pella in Jordan, 2 (1992) 158 tav. 104a e b; A. Acconci in: M. Piccirillo - E. Alliata, Mount Nebo. New Archaeological Excavations 1967-1997 (1998) 527 n. 151; Ch. Kanellopoulos R. Schick in: Z. T. Fiema et al., The Petra Church (2001) 199-200 figg. 15-17; J. P. Sodini, La sculpture architecturale des églises de Jordanie, in: Les églises de Jordanie et leurs mosaïques. Actes de la journée d’études, Lyon, 22 février 1989 (2003) 139 figg. 43-44; E. Mazar, The Temple Mount Excavations in Jerusalem 1968-1978, II: The Byzantine and Early Islamic Periods (2003) 39. 41 tav. 1, 6. 7. Per i capitelli vd. Russo (nota 7) 164-165. 187-189. 241 fig. 56; 244-246 fig. 88. E successivamente: J. Wilkinson, Column Capitals in al-Haram al-Sharif (1987) 195-203 nn. 139-145; A. Acconci in: M. Piccirillo - E. Alliata, Umm al-Rasas/Mayfa‘ah I: Gli scavi del complesso di Santo Stefano (1994) 310 n. 65 tav. 27, 1; eadem in: M. Piccirillo - E. Alliata, Mount Nebo. New Archaeological Excavations 1967-1997 (1998) 511-513 n. 121 tav. 5, 3; Sodini op. cit. 129 fig. 19; Mazar op. cit. 71 foto I, 85.

${ }^{35}$ F. Guidobaldi in: Guidobaldi - Barsanti - Guiglia Guidobaldi (nota 3) 33.

${ }^{36}$ Ibidem 49.

${ }^{37}$ Ibidem 17.

${ }^{38}$ Ibidem 16-31 figg. 1. 6-16. 32.

${ }^{39}$ Ibidem 32-66 figg. 38-41. 60 (»artefici bizantini di alto livello« presenti a Roma, forse al tempo di papa Ormisda, comunque prima del 533); Melucco Vaccaro (nota 3:1999) 97 (»copie urbane« [!] di »una produzione di serie costantinopolitana«); Sodini (nota 13) 433-434 fig. 18 (prova della presenza a Roma di »ateliers constantinopolitains ou très bien formés à leurs techniques« all'epoca di papa Ormisda); Guiglia Guidobaldi (nota 16) 267 fig. 4 (lavorati »a Roma per opera di artefici costantinopolitani« »al tempo di papa Ormisda e dei suoi successori«); A. Guiglia Guidobaldi in: Ecclesiae Urbis (nota 6) 1482 (»maestranze verosimilmente costantinopolitane« del secondo o terzo decennio del VI sec.).
} 
carattere culturale e storico che tale contesto comporta ${ }^{40}$. Perché noi siamo pronti ad ammetter che Piet Mondrian rispetto alle opere eseguite in Europa (e cito soltanto la composizione B »en bleu, jaune et blanc« del 1936 conservata nel Kunstmuseum di Basilea ${ }^{41}$ ) ha reagito in maniera inequivocabile all'ambiente, nel suo astrattismo, negli anni di vita in America (e mi limito a ricordare il »Broadway BoogieWoogie« del 1942/43, nel Museum of Modern Art di New York $^{42}$ ), e non siamo disposti a riconoscer che la grande realtà storica e artistica di Roma non abbia lasciato del tutto indifferenti le maestranze greco-costantinopolitane ch'eran giunte sulle rive del Tevere?

Consideriamo innanzi tutto la decorazione del capitellino a 2 zone di sinistra (fig. 3), che reca la scritta [ser]bus $D($ omi $) n i \dagger$ Mercurius $p($ res $) b(y t e r) s(a n) c(t a) e$ ecclesiae catholicae off(erit $)^{43}$, e capiremo súbito anche il peso della committenza, quel processo che kitzingerianamente possiam definire eterodiretto $0^{44}$ e che impone di trovar una soluzione diversa dallo spazio circolare di un monogramma per una

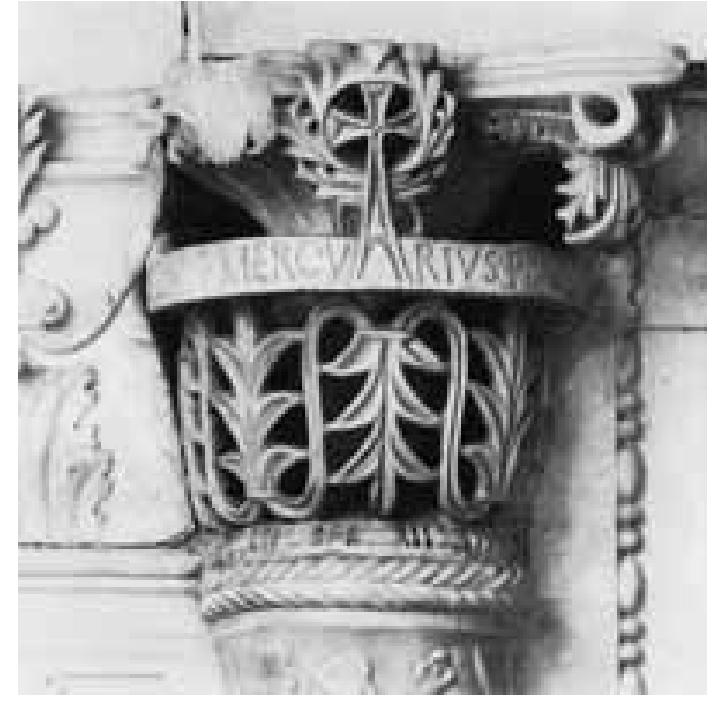

3 Roma, S. Clemente: capitellino a 2 zone reimpiegato sulla colonnina di sinistra del monumento funebre del cardinal Venerio di Recanati scritta dedicatoria che si sviluppa longitudinalmente ma sempre secondo la circolarità del manufatto. Di qui l'unicità del pezzo $^{45}$, con l'evidenza della fascia orizzontale piena e piatta e il forte chiaroscuro, dove il vuoto, il nero, prevale sul bianco, sul pieno della parte lavorata virtuosisticamente a traforo; è pure un unicum nella zona superiore il nesso tra il classico abaco formato da ovolo, tavoletta e cavetto - ma con l'inserzione d'una sorta di voluta che fà tutt'uno con il cavetto - e le foglie angolari a mo' di elici ${ }^{46}$. Unico è anche, con quel risalto, il motivo vegetale a 7 elementi disposti alternati secondo l'andamento del nastro a 2 bande, che compare pure nella zona superiore della campana dei quattro capitelli compositi nel nartece di S. Giovanni di

${ }^{40}$ È un criterio su cui sono solito insistere, in presenza dell'opera di maestranze venute dall'esterno: vd. da ultimo E. Russo, L'architettura di Ravenna paleocristiana (2003) 56, e idem, La scultura di S. Polieucto e la presenza della Persia nella cultura artistica di Costantinopoli nel VI secolo, in: La Persia e Bisanzio. Atti del Convegno internazionale di studi, Roma, 14-18 ottobre 2002 (2004) 764-765.

41 A. Busignani, Mondrian (1968) 23-24. 38 fig. 72; J. M. Joosten, Piet Mondrian, II. Catalogue Raisonné of the Work of 1911-1944 (1998) 383-384, B 263. Ma si potrebbero ugualmente ricordare, del 1936, le composizioni A (Los Angeles, County Museum of Art, inv. 63.14) (Aa. Vv., Piet Mondrian. 1872-1944 [1994] 265 n. 144) e C (Zurigo, Collezione privata) (ibidem $267-268$ n. 146).

42 Busignani (nota 41) 24. 38 fig. 77; Piet Mondrian (nota 41) 292-293 n. 165; Joosten (nota 41) 92. 418-419, B 323. Ma vale la pena di citare almeno »New York City« (Parigi, Musée national d’art moderne) del 1941-42 (Busignani [nota 41] 24. 38 fig. 76; Piet Mondrian [nota 41] 290-291 n. 164).

43 È questa la lettura proposta da F. Guidobaldi in: Guidobaldi - Barsanti - Guiglia Guidobaldi (nota 3) 34-36, in séguito a un'ispezione diretta e ravvicinata; sciolgo però OFF con off(erit), e non con off(ert) come propone Guidobaldi, in base alla scritta sulla copertina dell'evangeliario di Monza.

${ }^{44}$ E. Kitzinger, On the Interpretation of Stylistic Changes in Late Antique Art, Bucknell Review 15, 3, 1967, 1-10, ristampato in: idem, The Art of Byzantium and the Medieval West (1976) 32-41 (per i concetti di »other-directed« e »inner-directed«).

${ }^{45}$ F. Guidobaldi s'è reso conto nella sua lunga analisi dell'eccezionalità del pezzo, ma non è riuscito a coglier il motivo (il processo eterodiretto) che ha generato tale eccezionalità.

${ }^{46}$ F. Guidobaldi in: Guidobaldi - Barsanti - Guiglia Guidobaldi (nota 3) 45-46 figg. 43. 51, propone il confronto con un capitello reimpiegato nel campanile di Pomposa e con un altro del Museo archeologico d’Istanbul, proveniente dal Hebdomon. Ma, a parte la struttura e la qualità, nel primo caso sopra le foglie abbiamo i caulicoli con le volute, nel secondo caso solo foglie angolari schematizzate con volute (il capitello dal Hebdomon - su cui vd. Th. Zollt, Kapitellplastik Konstantinopels vom 4 . bis 6. Jahrhundert n. Chr. [1994] 222-223 n. 638 tav. 45 - è interessante per noi dato il rapporto zona inferiore - zona superiore assai simile al capitello di S. Clemente). Più convincente appare invece la proposta di veder un legame tra la soluzione adottata a S. Clemente e quella presente nei capitelli, però corinzii, di Gortina nell'isola di Creta, di Nea Anchialos, di S. Marco di Venezia (F. Gudiobaldi in: Guidobaldi - Barsanti - Guiglia Guidobaldi [nota 3] 46-47 figg. 57. 59): soluzioni però simili, non identiche, prova dell’origine e della cultura delle maestranze, ma dell'assenza di serialità nel lavoro di S. Clemente. 


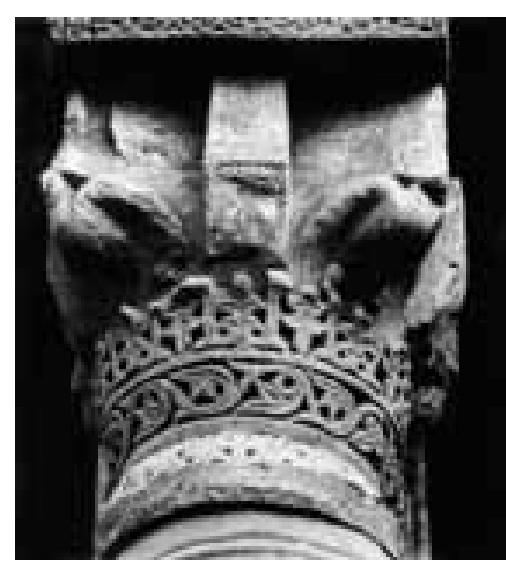

4 Il Cairo, moschea di Șāliḥ Ṭalā‘i: capitello a 2 zone reimpiegato

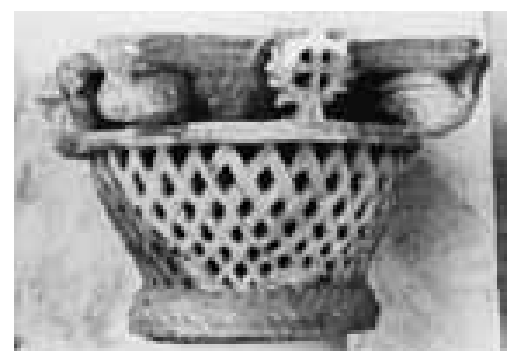

5 Beirut, Museo: capitello a 2 zone, da Tiro
Studios a Costantinopoli ${ }^{47}$ intorno alla metà del V sec., e, nei capitelli del nostro tipo, soltanto in un esemplare della moschea di Șālị̣ Țalā‘i al Cairo (fig. 4$)^{48}$ - ma a 5 elementi - e in due capitelli del Museo archeologico d'Istanbul ${ }^{49}$ - ma a 9 elementi; invece il motivo è più comune nelle cornici, come fanno fede i frammenti reimpiegati in uno dei piloni di rinforzo davanti alla facciata occidentale di S. Sofia di Costantinopoli ${ }^{50}$ e quelli conservati nel Museo archeologico d'Istanbul ${ }^{51}$. L'acrobatica croce latina che fuoresce decisamente con l'asta verticale, quasi un inizio di coppia di lemnisci in basso, non costituisce un unicum nei capitelli a 2 zone, sebbene sia fenomeno raro: fuoresce decisamente anche in circa otto esemplari della basilica doppia di Aliki nell'isola di Thasos ${ }^{52}$, in due capitelli reimpiegati ai lati dell'ingresso sul lato est di S. Sofia a Costantinopoli $^{53}$, in uno del Museo di Antalya ${ }^{54}$, in uno del Museo di Beirut (da Tiro) (fig. 5) ${ }^{55}$, in uno del Museo copto del Cairo (proveniente da alFuștāt $)^{56}$; ma in nessuno degli esempi citati il motivo ha lo slancio e l'eleganza del capitello di S. Clemente. A parte la fascia con l'iscrizione (che però provoca, come in una reazione a catena, le varie peculiarità del pezzo), tutto il resto, a cominciare dalla scelta del tipo a 2 zone, è frutto d'un'opera intradiretta delle maestranze della capitale sul Bosforo.

Considerazioni analoghe possono svilupparsi per l'altro capitello a 2 zone superstite (fig. 6), giacché un paniere dalle maglie tanto larghe - prova di supremo virtuosismo tecnico - e così sviluppato in altezza, in modo da costringer le colombe angolari della zona superiore a una posizione orizzontale, è anch'esso praticamente senza riscontri. L'altezza del paniere in rapporto alla zona superiore è infatti maggiore rispetto a quella verificabile nel capitello del Museo archeologico di Pola, proveniente dal battistero della cattedrale ${ }^{57}$, e a mia conoscenza è raggiunta soltanto dal

47 Zollt (nota 46) 209 n. 599 tav. 44; J. Kramer, Bemerkungen zu den Methoden der Klassifizierung und Datierung frühchristlicher oströmischer Kapitelle, in: Spätantike und byzantinische Bauskulptur. Beiträge eines Symposions, Mainz, 18.-20. Februar 1994 (1998) 56 tav. 14, 26-29. Buona riproduzione in: Th. F. Mathews, The Byzantine Churches of Istanbul. A Photographic Survey (1976) fig. 15-20.

${ }^{48}$ R. Kautzsch, Kapitellstudien (1936) 160-161 n. 511; E. Kitzinger, The Horse and Lion Tapestry at Dumbarton Oaks, DOP 3, 1946, 71 n. 98; K. A. C. Creswell, The Muslim Architecture of Egypt I (1952) tav. 104A; A. Guiglia Guidobaldi, Scultura costantinopolitana del VI secolo: i capitelli reimpiegati nella medresa della moschea di Davut Pasha, in: Milion I (1988) 233 tav. 4, 2; H. G. Severin, Konstantinopler Bauskulptur und die Provinz Ägypten, in: Spätantike und byzantinische Bauskulptur (nota 47) 97 tav. 29, 4; idem, Zur Skulptur und Malerei der spätantiken und frühmittelalterlichen Zeit in Ägypten, in: Aa. Vv., Ägypten in spätantik-christlicher Zeit. Einführung in die koptische Kultur (1998) 318 fig. 9; Sodini (nota 13) 433 fig. 19; K. Krumeich, Spätantike Bauskulptur aus Oxyrhynchos I (2003) 95 fig. 36.

${ }^{49}$ C. Barsanti in: Sodini - Barsanti - Guiglia Guidobaldi (nota 2) 326-327 fig. 18.

${ }^{50}$ C. Barsanti - A. Guiglia Guidobaldi, Premessa ad un catalogo della scultura della Santa Sofia di Costantinopoli, in: Bisanzio e l'Occidente: arte, archeologia, storia (1996) 84 fig. 7.

${ }^{51}$ N. Firatll, La sculpture byzantine figurée au Musée archéologique d'Istanbul (1990) nn. 263. 378.

52 J. P. Sodini in: Sodini - Kolokotsas (nota 2) 35-37 tav. 15f fig. 38; 37 tav. $16 f$ fig. 40 (due esemplari); 37 tav. 16g (due esemplari); 37 tav. $16 \mathrm{f}$ e g (almeno tre esemplari).

${ }^{53}$ Kautzsch (nota 48) 163-164 n. 522 tav. 32; Kitzinger (nota 48) 69 n. 77 fig. 107; Zollt (nota 46) 226 nn. 647-648; J. Kramer, Frühchristliche Kapitelle mit Taubenfiguren an San Marco in Venedig und ihre Verwendung, in: Vom Orient bis an den Rhein. Begegnungen mit der christlichen Archäologie (1997) 109. 117 figg. 6-7; idem (nota 47) 46 tav. 10, 2 e 4.

54 J. P. Sodini in: Sodini - Kolokotsas (nota 2) 40 tav. 17c; Kramer (nota 53) 109. 120 fig. 9; U. Peschlow, Tradition und Innovation: Kapitellskulptur in Lykien, in: Spätantike und byzantinische Bauskulptur (nota 47) 70 tav. 20, 13.

55 J. P. Sodini in: Sodini - Kolokotsas (nota 2) 40 tav. 17d.

${ }^{56}$ Kautzsch (nota 48) 164 n. 523 tav. 32; Kitzinger (nota 48) 69 n. 81 fig. 111; H. G. Severin in: Spätantike und byzantinische Bauskulptur (nota 47) 98 tav. 31, 13; Krumeich (nota 48) 65-66 fig. 19.

${ }^{57}$ Kitzinger (nota 48) 69 n. 84 fig. 114; B. Marušić, Istrien im Frühmittelalter ${ }^{3}$ (1969) 14 tav. 11, 2; idem in: Museo archeologico d’Istria, Pola. Guida (1979) 20 fig. 9. 


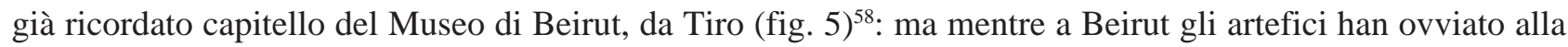
ristrettezza dello spazio ponendo la testa delle colombe tangente al bordo laterale dell'abaco, e grazie a tale accorgimento han potuto delineare un profilo più naturale all'atteggiamento dei quattro uccelli, a S. Clemente l'ampiezza dell'abaco in confronto al paniere ha obbligato gli esecutori a una postura orizzontale altrimenti ignota, dato che pure la testa delle colombe risulta sottostante al cavetto. Il livello qualitativo è il medesimo del primo capitello, poiché medesima è la maestranza, per due pezzi del medesimo ciborio ${ }^{59}$ : è evidente altresì che il paniere tanto sviluppato in altezza è scelta deliberata per uniformarsi all'ampiezza della zona inferiore del primo capitello.

Ma v'è di più. Il peso dell'ambiente culturale romano si avverte nettissimo - anche se ciò non è stato ancora notato - nella scelta da parte di maestranze abituate a lavorare marmi ex novo, di reimpiegare, secondo la pratica romana, colonne classiche, rilavorandone parzialmente soltanto la zona inferiore (fig. 2), e in più applicando, nell'impiego di differenti decorazioni nei capitelli a 2 zone, quell'estetica della varietas su cui abbiam riflettuto $\mathrm{io}^{60} \mathrm{e}$ il collega $\mathrm{B}$. Brenk $\mathrm{k}^{61}$, varietas che con il reimpiego connotava la decorazione scultorea delle chiese di Roma dall'età costantiniana ma a Costantinopoli era testé comparsa con il S. Polieucto di Anicia Iuliana ${ }^{62}$. A motivo di tale estetica, e tenendo conto del criterio della simmetria delle coppie ${ }^{63}$ lar- $^{2}$ gamente applicato nella varietas, io credo che il capitello con l'iscrizione fosse ubicato con altro identico pure con scritta - nella parte anteriore del ciborio, mentre quello con il paniere assieme a un altro gemello fosse posizionato sulle colonnine posteriori del manufatto.

E non soltanto questo. Giacché nella ricostruzione medievale del recinto di S. Clemente son state reimpiegate, ruotate di $90^{\circ}$ rispetto alla loro originaria posizione ${ }^{64}$, tre virtuosistiche transenne coeve al ciborio di Mercurio $^{65}$ e facenti parte dell'altare (figg. 7. 8. 9) ${ }^{66}$. Le tre transenne son connotate da un motivo a stuoia, che non trova riscontro nella produzione di transenne di Costantinopoli o delle maestranze greco-costantino-

\footnotetext{
58 J. P. Sodini in: Sodini - Kolokotsas (nota 2) 40 tav. 17d ed e.

59 Non convincono infatti le ondeggianti argomentazioni di F. Guidobaldi in: Guidobaldi - Barsanti - Guiglia Guidobaldi (nota 3) 59-66, volte a sostenere la non appartenenza dei capitelli a un ciborio, e comunque in funzione non paritaria.

${ }^{60}$ Russo (nota 9:1991) passim; E. Russo in: Pillinger (nota 11) 30-34. 39-47 figg. 9-11. 28-31. 36-58; idem in: Aurea Roma (nota 6) 191-194; idem in: La Persia e Bisanzio (nota 40) 764-765. 815-817; idem, Il pulvino sopra il capitello a cesto, Bizantinistica
} n. s. 7 (2005, in corso di stampa).

${ }^{61}$ B. Brenk, Zum Baukonzept von Hagios Demetrios in Thessaloniki, Boreas 17, 1994, 27-38; idem, Spolien und ihre Wirkung auf die Ästhetik der varietas. Zum Problem alternierenden Kapitelltypen, in: J. Poeschke (ed.), Antike Spolien in der Architektur des Mittelalters und der Renaissance (1996) 49-80.

62 E. Russo in: La Persia e Bisanzio (nota 40) passim 737-826; idem (nota 60:in corso di stampa).

${ }^{63}$ F. W. Deichmann, Säule und Ordnung in der frühchristlichen Architektur, RM 55, 1940, 119-130; idem, Die Spolien in der spätantiken Architektur, SBMünchen (1975) 9. 91-94.

${ }^{64}$ Così già R. Kautzsch, Die römische Schmuckkunst in Stein vom 6. bis zum 10. Jahrhundert, Römisches Jahrbuch für Kunstgeschichte 3, 1939, 50 fig. 81.

${ }_{65}$ Russo (nota 1) 10-11 (con la bibl. precedente); C. Barsanti in: Guidobaldi - Barsanti - Guiglia Guidobaldi (nota 3) 86. 87-88. 93. 186-193 figg. 85-86. 91-92. 118-119. Per la Barsanti, la lastra laterale, ibidem 86. 192-193 figg. 85-86 »va distaccata cronologicamente« dalle altre due, »e va dunque considerata probabilmente come un'aggiunta o una sostituzione in quel non meglio individuabile arredo liturgico del quale facevano parte le altre due transenne«, ma »Difficile è certo precisare l'epoca in cui tale transenna fu eseguita«. La Barsanti è seguíta dalla Paroli (nota 14) 135 fig. 102, la quale dichiara che la transenna »imita« le altre due "protobizantine«, assegnandole l'VIII secolo. Ho riesaminato attentamente le tre transenne e son giunto di nuovo alla conclusione che la differenza non è cronologica, ma soltanto qualitativa. La mano è abilissima, ma non quanto quella delle altre due transenne: tutto però spinge a considerare la transenna appartenente alla stessa temperie culturale e tecnica e allo stesso complesso: il campo a traforo $(54 \times 75$, di contro a $54 \times 80,5$ a sinistra e a $54 \times 81$ a destra), lo spessore della parte a traforo (1,5 circa, di contro a 2,5 e a 2,5 circa), il motivo dell'incorniciatura originariamente in alto (47,5 oggi, in origine 9, di contro a 7,5 e a 7,5), praticamente identico a quello delle altre due, mentre il motivo in origine ai lati (11 in alto, 13,5 in basso) è altrettanto complesso e ben condotto, le stesse dimensioni complessive $(74 \times 115$, di contro a $83 \times 114$ e a $81 \times 114)$. Certo si può discutere del motivo per cui il nastro è a 3 e non a 2 solchi e del perché i fori siano romboidali e non circolari: ma una datazione posteriore al VI secolo è improponibile, e soprattutto la mano è come negli altri due pezzi quella d'un artefice costantinopolitano. Volendo andare a caccia di 'errori', si può notare la differenza dell'andamento del nastro a sinistra in alto rispetto a destra in basso, ma la trama è regolarissima e condotta con maestria. Io poi credo pure che l'adozione del nastro a 4 bande e non a 3 sia una conseguenza dell'ampliamento - e su una lastra di spessore inferiore alle altre due - della trama con conseguenti maggiori dimensioni dei fori, per evitare che in séguito all'allargamento la transenna rischiasse maggiormente di spezzarsi.

${ }^{66}$ Escludendo l'altare, C. Barsanti in: Guidobaldi - Barsanti - Guiglia Guidobaldi (nota 3) 186-187. 192, non riesce infatti a trovare una spiegazione per le transenne, e F. Guidobaldi, dopo aver a più riprese contestato l'appartenenza a un altare, è alla fine 


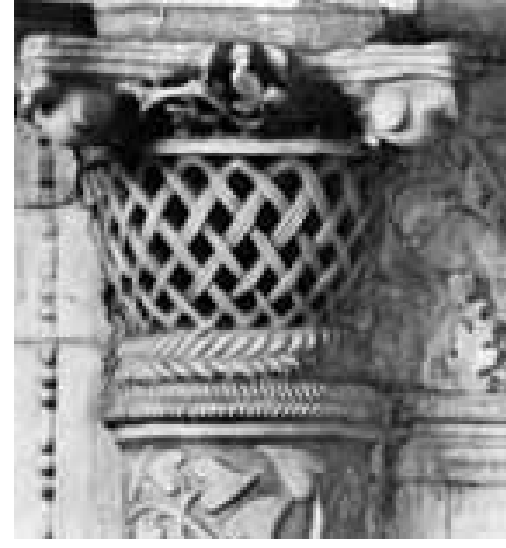

6 Roma, S. Clemente: capitellino a 2 zone reimpiegato sulla colonnina di destra del monumento Recanati funebre del cardinal Venerio di

politane, dato che un piccolo frammento di pluteo (dunque non transenna) rinvenuto nei pressi della basilica della Natività a Betlemme ${ }^{67}$ - già da me attribuito a maestranze greco-costantinopolitane operanti in Palestina nella seconda metà del VI secolo - e richiamato a confronto da C. Barsanti mostra nell'esiguo resto del campo interno non un motivo a stuoia come crede la studiosa, bensì chiaramente »un intreccio a matassa con nastro a tre bande ${ }^{68}$. Invece il motivo a stuoia a ornare con la sua densa trama il campo interno delle lastre compare in una serie di plutei, lavorati in origine col motivo a cancello - tipicamente romano - nell'altra faccia, di S. Agnese f. l. m. (figg. 10. 11) ${ }^{69}$. Ora i pezzi di S. Agnese son da riferire a mio parere alla basilica costantiniana dedicata alla martire ${ }^{70}$ : e ciò è un fatto d'estrema importanza anche per le considerazioni future sulle recinzioni liturgiche a Roma ${ }^{71}$; come allo stesso edificio vanno attribuiti due coevi capitelli a paniere oggi nell'attiguo Protettorato di S. Giuseppe (figg. 12. 13) ${ }^{72}$. Orbene io credo che tanto il capitello a paniere del ciborio del tempo di papa Ormisda quanto le tre coeve transenne dell'altare col motivo a stuoia posson esser fatti risalire all'osservazione e alla deliberata ripresa delle decorazioni della basilica costantiniana di S. Agnese f. l. m. da parte delle maestranze costantinopolitane presenti a Roma ${ }^{73}$. L'accertata attività a Roma di artefici costantinopolitani, traguardata col criterio dell'interazione uomo-ambiente, all'epoca di Ormisda ci ha permesso anche di chiarire un aspetto assai significativo (si pensi soltanto alla collocazione cronologica dei capitelli a paniere oggi nel Protettorato di S. Giuseppe) e di porre ulteriori quesiti (come la funzione

costretto ad ammetter che »è più semplice, anche se non provato, attribuire all'altare menzionato nell'iscrizione le transenne« (F. Guidobaldi in: Materali e tecniche [nota 17] 178 fig. 7).

${ }^{67}$ Per cui vd. B. Bagatti, Gli antichi edifici sacri di Betlemme (1952) 50. 281 foto 29, 3; Russo (nota 7) 165 fig. 40 al centro; C. Barsanti in: Guidobaldi - Barsanti - Guiglia Guidobaldi (nota 3) 188 fig. 286.

${ }^{68}$ Russo (nota 7) 165.

${ }^{69}$ U. Broccoli, La diocesi di Roma V, Corpus della scultura altomedievale VII 5 (1981) 157-164 nn. 113-118 tavv. 27-29 (definite erroneamente transenne); C. Barsanti in: Guidobaldi - Barsanti - Guiglia Guidobaldi (nota 3) 188-190 figg. $287-289$ (plutei e non transenne). Il Broccoli fà riferimento, ma imprecisamente, all’»ambiente ravennate«, e data i pezzi »Intorno alla metà del secolo VI«; la Barsanti invece giustamente nota le differenze formali con le transenne di S. Clemente, e suggerisce »di considerare con estrema cautela una datazione posteriore agli inizi del IV secolo«.

70 Sulla basilica circiforme di S. Agnese vd. da ultimo D. Esposito - P. Venturini, La basilica cimiteriale di S. Agnese fuori le mura a Roma: nuove osservazioni e ipotesi, Quaderni dell'Istituto di Storia dell'Architettura n. s. 23, 1993, 3-16; E. Giuliani in: E. Giuliani - C. Pavolini, La 'biblioteca' di Agapito e la basilica di S. Agnese, in: The Transformations of Vrbs Roma in Late Antiquity (1999) 99-103; P. M. Barbini - F. Severini, Risultati archeologici del nuovo saggio di scavo 1999 nella basilica cimiteriale di S. Agnese, in: Ecclesiae Urbis (nota 6) 757-770; C. Pavolini, La basilica costantiniana di S. Agnese. I risultati delle indagini e dei restauri per il Giubileo del 2000, in: Ecclesiae Urbis (nota 6) 1203-1224; D. Esposito, La basilica cimiteriale di S. Agnese f. l. m.: interpretazione e utilizzo dei ruderi dal XV al XIX secolo, in: ibidem 1391-1410; M. Magnani Cianetti, Il muro perimetrale della basilica costantiniana di S. Agnese alla luce dei recenti restauri, in: ibidem 1411-1428; M. Magnani Cianetti - C. Pavolini (edd.), La basilica costantiniana di Sant’Agnese. Lavori archeologici e di restauro (2004).

${ }^{71}$ Per U. Broccoli i pezzi vanno riferiti »alla basilica major, o addirittura, a quel qualcosa di non chiaramente identificato che sorse sul corpo della santa (Broccoli [nota 69] 160-161. 193): è impossibile però che lastre di grandi dimensioni fossero contenute in »una piccola basilica, forse totalmente ipogea«.

72 Broccoli (nota 69) 191-193 nn. 140-141 tav. 38 (non esclude »una comunanza di botteghe, oltre che una datazione contemporanea« ai plutei; VI sec.; vd. pure nota precedente); Pensabene (nota 13) 293. 350.828 nota 54 (»tardo V secolo«, »con influssi siriani«); A. Guiglia Gudiobaldi, Precisazioni su alcuni capitelli a canestro di ambito romano, in: Quaeritur inventus colitur. Miscellanea in onore di P. Umberto M. Fasola (1989) 407-422 figg. 2-3 (marmo lunense; improbabilità di una matrice bizantina, lontani anche da quelli romani del II sec.; »epoca non troppo lontana dall'età imperiale avanzata«).

${ }^{73}$ C. Barsanti in: Guidobaldi - Barsanti - Guiglia Guidobaldi (nota 3) 190, preferisce considerare i plutei di S. Agnese »solo come eventuale fonte d'ispirazione per« le transenne. Ma siccome è tutta orientata, assieme alla Guiglia, a circoscrivere l'attività a Roma delle maestranze costantinopolitane soltanto ai capitellini del ciborio, non dà corso a questo pensiero, e di fatto esclude la »eventuale fonte d'ispirazione«, che ha come conseguenza inevitabile l'esecuzione a Roma da parte di artefici costantinopolitani delle transenne col motivo a stuoia in marmo del Proconneso. Ciò è comprovato dalle conclusioni: »Il fatto che esse siano di marmo proconnesio e non di Carrara come i capitelli lascia comunque aperto il campo ad altre soluzioni« (C. Barsanti - A. Guiglia Guidobaldi in: Guidobaldi - Barsanti - Guiglia Guidobaldi [nota 3] 255). 
di lastre e capitelli nell'edificio) per l'articolazione e le vicende della basilica del IV secolo. Segnalo anche un piccolo frammento di pluteo pure coevo agli altri di S. Agnese e con l'identico motivo a stuoia nella parte anteriore e a cancello in quella posteriore ${ }^{74}$, oggi nel Museo Pio Cristiano Vaticano ${ }^{75}$, inv. 31334 (fig. 14), dove si conserva anche un altro piccolo frammento, inv. 50478 (fig. 15) ${ }^{76}$, il quale ci dimostra che la produzione era continuata ben oltre il IV secolo.

Dobbiam fare attenzione alle date. I pezzi di S. Clemente con decorazione a traforo, di alto livello, sono del tempo di papa Ormisda (514-523). La lavorazione a giorno vede a Costantinopoli quali prototipi le sculture della già menzionata chiesa di S. Polieucto ${ }^{77}$, giacché tutti i tentativi di collocar anteriormente alla chiesa di Anicia Iuliana le novità rivoluzionarie introdotte invece dall'edificio della capitale sul Bosforo si son rivelati inadeguati o errati ${ }^{78}$. Finora il S. Polieucto è stato ritenuto una ricostruzione realizzata da Anicia Iuliana tra il 524 e il $527^{79}$. Si avrebbe allora un'incongruenza cronologica con le sculture di S. Clemente, anterio-

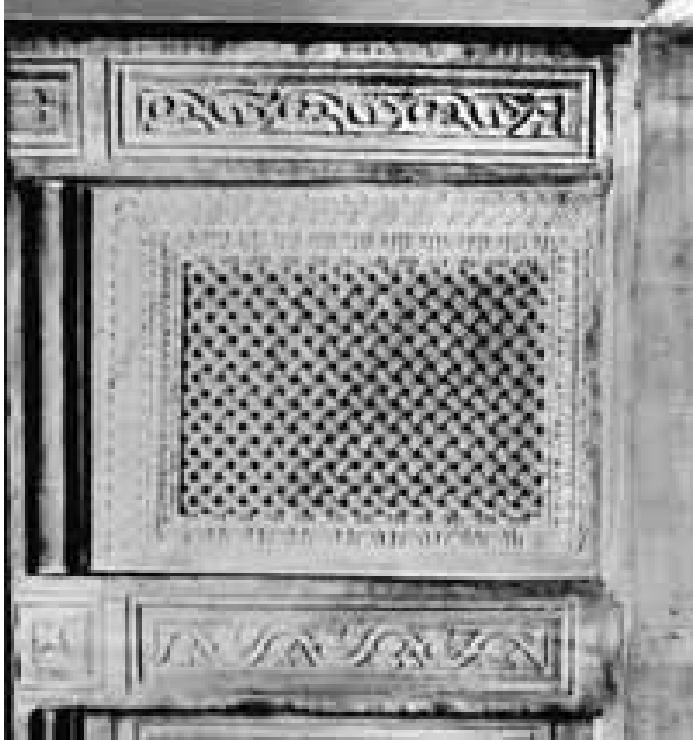

7 Roma, S. Clemente: transenna dell’altare del tempo di papa Ormisda ri al 524. Invece i pezzi del ciborio e dell'altare del presbitero Mercurio offrono una conferma indiretta della datazione brevemente proposta per S. Polieucto da J. Bardill nel $1994^{80}$ e da lui precisata in un libro appena pubblicato in base ai bolli laterizii: secondo lo studioso inglese la chiesa di Anicia Iuliana sarebbe stata ricostruita per la parte sopratterra tra il 517 e il $521^{81}$. Tutto ciò potrebbe portar a datare quasi ad annum il ciborio e l'altare tra il 522 e il 523.

Le tre transenne col motivo a stuoia (figg. 7. 8. 9) son in marmo del Proconneso ${ }^{82}$. Ciò è un fatto che implica l'invio di lastre di marmo allo stato grezzo, assieme alle maestranze, e non soltanto la presenza delle maestranze costantinopolitane ${ }^{83}$. Questo è un punto d'estrema importanza nel momento in cui passiam a esaminare il complesso di plutei e pilastrini di S. Clemente eseguiti, in marmo proconnesio, sotto Giovanni II (533-35) $)^{84}$, il medesimo Mercurio allora divenuto papa, ma legatissimo alla chiesa di cui era stato presbi-

${ }^{74}$ Marmo; cm. 25 h × 32,5 × 6 sp. La fascia è alta cm. 5,5, il kymation cm. 8. Ringrazio cordialmente la dott.ssa L. Vattuone per avermi agevolato nell'esame di questo e del pezzo seguente, e il dott. F. Buranelli per il permesso di pubblicarne le fotografie.

75 O. Marucchi, I monumenti del Museo Cristiano Pio-Lateranense (1910) 12 tav. 8, 2 (detto »transenna« e »d'epoca tarda«). Il frammento è opportunamente richiamato anche da C. Barsanti in: Guidobaldi - Barsanti - Guiglia Guidobaldi (nota 3) 188 nota 320. Nell'»Inventario del Museo Pio-Lateranense o Cristiano nel Palazzo Apostolico del Laterano« il pezzo, con il n. 32 (ora 31334) è detto provenire da Porto, 1866, dono di Alessandro Torlonia, con data d'entrata al Museo 16.V.1868.

${ }^{76}$ Marmo; cm. $20 \mathrm{~h} \times 34 \times 5 \mathrm{sp}$. La decorazione è costituita sulla fronte da un corrimano superiore bombato $(\mathrm{cm}$. $3 \mathrm{~h})$, da una fascia con croce incavata e grandi foglie d'edera contenenti un motivo gigliato (la fascia è h cm. 9,5 compresi i due bordini, cm. 6 all'interno), un kymation e una fila di astragali (cm. 7,5 la loro altezza); nel retro il corrimano bombato (cm. 3,5-4) è seguíto da una fascia di $\mathrm{cm} .9$ e da una sagomatura a gola rovescia e diritta di $\mathrm{cm} .7$.

77 Per cui rimando a E. Russo in: La Persia e Bisanzio (nota 40) 737-826, e Russo (nota 60:in corso di stampa).

${ }^{78}$ Vd. E. Russo in: La Persia e Bisanzio (nota 40) 741-746, e Russo (nota 60:in corso di stampa).

${ }^{79}$ C. Mango - I. Ševčenko, Remains of the Church of St. Polyeuktos at Constantinople, DOP 15, 1961, 244-245; R. M. Harrison, The Church of St. Polyeuktos in Constantinople. An Excavation Report, in: Akten des VII. Internationalen Kongresses für Christliche Archäologie (Trier, 5.-11. September 1965) (1969) 545; idem, Excavations at Saraçhane in Istanbul I (1986) 3-4.

80 J. Bardill, Brickstamps and the Date of St. Polyeuktos, Bulletin of British Byzantine Studies 20, 1994, 67 (datazione entro il 521-22).

${ }^{81}$ J. Bardill, Brickstamps of Constantinople (2004) 24-25. 26. 33. 62-64. 82. 101. 111-116. 125-126. 139-140. 152 tav. 7 figg. 15-16 tavv. 5, 14-16: le fondazioni sotterranee della chiesa possono risalire ad Areobindo, marito di Anicia Iuliana, nel o súbito dopo il 508-09 e la loro costruzione sarebbe cessata nel 511-12, la parte in alzato sarebbe stata costruita da Anicia dopo la morte del marito tra il 517 e il 521; nella chiesa sarebbero stati riadoperati mattoni dell'edificio precedente del V sec.

${ }^{82}$ C. Barsanti - A. Guiglia Guidobaldi in: Guidobaldi - Barsanti - Guiglia Guidobaldi (nota 3) 255.

${ }^{83}$ Come avevo sostenuto già in Russo (nota 1) 10-11.

${ }^{84}$ Russo (nota 1) 11-19 figg. 2-4; Russo (nota 6:1989) 597-610 fig. 1; C. Barsanti - A. Guiglia Guidobaldi in: Guidobaldi - Barsanti - Guiglia Guidobaldi (nota 3) 69-264 figg. 78-210; A. Guiglia Guidobaldi in: Christiana loca II (nota 14) 202-203 fig. a p. 202. 


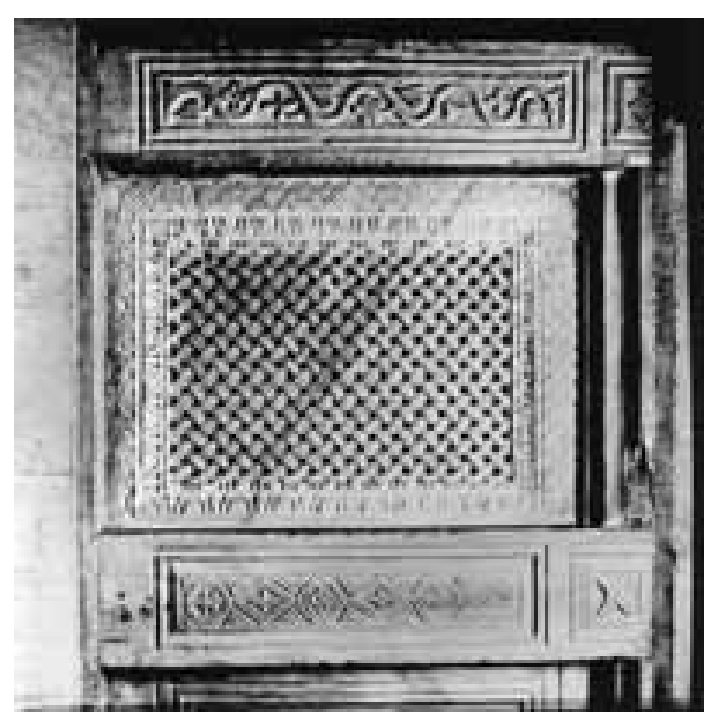

8 Roma, S. Clemente: transenna dell'altare del tempo di papa Ormisda

tero. Infatti oggi gli studiosi - come dicevo più sopra - son d'accordo nel ritenere che, a parte l'episodica presenza di maestranze costantinopolitane a Roma sotto papa Ormisda, per il resto le sculture di S. Clemente sian frutto d'un'importazione di pezzi già lavorati: uno o più carichi, ripartiti súbito prima e al massimo súbito dopo le guerre gotiche che avrebbero coinvolto la scultura architettonica e decorativa delle altre chiese romane dove si conservano opere grecocostantinopolitane. Ma la presenza - in ben cinque dei sei plutei (in realtà con ogni probabilità sette su otto se consideriamo anche i due rilavorati nel medio evo) con il motivo del clipeo o corona lemniscata e affiancata da due croci sostenute da una foglia d'edera ${ }^{85}$ - del monogramma di papa Giovanni II in luogo della canonica croce o del monogramma cristologico a 6 o 8 bracci (fig. 16) è una sostituzione audacissima che soltanto la deliberata volontà del committente (ancor una scelta eterodiretta nell'àmbito d'un processo intradiretto) poteva imporre ${ }^{86}$. È questa una soluzione assai rara nei plutei ${ }^{87}$, e trova riscontro soltanto in lastre di S. Demetrio di Salonicco, per ricordare il magister Gregorio (fig. 17) ${ }^{88}$, della chiesa cruciforme di Salona (con il monogramma del vescovo Onorio II, sicuramente vivo nel 547) ${ }^{89}$, in S. Sofia di Nicea ${ }^{90}$ : tutti pezzi lavorati sul posto, come quelli di S. Clemente. Di contro all'unico pluteo di S. Clemente con monogramma cristologico a 6 bracci entro il clipeo centrale, e per di più non rifinito, le maestranze costantinopolitane presenti a Roma si son dedicate a realizzare attorno al monogramma papale volutamente sempre rilevato o un semplice clipeo, o una corona d'alloro, o una corona con tralcio e foglie

\footnotetext{
${ }^{85}$ Su questo schema diffusissimo vd. E. Russo, Nota su due sarcofagi del VI secolo inediti conservati nella chiesa di S. Agata di Ravenna, Studi romagnoli 19, 1968 (1971) 321-329, con alcuni complementi in Sodini (nota 2:1984) 286, e in R. Farioli, Ravenna, Costantinopoli. Considerazioni sulla scultura del VI secolo, in: XXX Corso di cultura sull'arte ravennate e bizantina: Seminario giustinianeo. Ravenna 6-14 marzo 1983 (1983) 237-243; altri esempi si posson trovare in Russo (nota 7) 132-240 passim; idem (nota 9) nn. 65. 67. 69. 172; C. Barsanti in: Guidobaldi - Barsanti - Guiglia Guidobaldi (nota 3) 138-150; E. Russo in: Pillinger (nota 11) 32-33; idem in: Romana pictura (nota 10) 74-75.

${ }^{86}$ Russo (nota 1) 14; Russo (nota 9) 19.

${ }^{87}$ Ed è rara pure al di fuori dei plutei: vd. C. Barsanti in: Guidobaldi - Barsanti - Guiglia Guidobaldi (nota 3) 155.

88 1) Salonicco, S. Giorgio (inv. BE 49), da S. Demetrio: medaglione in marmo bianco appartenente a un pluteo contenente un monogramma bordato, sormontato da una croce e posto entro un clipeo doppio, con una croce nel retro (diam. cm. 52; sp. cm.

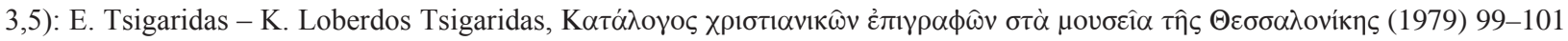
n. 71 figg. del recto e del verso a p. 100 (IV [?] sec.: ma è datazione sbagliata; con la bibl. precedente); D. Feissel, recensione all'opera precedente, Hellenika 32, 1980, 382-383 (dove si esclude la datazione al IV sec.); idem, Recueil des inscriptions chrétiennes de Macédoine du III ${ }^{\text {e }}$ au VI ${ }^{\text {e }}$ siècle (1983) 93 n. 94 tav. 21: Feissel scioglie il monogramma, sia pur in modo incerto,

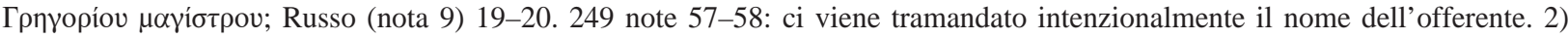
Salonicco, S. Demetrio: frammento di pluteo col medesimo monogramma, però non del tutto conservato: G. A. e M. G. Soteriou,

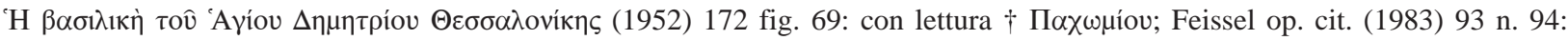
giustamente scarta la lettura di Soteriou; Russo (nota 9) 19-20. 249 note 57-58.

${ }^{89}$ F. Bulić, Scavi nelle basiliche urbane di Salona durante gli anni 1907, 1908, 1909, Bullettino di archeologia e storia dalmata 35, 1912, 20-21 tav. 2 (monogrammi di Onorio II); W. Gerber, Die Bauten im nordwestlichen Teile der Neustadt von Salona, Forschungen in Salona I (1917) 55 fig. 87; C. Barsanti in: Guidobaldi - Barsanti - Guiglia Guidobaldi (nota 3) 154-155 fig. 240 (oggi nel Museo archeologico di Spalato); C. Metzger - P. Chevallier in: Salona I. Catalogue de la sculture architecturale paléochrétienne de Salone (1994) 238-239 n. X.b.1 tavv. 77-78 (nel retro disco con croce; le autrici ritengono i pezzi importati, ignorando la problematica che ho sollevato nel 1984 e nel 1991).

90 C. Barsanti, Ancora sulla lastra con i monogrammi del monaco Giacinto, il presunto fondatore della Koimesis di Nicea, Università degli Studi di Roma La Sapienza. Annuario dell’Istituto di Storia dell’Arte n. s. 1, 1981/82, 8 fig. 5; eadem in: Guidobaldi - Barsanti - Guiglia Guidobaldi (nota 3) 154; Russo (nota 12) 3-4 fig. 14 (monogramma del probabile patrono, prova che l'opera è stata realizzata sul posto).
} 
d'edera (fig. 16), quest'ultima con paralleli soltanto in una lastra della chiesa di el-Felusyat (Ostracina) nel nord del Sinai $^{91}$ e in una della basilica E di Cnido ${ }^{92}$.

I plutei di S. Clemente son realizzati negli stessi anni del rifacimento giustinianeo di S. Sofia (532-537), e la maestranza è anche per la recinzione della chiesa romana di livello assai elevato. E non potrebb'esser altrimenti, trattandosi d'una commissione papale. Giunge ora a piena maturazione la sintassi compositiva, con un perfetto equilibrio dei motivi decorativi entro il campo riquadrato, saldamente da essi tenuto. Le caratteristiche formali son quelle proprie della migliore produzione greco-costantinopolitana coeva per i plutei ${ }^{93}$, e nella fattispecie la superficie di fondo non è affollata, ma è ampia e luminosa, in cui la luminosità è ottenuta mediante la levigatezza e la qualità del marmo, e in cui le parti a rilievo schiacciato e modulato emergon non per un gioco d'ombre, bensì come zone e risalti luminosi su fondi luminosi. È evidente che il materiale in marmo proconnesio dovette giunger a Roma tutt'al più sbozzato. Né vale invocar la presenza d'una lettera greca, del tutto isolata nel complesso di S. Clemente, un B su un frammento di architrave o di cornice $^{94}$, per affermare l'importazione dei pezzi già rifiniti da Costantinopoli ${ }^{95}$, in quanto il caso d'un capitello di Mut (Claudiopolis) in materiale locale ${ }^{96}$ - in cui pure compare la lettera $\mathrm{B}$, che ha ampia diffusione ${ }^{97}$ - ci indica in modo inequivocabile, nell'identica resa formale dei prodotti grecocostantinopolitani, soltanto la presenza in loco di maestranze greco-costantinopolitane, non d'un prodotto importato già rifinito. La medesima prudenza dobbiam applicare anche a complessi come quello di S. Clemente, pur se realizzati in marmo proconnesio, se di contro a tante sculture superstiti possiam leggere soltanto in un caso una lettera greca.

Ancora. A S. Clemente si trovano due plutei con schema ternario, costituito da due quadrati intrecciati affiancati da due rombi, dai vertici fioriti; entro i rombi è una croce latina a estremità patenti su un disco in funzione di globo; al centro in un caso un fiore quadripetalo, nell'altro - nella lastra che ho ricostituito con una parte oggi al Museo di Roma a palaz-

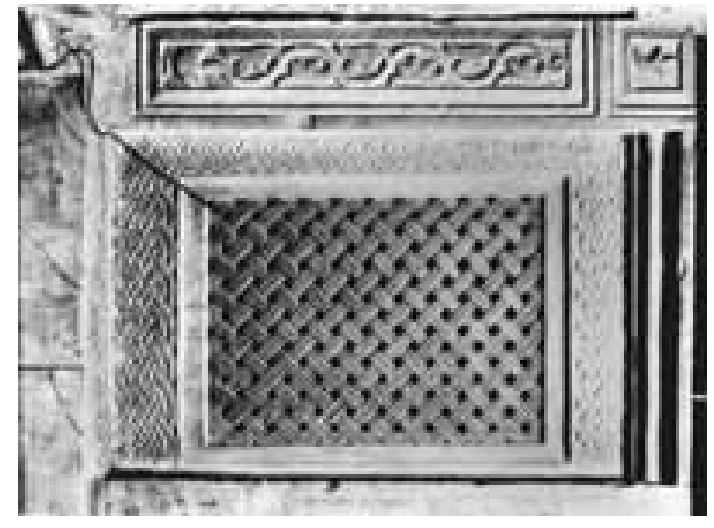

9 Roma, S. Clemente: transenna dell’altare del tempo di papa Ormisda

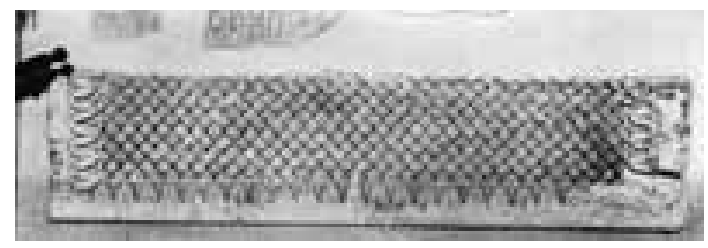

10 Roma, S. Agnese f. l. m.: frammento di pluteo col motivo a stuoia

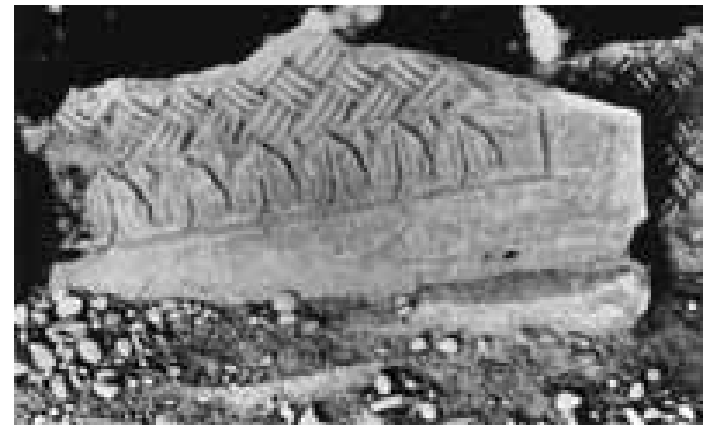

11 Roma, S. Agnese f. l. m.: frammento di pluteo col motivo a stuoia

\footnotetext{
${ }^{91}$ E. Oren, Un insediamento cristiano a Ostracina nel Sinai settentrionale [in ebraico], Qadmoniot 11 (1978) $84-85$ fig. a p. 85 a destra; Russo (nota 7) 235-237 fig. 84 (ritrovamento del 1976-77; la corona trova riscontro in un pluteo di S. Clemente; pezzo forse importato in Palestina già rifinito, forse realizzato almeno in parte in loco). Cf. C. Barsanti in: Guidobaldi - Barsanti - Guiglia Guidobaldi (nota 3) 149-150 fig. 233.

92 I. C. Love, Excavations at Knidos 1972, TürkAD 21, 2, 1974, 88 fig. 29; C. Barsanti in: Guidobaldi - Barsanti - Guiglia Guidobaldi (nota 3) 154 fig. 234.

${ }_{93}$ Per le caratteristiche dei plutei di S. Sofia rimando a Russo (nota 1) 16-19 figg. 5-6; idem (nota 6:1989) 608-610 fig. 4.

94 A. Guiglia Guidobaldi in: Guidobaldi - Barsanti - Guiglia Guidobaldi (nota 3) 233-238 figg. 369-370.

95 A. Guiglia Guidobaldi in: Guidobaldi - Barsanti - Guiglia Guidobaldi (nota 3) 239; C. Barsanti - A. Guiglia Guidobaldi in: Guidobaldi - Barsanti - Guiglia Guidobaldi (nota 3) 257-258; Sodini (nota 13) 434.

96 Sodini (nota 2:1979) 117 tav. 1 fig. 4.

${ }^{97}$ Deichmann (nota 2:1976) 221; J. P. Sodini, Marques de tâcherons inédites à Istanbul et en Grèce, in: Artistes, artisans et production artistique au moyen âge. Actes du Colloque international, Rennes, 1983 (1987) II 506-507; C. Barsanti in: Guidobaldi - Barsanti - Guiglia Guidobaldi (nota 3) 237 figg. 377-379.
} 


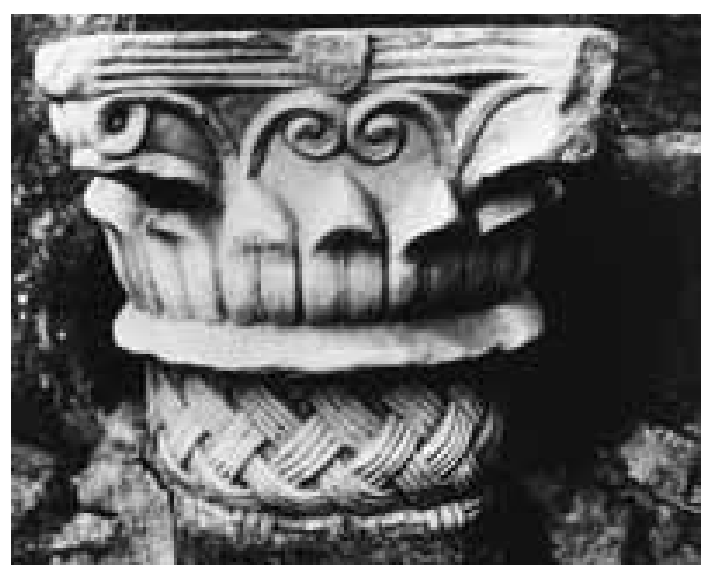

12 Roma, S. Agnese f. l. m.: capitello a paniere oggi nel Protettorato di S. Giuseppe

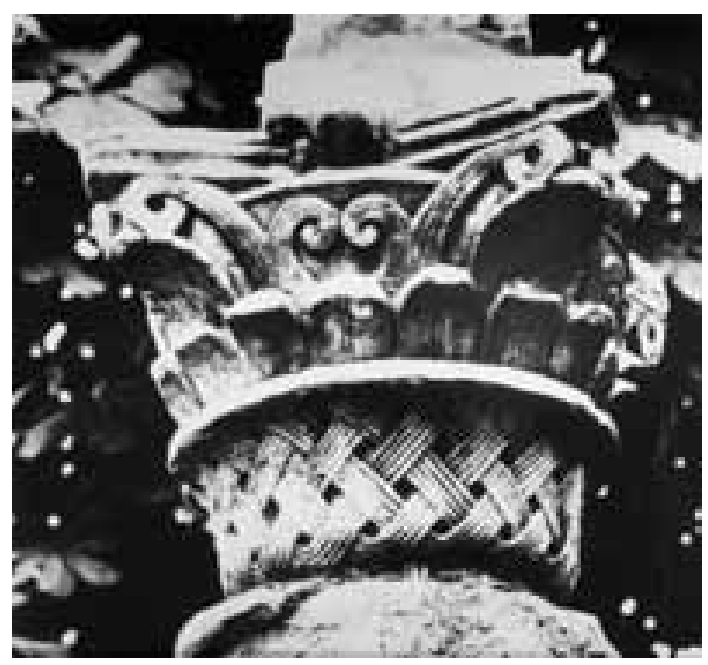

13 Roma, S. Agnese f. l. m.: capitello a paniere oggi nel Protettorato di S. Giuseppe zo Braschi (fig. 18) ${ }^{98}$ - un monogramma a 8 bracci. Ebbene, per quanto vicino a schemi presenti nella coeva S. Sofia, il nostro mostra la sua originalità, la sua unicità che non trova riscontri nella pur abbondantissima produzione della capitale e dell'ampio bacino dove tali prodotti son stati esportati99; e costituisce la manifestazione più originale (la più nuova $\mathrm{e}$ audace è data dall'inserimento del monogramma del committente nel gruppo che abbiam esaminato) tra le decorazioni realizzate dagli artefici costantinopolitani per Giovanni II a S. Clemente di Roma ${ }^{100}$.

La più originale per $\mathrm{S}$. Clemente, ma non in assoluto, perché la medesima maestranza ha eseguito per lo stesso papa la decorazione scultorea della chiesa dei Ss. Cosma e Damiano $^{101}$. Di tale decorazione ci colpiscono per la loro originalità assoluta non un frammento di pluteo con schema ternario come abbiam appena visto a S. Clemente (fig. 19) ${ }^{102}$, né un pilastrino della recinzione (fig. 20) ${ }^{103}$, pure analogo a quelli della medesima chiesa ${ }^{104}$, bensì i quattro capitellini del ciborio, oggi nel Tesoro della cattedrale di Lione - identificati nella loro provenienza da F. Guidobaldi -, a due zone come quelli del ciborio di Mercurio: nelle corone della zona superiore mostrano il monogramma a traforo del papa in origine due volte alternato a quello di un chrismon a 6 braccia per ciascun capitello (fig. 21), con uno straordinario festone dall'andamento sinuoso e continuo, il quale passa sotto le zampe delle aquile angolari dalle ali spiegate e nel suo incedere forma pure le corone che circondano i monogrammi. È ovvio che chi ha ritenuto importati già lavorati i marmi di S. Clemente ha espresso identica opinione per i pezzi dei Ss. Cosma e Damiano ${ }^{105}$. Ma i quattro capitellini presentano una serie di particolarità, che ne fanno un unicum, come evidenzia anche F. Guidobaldi, il quale pure è certo della loro esecuzione a Costantinopoli. Lo studioso ha dedicato

\footnotetext{
${ }_{98}^{98}$ Russo (nota 6:1989) 597-603 fig. 1; E. Russo, Frammento di pluteo in due parti, in: Aurea Roma (nota 6) 351 n. 352.

${ }^{99}$ Russo (nota 6:1989) 603-610. Non mi paiono cogenti le argomentazioni contrarie, favorevoli a vedere lo schema creato a Costantinopoli e non a Roma da parte di artefici costantinopolitani, addotte da C. Barsanti - A. Guiglia Guidobaldi in: Guidobaldi - Barsanti - Guiglia Guidobaldi (nota 3) 138. 257. Per altri esemplari dello schema a Roma vd. C. Barsanti in: Guidobaldi - Barsanti - Guiglia Guidobaldi (nota 3) 130-133 figg. 211-213.

${ }^{100}$ Desidero qui ricordare con gratitudine il dr. H. Sichtermann, direttore della Fototeca dell'Istituto Archeologico Germanico di Roma, che nel 1980 fece realizzare dietro mia richiesta una campagna fotografica delle sculture di S. Clemente: le fotografie dell'Istituto che presento sono frutto di quella campagna.

${ }^{101}$ C. Barsanti in: Guidobaldi - Barsanti - Guiglia Guidobaldi (nota 3) 132-133 fig. 212; A. Guiglia Guidobaldi in: Guidobaldi - Barsanti - Guiglia Guidobaldi (nota 3) 199 fig. 296; F. Guidobaldi, Origine costantinopolitana e provenienza romana di quattro capitelli del VI secolo oggi a Lione, MEFRA 101, 1989, 317-364 figg. 1-4.

102 C. Barsanti in: Guidobaldi - Barsanti - Guiglia Guidobaldi (nota 3) 132-133 fig. 212.

103 A. Guiglia Guidobaldi in: Guidobaldi - Barsanti - Guiglia Guidobaldi (nota 3) 199 fig. 296 (il pezzo era già noto al Mazzanti: 267 fig. 402 al centro).

104 Guidobaldi (nota 101) 317-364 figg. 1-4.

105 Guidobaldi (nota 101) 343-344; C. Barsanti - A. Guiglia Guidobaldi in: Guidobaldi - Barsanti - Guiglia Guidobaldi (nota 3) 256; J. P. Sodini in: Byzance. L'art byzantin dans les collections publiques françaises (Catalogue de l'exposition, Paris 1992) 40-41 n. 8; idem in: Sodini - Barsanti - Guiglia Guidobaldi (nota 2) 312; Melucco Vaccaro (nota 3:1999) 97 (»originali bizantini« [!]); Sodini (nota 13) 433; A. Guiglia Guidobaldi in: Christiana loca (nota 16) 268-269; C. Barsanti, Capitelli di manifattura costantinopolitana a Roma, in: Ecclesiae Urbis (nota 6) 1475-1477 fig. 20. Mi sono opposto a tale valutazione già in E. Russo in: Aurea Roma (nota 6) 196 fig. 5.
} 
ad essi un lungo studio, ma l'analisi del festone merita un approfondimento.

Io credo che debba escludersi dal confronto la ghirlanda che non sia frutto d'un festone continuo: per questo non posson esser presi in considerazione il frammentario capitello a due zone del Museo di Varna, che pure presenta le aquile angolari sopra la ghirlanda ${ }^{106}$; due capitelli compositi, non finiti, di Filippi ${ }^{107}$, con la ghirlanda pendente non sotto le volute angolari bensì nel kalathos tra le volute; cinque capitelli frammentarii della basilica della Campanopetra a Salamina di Cipro ${ }^{108}$, con il medesimo motivo. Di area greco-costantinopolitana con il festone continuo restano soltanto - non considerando un capitello nel lapidario del teatro di Perge ${ }^{109}$, perché di epoca romana - tre capitelli oggi nel Museo archeologico d'Istanbul ${ }^{110}$ e due da S. Marco di Venezia, l'uno nel chiostro di S. Apollonia ${ }^{111}$, l'altro nel Museo archeologico ${ }^{112}$ : i tre d'Istanbul hanno un festone per forma e andamento lontano dai capitellini oggi a Lione, i due di Venezia, corinzii, mostrano un festone più simile a quello dei pezzi oggi a Lione, passante in basso sotto le volute angolari e in alto dietro le aquile centrali a mo' di fiore d'abaco. Il festone continuo è invece più frequente in Siria ${ }^{113}$ : lo ritroviamo in un capitello della seconda metà del II - inizii III secolo, reimpiegato nella chiesa del V secolo di $\mathrm{Me}^{\prime} \mathrm{ez}^{114}$; in uno della chiesa, del 414 d. C., di Kseiğbe ${ }^{115}$; in uno della chiesa ovest, del 416 d. C., di Bāqirḥa ${ }^{116}$; in uno della chiesa di Qașr el Banāt, intorno al 430-440 ${ }^{117}$; in due della chiesa di Behyoo ${ }^{118}$, nella secon-

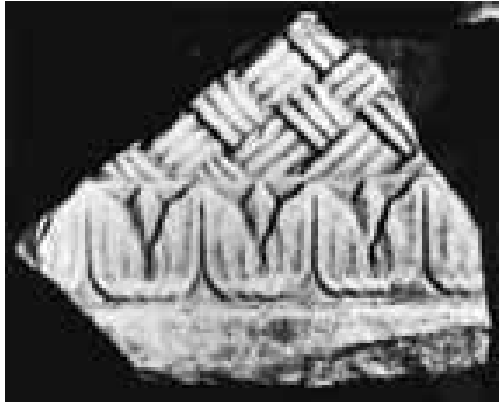

14 Roma, Museo Pio Cristiano Vaticano: frammento di pluteo col motivo a stuoia, da Porto

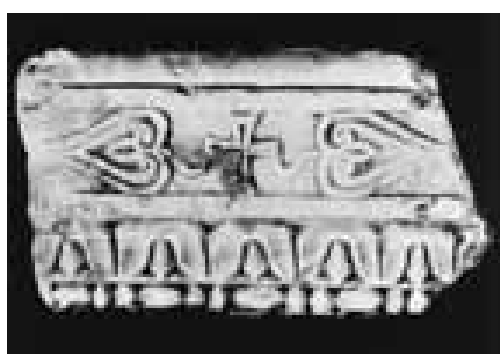

15 Roma, Museo Pio Cristiano Vaticano: frammento di pluteo

106 Guidobaldi (nota 101) 332-335 fig. 8; Barsanti (nota 19) 155-156 fig. 82.

107 M. Panayotidi, Quelques chapiteaux inachevés de Philippes, Byzantion 42, 1972, 424 fig. 3a e b; 424-425 fig. 4a. La Panayotidi non conosce altri esempi simili per il festone nei capitelli (428-430): i due di Filippi, in marmo locale, sono datati al VI sec. (430).

108 G. Roux, La basilique de la Campanopétra, Salamine de Cypre XV (1998) 111 figg. 145-149 (a p. 114 pensa ad artefici probabilmente venuti da Costantinopoli con il marmo; a p. 241 fig. 132, afferma che anche i pezzi in marmo del Proconneso eran importati allo stato di abbozzo [vi erano pure pezzi in calcare e in gesso]; a Cipro non c'era marmo, ch'era un prodotto di lusso); Sodini (nota 13) 431 fig. 13 (»Ces sculptures de la Campanopétra indiquent l’importation de marbre de Proconnèse et sans doute la venue de sculpteurs de la capitale.«); J. P. Sodini, La basilique de la Campanopétra en Cypre, JRA 13, 2000, 775-776 fig. 7 (ora Sodini, 776. 778, parla di ateliers regionali, forse della Cilicia, che ben conoscono il repertorio di Costantinopoli).

${ }^{109}$ U. Peschlow, Tradition und Innovation: Kapitellskulptur in Lykien, in: Spätantike und byzantinische Bauskulptur (nota 47$) 71$ tav. 21, 19. Festone continuo, che passa sotto le volute angolari ed è retto in alto al centro dei lati da un putto.

${ }^{110}$ Firatl1 (nota 51) nn. 227-229.

${ }^{111}$ F. W. Deichmann, Corpus der Kapitelle der Kirche von San Marco zu Venedig (1981) 145, A 12 tav. 48.

112 Ibidem 145-146, M 1 tav. 49.

${ }^{113}$ In Siria è naturalmente presente anche il festone a ghirlanda non continuo: vd. ad es. capitelli di Gerasa, Museo (Kautzsch [nota 48] 140-141 n. 456 tav. 28: III sec.); di Bābisqā, chiesa est (G. Tchalenko, Églises syriennes à bêma [1990] 115; 1979 [Planches], tav. 298 in alto; 1980 [Album], fig. 168 in alto a destra [116-117: fine IV - inizi V sec.]; Ch. Strube, Baudekoration im nordsyrischen Kalksteinmassiv I (1993) 50 tav. 18f [49: 390-404 d. C.]); di Kseiğbe, chiesa (ibidem 58-59 tav. 21a [57: 414 d. C.]); di El Bara, chiesa principale (ibidem 175 tavv. 70a. 71c [178-180: seconda metà V sec.]); di Resafa, edificio a pianta centrale (G. Brands, Die Entstehung einer Stadt. Beobachtungen zur Bauornamentik von Resafa, in: Spätantike und byzantinische Bauskulptur [nota 47] 83-85 tav. 25, 7; idem, Die Bauornamentik von Resala-Sergiupolis, Resafa VI [2002] 139-140. 171. 178-179 Beil. $25 \mathrm{a}$ e b tav. 51e ed f [primo quarto del VI sec., prima del 520, poco dopo il 510]; Ch. Strube, Baudekoration im nordsyrischen Kalksteinmassiv II [2002] 38. 41. 108-109 tav. 28c); di Mayâfârqîn, basilica (G. Bell in: M. Mundell Mango [ed.], The Churches and Monasteries of the Țur 'Abdin [1982] 58-60 tav. 42); di Nișîbîn, Mâr Ya'qûb (ibidem 70-74 tav. 80); di Arnas, Mâr Kyriakos (ibidem 15-17 tav. 103); di Ḥâkh, el 'Adhra (ibidem 54-58 tavv. 139. 141. 143. 145); di Kefr Zeh, Mâr Azîzael (ibidem 44-47 tavv. 159. 161; la chiesa però è tarda: cf. M. Mundell Mango in: ibidem 120-121).

114 Strube (nota 113:2002) 10-11 tav. 2b.

115 Strube (nota 113:1993) 58-59 tav. 21b (57: 414 d. C.).

116 Tchalenko (nota 113) 132-133 fig. 197.

117 Strube (nota 113:1993) 64 tav. 25c e d (per la datazione, p. 66).

118 Tchalenko (nota 113) 158 fig. 263 al centro (159: metà del V sec.); Strube (nota 113:1993) 88 tav. 37a (137: tra il 470 e il 480). 


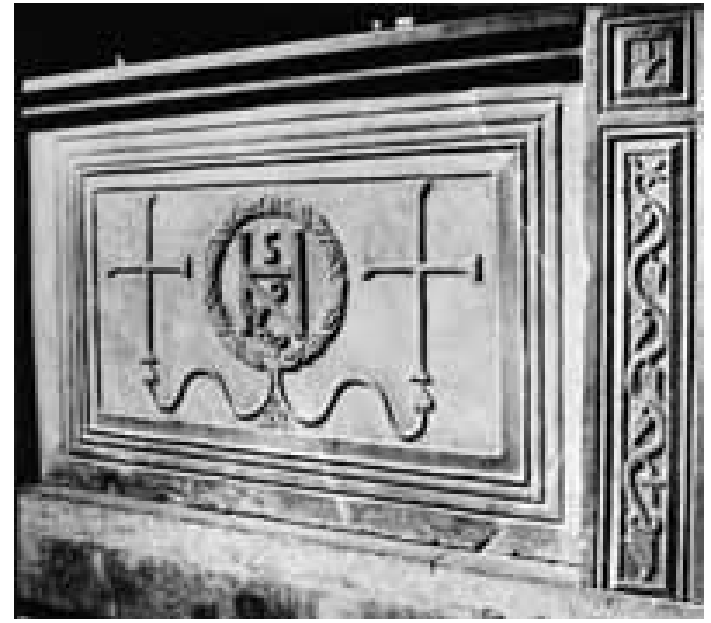

16 Roma, S. Clemente: pluteo con il monogramma di papa Giovanni II (533-535)

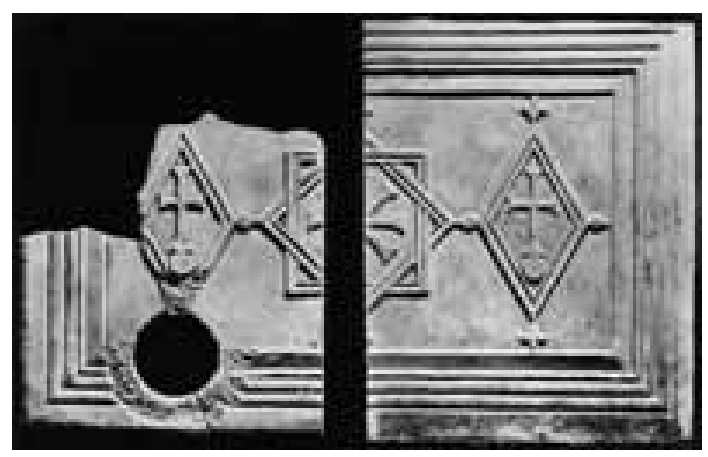

18 Roma, S. Clemente e Museo di Roma: pluteo frammentario, ricomposto mediante fotomontaggio

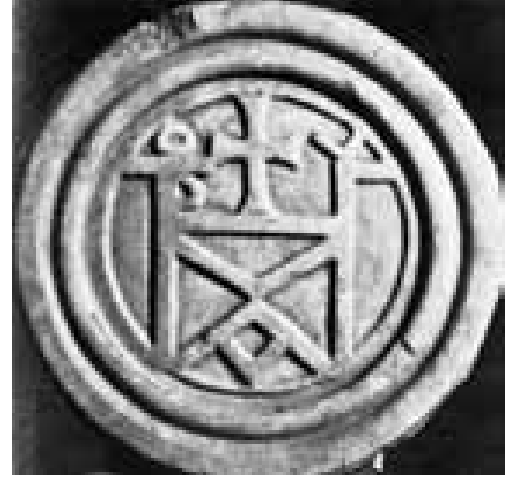

17 Salonicco, S. Giorgio: medaglione di pluteo, proveniente da S. Demetrio

da metà del V secolo; in uno della chiesa di Dēhes, nella seconda metà del $\mathrm{V}$ secolo ${ }^{119}$; nei capitellini dell'iconostasi di Mâr Azîzael a Kefr Zeh, che però è chiesa $\operatorname{tarda}^{120}$. Ma la particolarità dei capitelli di Lione consiste nel fatto che il festone disegna nel suo percorso continuo pure le corone dei monogrammi. Ebbene, ancor una volta la Siria ci viene in aiuto: infatti in un capitello di colonna della chiesa est di Kaukanāyā, del VI secolo (fig. 22) ${ }^{121}$, non soltanto il festone è continuo, ma in corrispondenza del centro dei quattro lati in alto forma con il nastro un occhiello. È l'esempio più vicino ai capitelli del ciborio di Giovanni II che io conosca, cui può aggiungersi il capitello di colonna reimpiegato nell'arcata nord dell'Ulu Camii a Diyarbakır (fig. 23) ${ }^{122}$, dove il festone al centro in alto forma un piccolo occhiello; e pure un capitello frammentario di colonna del Museo di Urfa ${ }^{123}$, in cui il festone pare disegnar una corona in alto al centro (sembra intorno al fiore d'abaco). In Siria vi sono pure delle varianti del motivo: un capitello di colonna, reimpiegato nella facciata ovest dell'Ulu Camii a Diyarbakir ${ }^{124}$, e uno reimpiegato nell'arcata nord ${ }^{125}$, hanno il festone con un doppio nodo al centro in alto, così come un capitello di pilastro nel Museo di Urfa ${ }^{126}$; oppure la presenza del doppio nodo si accompagna a una sorta di clipeo soprastante formato dal nastro del festone e contenente un fiore a 6 petali, come nei due capitelli absidali di pilastro, di nord ${ }^{127}$ e di sud (fig. 24) ${ }^{128}$, nella chiesa di S. Maria a Diyarbakır; oppure il capitello di pilastro reimpiegato nella grande moschea di Ḥarrān ${ }^{129}$, dove vediamo una sorta di doppio nodo (non preciso), da cui si diparte un nastro che isolato costituisce un clipeo intorno al fiore d'abaco sopra il nodo ${ }^{130}$.

119 Tchalenko (nota 113) 136 tav. 347 in basso, fig. 215 in alto a sinistra (137: verso la metà del V sec.); Strube (nota 113:1993) 123 tav. 49b (datazione 134-138).

120 G. Bell in: Mundell Mango (nota 113) 44-47 tavv. 162-163 (e per la datazione cf. M. Mundell Mango in: ibidem 120-121).

${ }^{121}$ Strube (nota 113:2002) 132 tav. 96a e b (131: la chiesa è del VI sec.).

122 Brands (nota 113:2002) 171. 248. 255. 256. 266 tav. 91b (247: intorno al 500 per B.).

123 Brands (nota 113:2002) 240-241 tav. 82b.

124 Brands (nota 113:2002) 171. 248. 255. 256. 266 tav. 87c (247: intorno al 500 per B.).

125 Brands (nota 113:2002) 171. 248. 255. 256. 266 tav. 91a (247: intorno al 500 per B.).

126 Brands (nota 113:2002) 240-241 tav. 82d (240: intorno al 520 per B.).

127 Brands (nota 113:2002) 171. 191. 247-249. 258. 267 tavv. 92d ed e; 93a (248: primo terzo del VI sec. per B.).

128 Brands (nota 113:2002) 171. 191. 247-249. 258. 267 tav. 93b (248: primo terzo del VI sec. per B.).

129 Brands (nota 113:2002) 241-242 tav. 83b (242: inizi VI sec. per B.).

${ }_{130}$ D'altra parte una corona sorge da e sopra un nastro a doppio nodo nel portale centrale della facciata ovest della chiesa sud di Ruweiḥa (A. Naccache, Le décor des églises de villages d'Antiochène du IV au VII siècle I [1992] 252-253 figg. 178. 284; II 
I quattro capitelli oggi a Lione mostrano comunque caratteristiche tali da esser posti nell'insieme con una connotazione di singolarità nel quadro greco-costantinopolitano successivo al S. Polieucto e contemporaneo ai Ss. Sergio e Bacco e alla S. Sofia. Io credo che tale singolarità sia frutto anche d'un'osservazione e d'una rivisitazione autonoma e del tutto originale di quanto era davanti agli occhi nei monumenti classici di Roma (altari, sarcofagi, etc.) da parte di artefici che avevan presenti sia il repertorio costantinopolitano sia i modelli siriaci e che hanno elaborato appositamente per Giovanni II la decorazione, con le corone centrali in alto a contenere i monogrammi. È impensabile una lavorazione dei pezzi - che hanno nei monogrammi il fulcro della composizione, su loro incentrata - a Costantinopoli e non a Roma.

È evidente che mi concentrerò ora non sui prodotti importati già rifiniti, peraltro indicati già nel saggio pubblicato nel $1984^{131}$, bensì sulle opere eseguite a Roma da artefici greco-costantinopolitani.

Un pulvino conservato nella catacomba dei Ss. Marcellino e Pietro (fig. 25) ${ }^{132}$ si presenta come un pezzo importato cui manca soltanto l'ultima rifinitura. Però non è così: si tratta d'una scultura realizzata da maestranze greco-costantinopolitane ${ }^{133}$, com'è dimostrato dalle sue caratteristiche non soltanto tipologiche ma anche formali (come l'abbassa-

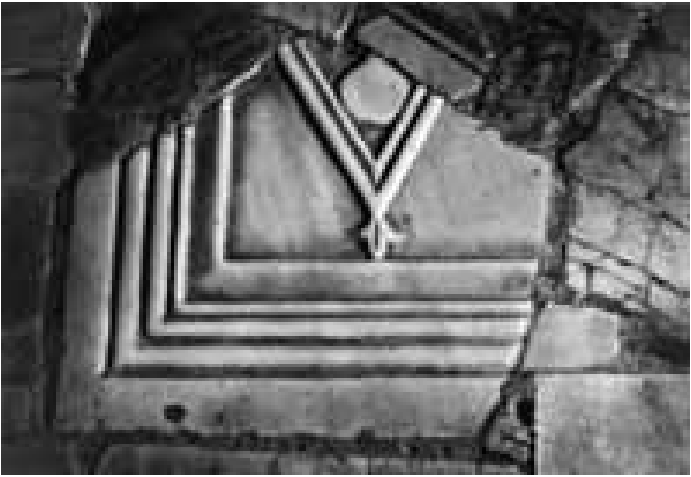

19 Roma, Ss. Cosma e Damiano: frammento di pluteo del tempo di Giovanni II

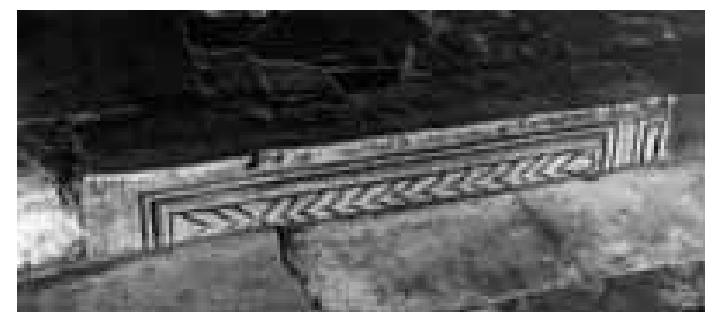

20 Roma, Ss. Cosma e Damiano: pilastrino frammentario di recinzione del tempo di Giovanni II mento del fondo lungo i bordi della decorazione), e a Roma, dato che il pulvino è stato ricavato da un blocco di reimpiego d'un'iscrizione monumentale con tracce di lettera ${ }^{134}$. È istruttivo confrontarlo con l'attuale fianco sinistro del sarcofago dell'arcivescovo Grazioso († 788) in S. Apollinare in Classe (fig. 26), decorato con l'attuale retro alla metà circa del VI secolo a opera di maestranze greco-costantinopolitane, nella prima rilavorazione cristiana della cassa d'un sarcofago pagano ${ }^{135}$. La

tav. 24) e nel portale sud-ovest della basilica di Qalblōze (Tchalenko [nota 113] 165 figg. 280-281: súbito dopo la metà del V sec.; Naccache op. cit. 252-253 fig. 229; Strube [nota 113:1993] 103 tav. 45d [105-115: 459-470]).

${ }^{131}$ Russo (nota 1) rispettivamente 19. 23-24 per le opere in cui lasciavo aperta la possibilità d'una lavorazione a Roma o d'un'importazione diretta di prodotti già rifiniti (»presenza di maestranze e/o prodotti greco-cost/i«), e 19-22 per le sculture che già allora mi apparivano prodotti importati già rifiniti (»prodotti standard«).

132 O. Marucchi, La cripta storica dei Ss. Pietro e Marcellino recentemente scoperta sulla via Labicana, Nuovo Bullettino di archeologia cristiana 4, 1898, 158-159 fig. 3 (VI sec., nel restauro della catacomba da M. attribuito al papa Vigilio); J. Guyon, Le cimitière 'Aux deux lauriers'. Recherches sur les catacombes romaines (1987) 450-452 figg. 255 a destra e 256 (Guyon pensa, a proposito della basilica ad corpus, in cui il pulvino sarebbe stato messo in opera, a un lavoro di papa Onorio, 625-638: 452-455); A. Augenti, Ipsi lapides ululant nobiscum. Il suburbio orientale di Roma tra la tarda antichità e l'alto medioevo, Melanges de l'École française de Rome. Moyen âge - temps modernes 103, 1, 1991, 62-64 figg. 10-11 (VI sec., epoca di papa Vigilio): estemporanea è l’osservazione di A. su »la laconicità dell'ornato« (62).

133 È in errore Augenti (nota 132) 63-64, a proporre un confronto con i plinti di S. Lorenzo f. l. m., essendo questi opere romane, e a ritenere il pulvino produzione romana, ed è del pari sbagliato affermare che i motivi decorativi »sembrano derivati da opere in metallo; è una caratteristica tipica della produzione scultorea romana di VI secolo« (64).

134 Guyon (nota 132) 450 e nota 127; Augenti (nota 132) 62 nota 63.

135 È questa la conclusione cui son giunto in E. Russo, Studi sulla scultura paleocristiana e altomedievale, pubblicati dapprima in edizione separata (1974) e súbito dopo comparsi in Studi medievali terza serie 15, 1, 1974, rispettivamente 1-6. 114-115 e 25-30. 138-139 tav. 2a (intorno alla metà del VI sec.); conclusione non conosciuta da H. Herdejürgen in: J. Kollwitz - H. Herdejürgen, Die ravennatischen Sarkophage (1979) 16. 51 tav. 88, 1, che continua a datare alla morte di Grazioso, 788, tutta l'attuale decorazione del sarcofago; conclusione invece accolta, in modo un po' contorto, da Deichmann (nota 2:1989) 346. 
parentela tecnica e formale è talmente stretta, che pure per il pulvino nella catacomba si può proporre una datazione intorno alla metà del VI secolo ${ }^{136}$.

Nelle porte delle mura urbiche ritroviamo pure, per le chiavi di vôlta degli archi, una serie di croci o monogrammi, incisi o scolpiti: ciò avviene per le porte Pinciana (fig. 27) ${ }^{137}$, Latina (fig. 28) ${ }^{138}$, Appia (fig. 29) $)^{139}$ e Ostiense (fig. 30) $)^{140}$. Se la croce latina sull'arco interno della porta Pinciana mostra estremità patenti (fig. 27), e per tal guisa potrebbe pur appartenere all'epoca di Onorio, cui generalmente si attribuiscono i lavori alle mura e le decorazioni ora esaminate ${ }^{141}$, sull'arco esterno la croce greca entro un clipeo (fig. 27), ottenuti entrambi a incisione, ci rimanda invece a un'epoca successiva e c'introduce in un mondo
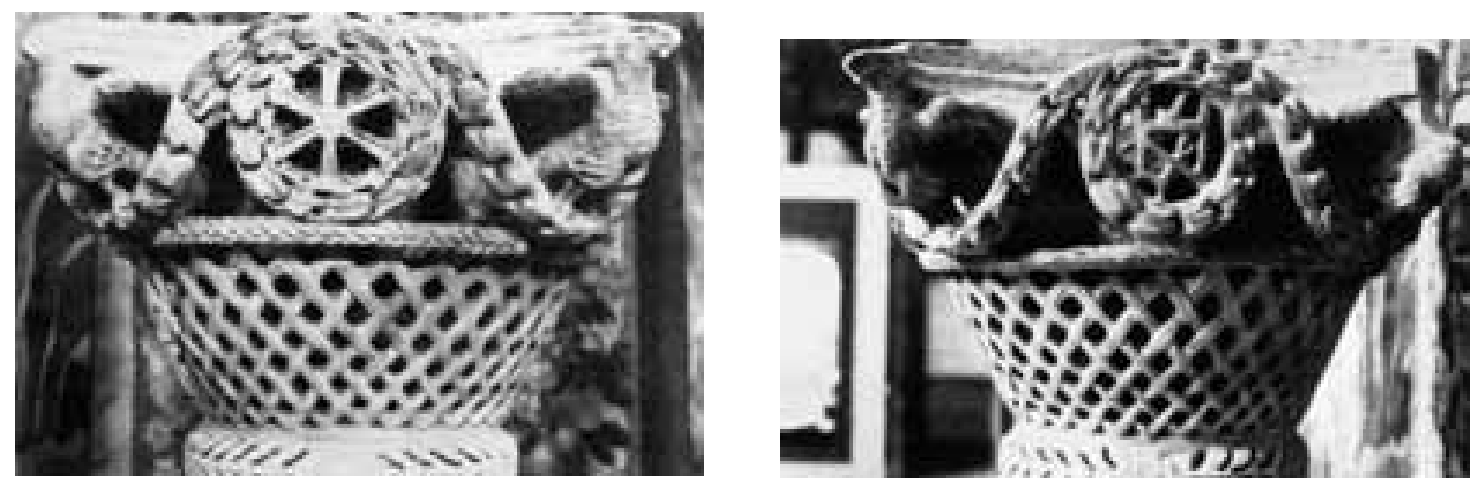

21 Lione, Tesoro della cattedrale: uno dei quattro capitellini del ciborio di Giovanni II, provenienti dalla chiesa dei Ss. Cosma e Damiano di Roma
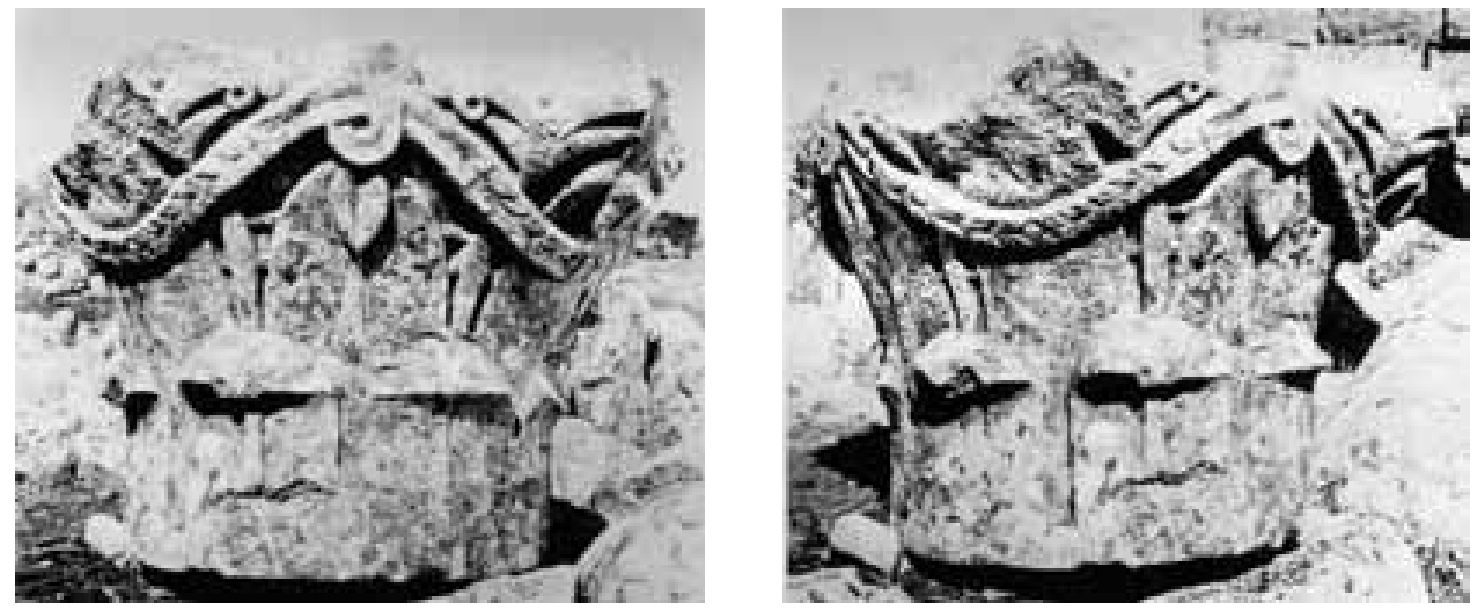

22 Kaukanāyā, chiesa est: capitello di colonna

\footnotetext{
136 Tale datazione si basa sull'esame del pezzo, e prescinde dalla presunta opera del papa Vigilio, che A. Ferrua, I lavori del papa Vigilio nelle catacombe, Civiltà Cattolica 118, 2, 1967, 142-148 (= Scritti vari di epigrafia e antichità cristiane [1991] 332-338), ha dimostrato non veritiera per la catacomba dei Ss. Marcellino e Pietro.

${ }^{137}$ G. B. Giovenale, Simboli tutelari su porte del recinto urbano ed altri monumenti dell'antichità, BCom 57, 1929 (1930) 257-258 fig. 48 in basso; I. A. Richmond, The City Wall of Imperial Rome (1930) 107 fig. 18C e D; Broccoli (nota 69) 83-84 n. 10 tav. 4, 1 e 2; L. Cozza, Osservazioni sulle mura aureliane a Roma, AnalRom 16, 1987, 26-29 fig. 12a e b.

138 Giovenale (nota 137) 257-258 fig. 48 in alto a destra; Richmond (nota 137) 107 fig. 18B e G; Cozza (nota 137) 26-29 fig. 13a e b.

139 Giovenale (nota 137) 257-258 fig. 48 in alto a sinistra (legge correttamente l'epigrafe); Richmond (nota 137) 107-108 fig. 18A (legge erroneamente $\Theta \mathrm{E} \omega$ in luogo di $\Theta \mathrm{EOY}$ ); Cozza (nota 137) 26-29 fig. 14a (Cozza riproduce pure alcune croci a rilievo sulla cortina marmorea esterna, alla destra dell'arco, fig. 14b, e una croce incisa alla base della torre orientale).

140 Richmond (nota 137) 107 fig. 18E ed F; Cozza (nota 137) 26-29 fig. 15a e b.

${ }^{141}$ Richmond (nota 137) 107-108; Cozza (nota 137) 26-49; R. Mancini, Le mura aureliane di Roma. Atlante di un palinsesto murario (2001) 26-35; A. Guiglia Guidobaldi in: Ecclesiae Urbis (nota 6) 1502 (»pertinenti probabilmente già al restauro di Onorio del V secolo«).
} 
sicuramente non occidentale, ma nemmeno proprio di Costantinopoli, giacché le braccia progressivamente patenti e svasate si ritrovan nel mondo della provincia microasiatica, dell'Egitto copto e della Siria, in »quella koเvì artistica situata nelle regioni orientali del Mediterraneo « ${ }^{142}$, cui sono da riferire pure le croci a braccia fortemente patenti. La tecnica a incisione ci rimanda all'attuale tergo del già ricordato sarcofago dell'arcivescovo Grazioso (fig. 31) ${ }^{143}$ con medesime maestranze e datazione dell'attuale fianco sinistro e ad opere all'incirca coeve quali una lastra del Museo di Mileto (fig. 32) ${ }^{144}$, le lastre d'una fontana in una delle botteghe di Sardi ${ }^{145}$, i parapetti dell'ambone della basilica 'delle tre chiese’ a Paro $^{146}$, i parapetti della fontana sulla via dei Cureti a Efeso $^{147}$. Veramente calzante per forma della croce nel clipeo e per tecnica d'esecuzione è il confronto con la lastra di Mileto (fig. 32), e per la forma della croce nel clipeo con uno dei parapetti della fontana di Efeso ${ }^{148}$, così che ne resta assicurata la data intorno alla metà del VI secolo e la mano d'una maestranza molto probabilmente microasiatica. In tal modo

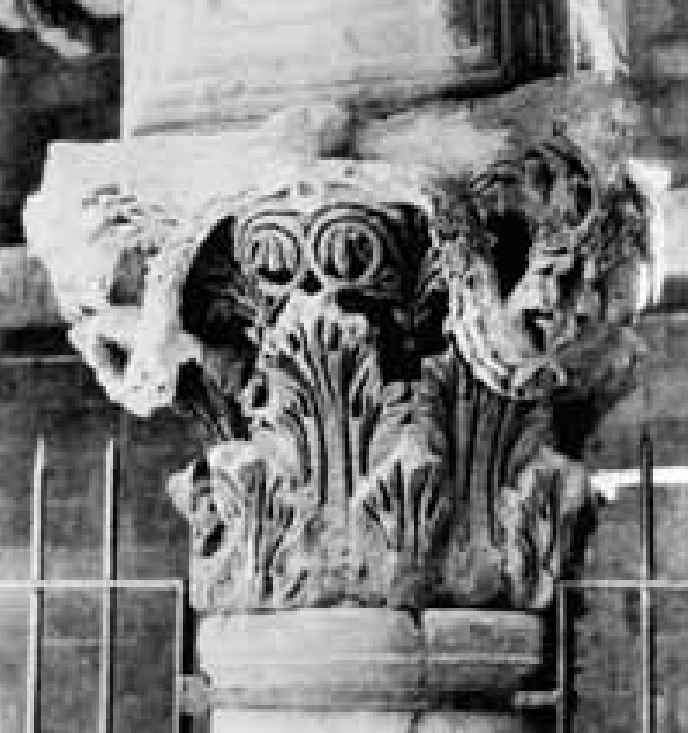

23 Diyarbakır, Ulu Camii: capitello di colonna reimpiegato nell'arcata nord si evidenzia che le decorazioni son dovute a Narsete, alla fine delle guerre gotiche. Una convinzione che si rafforza nella considerazione della croce nella chiave di vôlta interna dell'arco, non del monogramma cristologico con X e P lettere apocalittiche nella chiave di vôlta esterna della porta Latina (fig. 28); e che diventa certezza guardando la chiave di vôlta interna dell'arco di porta Appia (fig. 29), dove la croce greca incisa in un cerchio inciso, dalle braccia fortemente e progressivamente patenti e leggermente svasate, è accompagnata dalla scritta in greco

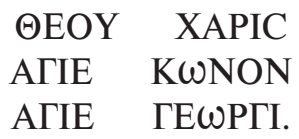

Una scritta in greco, e con invocazione a due santi sconosciuti o quasi prima della conquista bizantina della città, è veramente assurda per l'epoca di Onorio ${ }^{149}$. La porta Appia ci mostra pure una croce incisa alla base della torre orientale (fig. 29) e alcune croci a rilievo sulla cortina marmorea esterna, alla destra dell'arco. Menziono infine una croce greca incisa in un cerchio nella chiave di vôlta esterna dell'arco di porta Ostiense (fig. 30), quasi identica a quella di porta Appia, e nella chiave interna un'incisione imprecisa, con un cerchio approssimativo contenente un motivo a mezza strada tra una croce e un monogramma (fig. 30). Son tutti lavori da ricollegare a restauri o comunque a interventi del tempo di Narsete.

D’altra parte nella chiesa dei Ss. Apostoli, sorta al tempo di Pelagio I (556-561) ${ }^{150}$ e portata a termine sotto Giovanni III (561-574) ${ }^{151}$, troviamo importanti conferme della presenza a Roma di maestranze greco-

\footnotetext{
142 Russo (nota 135) rispettivamente 46 e 70 (per tali tipi di croci vd. 29. 45-49=53. 69-73 tav. 9).

${ }_{143}$ Russo (nota 135) tav. 3 (e per il fenomeno dell'incisione, 49-118 = 73-142 tavv. 13-40).

144 E. Russo in: Pillinger (nota 11) 35 fig. 20.

145 E. Russo in: Pillinger (nota 11) 35 (con la bibl. precedente a nota 73).

${ }^{146}$ E. Russo in: Pillinger (nota 11) 35 (con la bibl. precedente a nota 74).

147 E. Russo in: Pillinger (nota 11) 34-35 figg. 12-19.

148 E. Russo in: Pillinger (nota 11) fig. 13.

149 Prive di valore le argomentazioni di Richmond (nota 137) 108, a favore dell'epoca di Onorio.

${ }^{150}$ L. Duchesne (ed.), Liber Pontificalis I (Paris 1886) 303.

${ }^{151}$ Ibidem 305.
} 


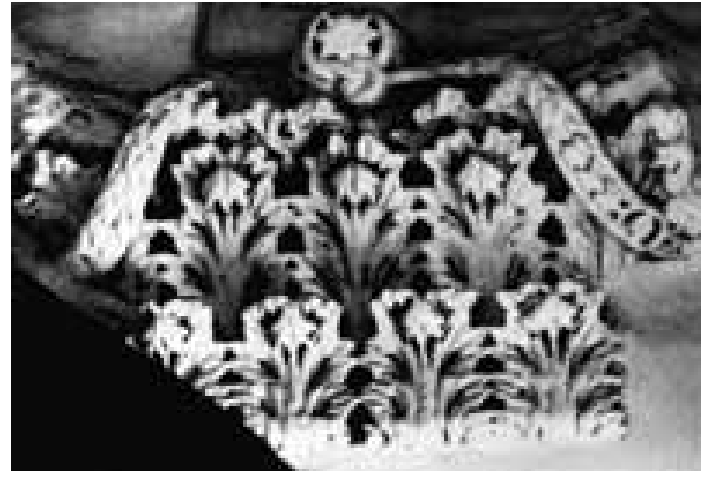

24 Diyarbakır, S. Maria: capitello absidale del pilastro di sud
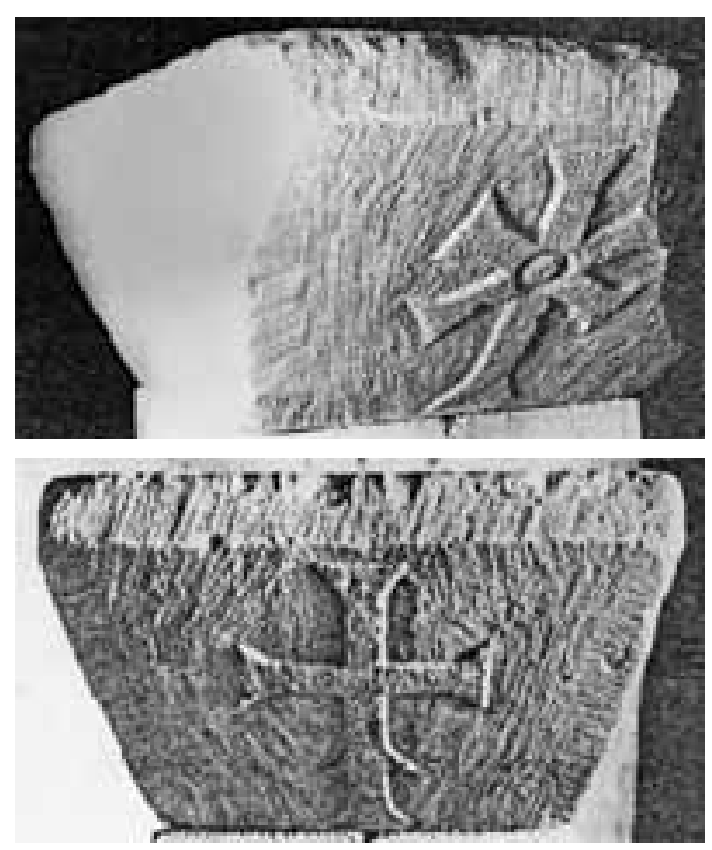

25 Roma, catacomba dei Ss. Marcellino e Pietro: pulvino costantinopolitane di alto livello anche ben dopo la fine delle guerre gotiche, come l'altare a cassa con i quattro pilastrini angolari e la mensa di pavonazzetto (fig. 33), costituita da due pezzi distinti di ricupero (le venature dei due pezzi non corrispondono) $^{152}$, rilavorati per l'occasione da maestranze greco-costantinopolitane al tempo di Giovanni III con una sottile croce dalle lunghe braccia rese con un raffinato incavo interno a modanature, e in modo da campire l'intero spazio interno entro l'incorniciatura sagomata e modanata (fig. 34); e un pilastrino superstite della recinzione, oggi murato nel portico (fig. 35), già noto a F. Mazzanti ${ }^{153}$. Ora il pilastrino, le cui caratteristiche formali rimandano indubitabilmente all'area greco-costantinopolitana, è stato scolpito certissimamente a Roma, dato che è in marmo lunense, di Carrara. Ciò ha come conseguenza che l'intera recinzione dei Ss. Apostoli, oltre all'altare, è stata eseguita a Roma. Si verifica dunque un fenomeno ben diverso da quello ipotizzato dalla critica ${ }^{154}$, vale a dire la semplice importazione di pezzi di proconnesio già rifiniti, dato che le opere che ho presentato indicano anche l'attività a Roma di maestranze greco-costantinopolitane, le quali in assenza del marmo proconnesio rilavorano il marmo trovato sul posto.

È un quadro assai più ricco e articolato, che vede come tappa importante i parapetti e i pilastrini del ponte Salario sull'Aniene, rifatto da Narsete e precisamente datato da un'epigrafe al $565^{155}$. V. Galliazzo lo ha ricostruito graficamente con 24 pilastrini e 11 lastre (di cui la centrale contenente un'epigrafe) per lato ${ }^{156}$. Sono note le vicende del ponte, che ha subíto danni e distruzioni documentate dal 1798 al 1867, e oggi del tutto privo della decorazione del VI secolo. Fortunatamente se ne posseggono varii disegni e incisioni, che ho cercato di raccogliere e vagliare e che ci danno una visione sia pur parziale delle decorazioni. I principali ai nostri fini posson esser indicati in un'incisione di Duflos

152 I. Mazzucco, Filippo e Giacomo apostoli nel loro santuario romano. Il sepolcro, le reliquie, il culto (1982) 20-30 figg. 4-11; E. Russo in: Aurea Roma (nota 6) 196; G. Bartolozzi Casti, Il sarcofago dei Maccabei e gli altari-sarcofago dei Ss. Apostoli e dei Ss. Cosma e Damiano, RendPontAc 72, 1999/2000 (2001) 195-196 fig. 5; F. Guidobaldi in: Materali e tecniche (nota 17) 179 fig. 9.

153 Vd. A. Guiglia Guidobaldi in: Guidobaldi - Barsanti - Guiglia Guidobaldi (nota 3) 268. Il pilastrino è stato pubblicato dalla Guiglia, ibidem 200 fig. 300, con la preziosa indicazione della qualità del marmo, il lunense. Ma non riesco a condivider la sua valutazione, rispetto a S. Clemente, "per la forma più rigonfia delle foglie e per l'aspetto più naturalistico del tralcio stesso che rinviano, forse ancor più esplicitamente, a modelli di tradizione classica« (213-214).

154 Non potendo negare l'evidenza per il pilastrino dei Ss. Apostoli in marmo lunense, ma evidentemente attaccate alla loro idea che i pezzi lavorati dai greco-costantinopolitani fossero giunti a Roma già rifiniti, C. Barsanti e A. Guiglia Guidobaldi scrivono: "Resta invece opinabile l'identità del suo artefice: si trattava di uno o più scultori provenienti da Costantinopoli, oppure di maestranze romane come potrebbe eventualmente segnalare l'allure classicheggiante del tralcio d'edera?« (Guidobaldi - Barsanti Guiglia Guidobaldi [nota 3] 263). È un dilemma che non ha motivo d'esistere e che appare formulato per sostenere una ricostruzione dei fatti inadeguata alla verità, ma che viene riproposto in A. Guiglia Guidobaldi in: Christiana loca (nota 16) 269, e in eadem in: Ecclesiae Urbis (nota 6) 1491 (e nelle incertezze sulla presenza »di maestranze provenienti dalla capitale«, 1482-1483, si conclude che »Ciò sembrerebbe rafforzare l'ipotesi secondo la quale l'arrivo a Roma di plutei e pilastrini di marmo proconnesio in numero davvero rilevante costituisca un fenomeno cronologicamente piuttosto ristretto e con tutta probabilità precedente alle guerre gotiche«, 1483).

155 CIL VI 1199; cf. E. Diehl, Inscriptiones latinae christianae veteres I (1925) 23 n. 77. Per la bibl. sul ponte fino al 1979 vd. Russo (nota 1) 22-23; dopo il 1979, A. Guiglia Guidobaldi in: Christiana loca (nota 16) 1491-1502 figg. 4-7.

156 V. Galliazzo, I ponti romani II (1994) 45-48 n. 32 figg. a 46 e 47. 
pubblicata da Ridolfino Venuti nel $1744^{157}$, in una di Anonimo uscita nel $1748^{158}$, nella celeberrima del Piranesi del 1754 (fig. 36) ${ }^{159}$, in una di Anonimo pubblicata nel $1796^{160}$, nei disegni preparatorii (fig. 37) e nelle incisioni pubblicate dal Séroux d'Agincourt (fig. 38) ma frutto di lavori anteriori al $1798^{161}$, in un'incisione pubblicata da A. Uggeri nel $1810^{162}$, in una pubblicata da G. B. Cipriani nel $1817^{163}$, in un acquarello del 1820 di M. Dubourg ${ }^{164}$, in un'incisione di L. Rossini del $1822^{165}$, in un disegno a matita di Francesco Caracciolo del 1826 (fig. 39) ${ }^{166}$, in un disegno acquarellato di Anonimo, realizzato dopo la distruzione della torre nel $1829^{167}$, eseguito poco dopo il 1843 (fig. 40) ${ }^{168}$, che ci mostra il lato est, faccia verso est, senza la torre e alcuni dettagli dei parapetti e dei pilastrini del lato est, faccia verso ovest, fino al 1829 coperti dalla torre.

E dall'esame di questa documentazione ${ }^{169}$ emerge un quadro assai differente da quel ch'è stato ipotizzato, secondo cui »ben diversa è la direzione che prese la produzione romana nella seconda metà del VI secolo « ${ }^{170}$ rispetto alla produzione greco-costantinopolitana. Infatti in 5 dei parapetti noi vediamo uno schema decorativo tripartito che sicuramente non è di origine romana, ma non trova riscontro nemmeno nella

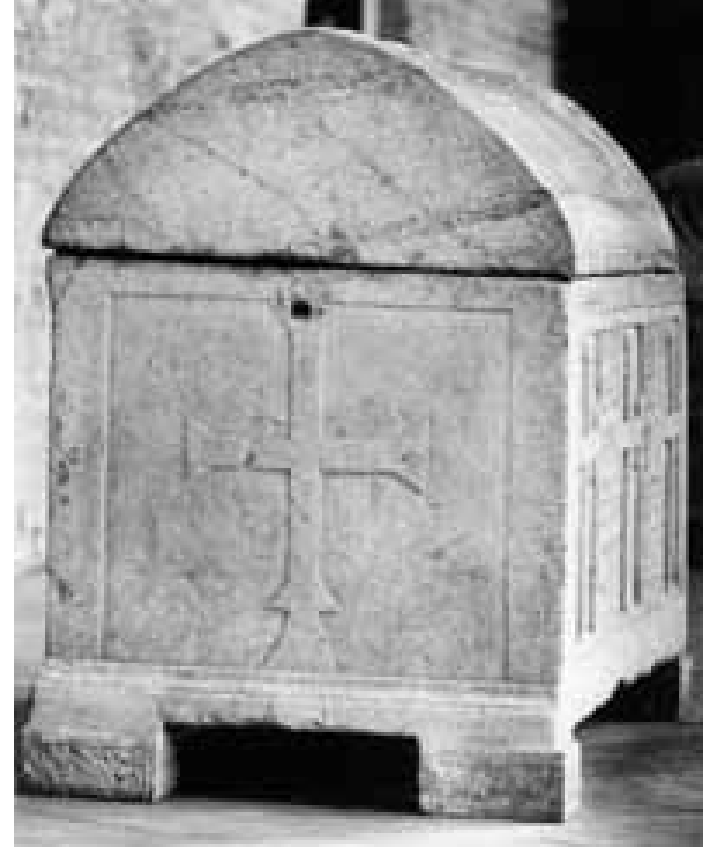

26 Classe, S. Apollinare: attuale fianco sinistro della cassa del sarcofago dell'arcivescovo Grazioso, della metà circa del VI sec.

157 R. Venuti, Antiqua numismata II (Roma 1744) fig. a 102. Poi in Anonimo, Rovine dell'antica Roma e del vicinato (Roma s. d. [XVIII sec.]) tav. 37, e in R. Venuti, Accurata e succinta descrizione topografica delle antichità di Roma I (Roma 1803) 118-119 tav. 22 tra le 118-119.

158 Anonimo, Varie vedute di Roma antica e moderna (Roma 1748) tav. 38. Mostra il lato ovest, faccia verso est, dove son indicati 8 lastre e 8 pilastrini tutti uguali e sormontati da un disco, divisi 5 a sinistra (sud), il centrale, 2 a destra (nord) (di cui uno occupato dal quadrato della torre).

159 A. Robinson, Vedute di Roma, in: Aa. Vv., Piranesi. Incisioni - rami - legature - architetture (1978) 49 fig. 251. Mostra il lato ovest, faccia verso ovest, con 13 pilastrini e 11 lastre $(5+1+5)$ : si distingue chiaramente la forma della terminazione superiore dei pilastrini, o a disco o a pigna-pinnacolo.

160 Anonimo, Nuova raccolta di cento principali vedute antiche e moderne dell'alma città di Roma e delle sue vicinanze (Roma 1796) tav. 44. Mostra il lato ovest, faccia verso ovest, con 5 lastre e 5 pilastrini a nord, la lastra centrale con 2 pilastrini più alti, e 6 lastre e 5 pilastrini a sud.

${ }^{161}$ I disegni preparatorii sono nella Biblioteca Vaticana, Cod. Vat. lat. 9839, I, foll. 5v e 6r e nel Cod. Vat. lat. 13479, I, foll. 128-132, e sono stati analizzati e valorizzati da A. Guiglia Guidobaldi in: Christiana loca (nota 16) 1495. 1497-1498 fig. 6. Le incisioni in G. B. L. G. Séroux d'Agincourt, Storia dell'arte col mezzo dei monumenti dalla sua decadenza nel IV secolo fino al suo risorgimento nel XVI, I (Milano 1824) 48; II (1825) 26-27 tav. 19 n. 3 (lato est, faccia est); n. 4 (lato ovest, faccia est); n. 5 (lato est, faccia ovest); n. 6 (lato ovest, faccia est).

162 A. Uggerl, Vues des édifices de Rome antique dans l’intérieur III 2 (Roma 1810) tav. n. 58. Mostra parapetti solo nella parte occupata dalla torre.

163 G. B. Cipriani, Degli edifici antichi e moderni di Roma. Vedute in contorno II (Roma 1817) tav. 12. Stesso taglio della stampa di Uggeri, e pure qui solo parapetti (4, con 5 pilastrini) in corrispondenza della torre.

${ }^{164}$ M. Dubourg, Views of the Remains of Ancient Building in Rome, and its Vicinity (London 1844) tav. 24. Riprende esattamente la stampa di Venuti (nota 157:1803).

${ }^{165}$ L. Rossini, Le antichità romane (Roma 1829) tav. 23 (lato ovest, faccia verso ovest, con soli 5 pilastrini e 3 lastre, in corrispondenza della torre).

166 P. Colini Lombardi, Ponte Salario attraverso la storia, Capitolium 7, 1931, 394 fig. a p. 393. Ripropone un taglio simile a quello del Piranesi, con identico numero di pilastrini (23) e di lastre (11) nel lato ovest, faccia verso ovest.

167 A. Nibby, Analisi storico-topografico-antiquaria della carta de’ dintorni di Roma II (Roma 1837) 594.

168 G. Avakian, Rilievi inediti di monumenti etruschi e romani, Ephemeris Dacoromana 6, 1935, 152-162 fig. 10 in alto (lato est, faccia est) e in basso (lato est, faccia ovest). Il disegno delle lastre e dei pilastrini (fig. 10 in basso) è ripreso in A. Guiglia Guidobaldi in: Ecclesiae Urbis (nota 6) 1495-1496. 1499-1500 fig. 7.

${ }^{169}$ Che ci permette di analizzare 16 lastre (14 + le due centrali con le epigrafi) e 18 pilastrini (14 + i quattro centrali).

${ }^{170}$ C. Barsanti - A. Guiglia Guidobaldi in: Guidobaldi - Barsanti - Guiglia Guidobaldi (nota 3) 263. 

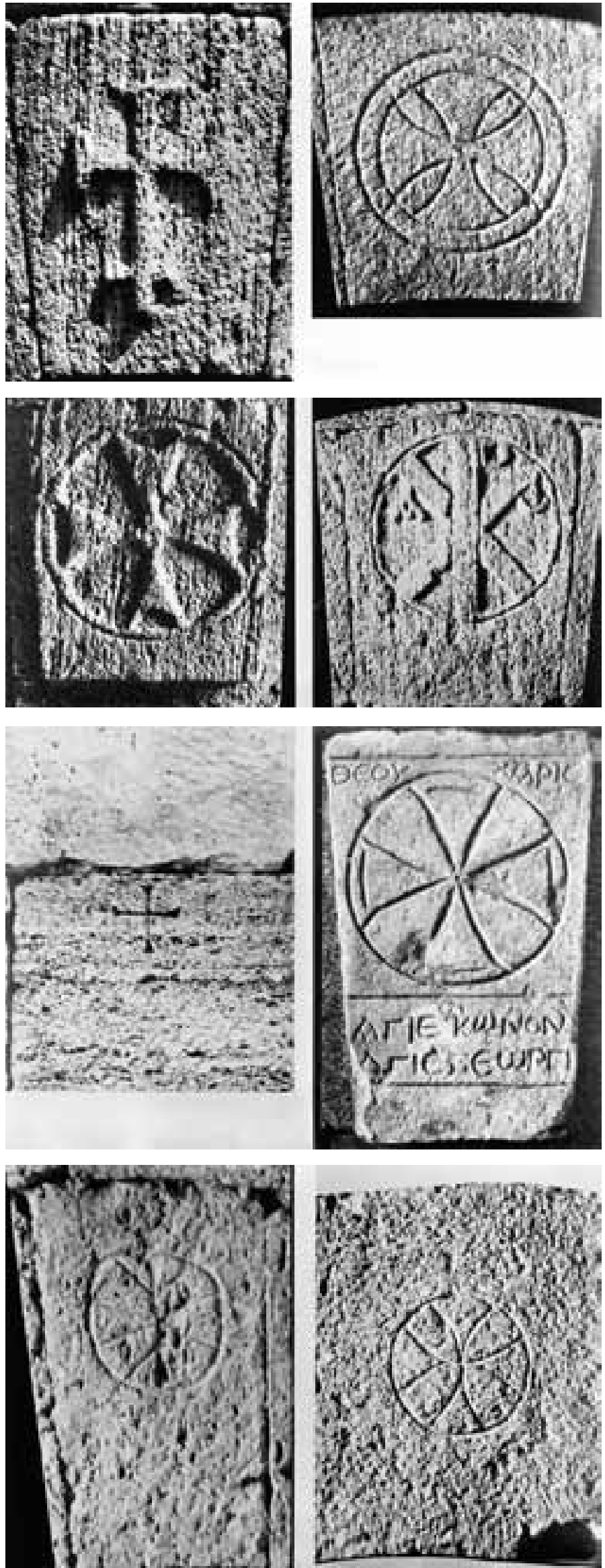

27 Roma, porta Pinciana: decorazioni sull'arco interno e sull'arco esterno

28 Roma, porta Latina: decorazioni sull'arco interno e sull'arco esterno

29 Roma, porta Appia: decorazioni alla base della torre orientale e sull'arco interno

30 Roma, porta Ostiense: decorazioni sull'arco interno e sull'arco esterno 


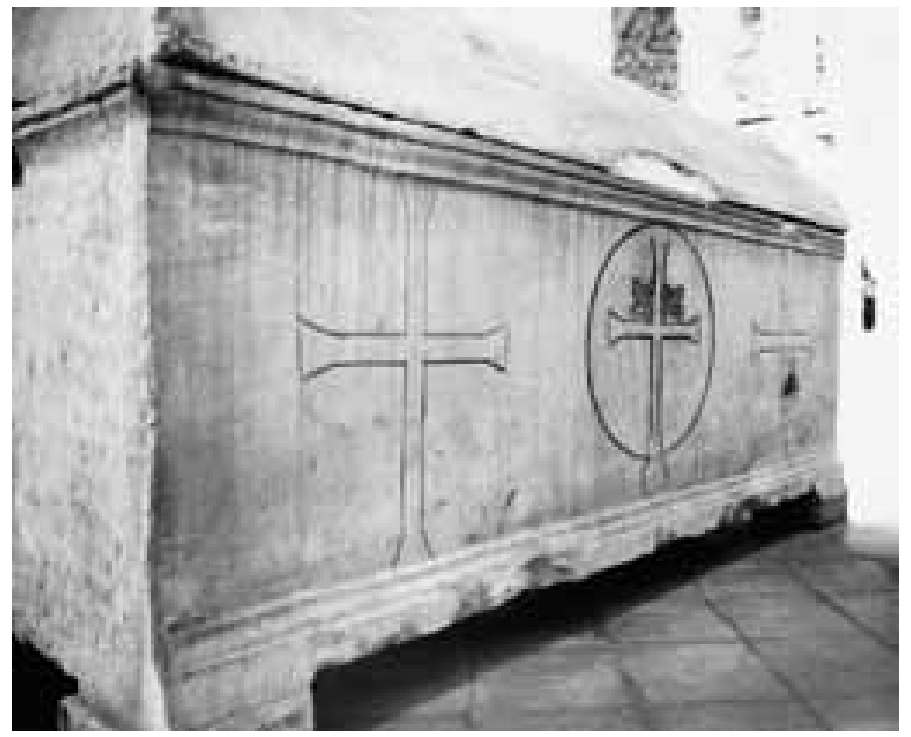

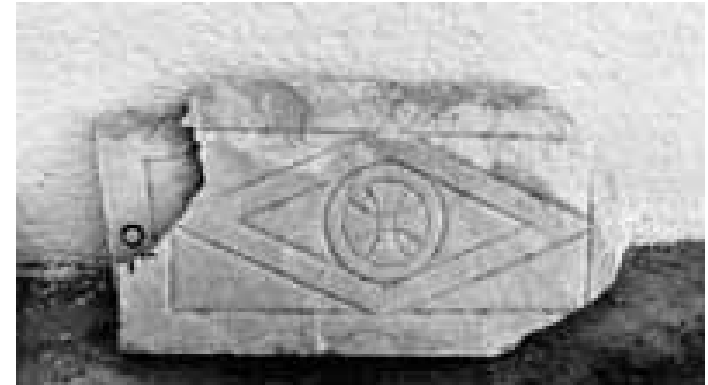

32 Mileto, Museo: lastra a incisione, inv. 70

31 Classe, S. Apollinare: attuale retro della cassa del sarcofago dell'arcivescovo Grazioso, della metà circa del VI sec.

produzione di Costantinopoli e della sua area, con il rombo centrale contenente un disco e due dischi laterali ad esso tangenti: si ha tutta l'impressione d'esser davanti a una creazione sul posto di maestranze grecocostantinopolitane, al modo dello schema tripartito con rombi di S. Clemente. È un'impressione che trova una clamorosa conferma dal disegno del terzo pluteo a partire da sinistra nel lato ovest, faccia verso est, come ce lo propone il Cod. Vat. lat. 1379, fol. 130 (fig. 37), dove entro il rombo centrale vediamo un piccolo fiore a 6 petali e nel disco ai suoi lati le lettere greche $\mathrm{H}$ e $\Gamma$, e nel pilastrino a sinistra dell'epigrafe nel lato est, faccia verso ovest, la lettera greca $\Gamma^{171}$ : se non posson esser prova d'importazione dei manufatti, sono comunque segno incontrovertibile della mano d'artefici greco-costantinopolitani, e della medesima origine di quelli delle decorazioni delle porte urbiche, dato che in 7 dei dischi nei plutei e in 4 dei dischi nei pilastrini noi vediamo croci della stessa foggia di quelle delle porte, come ha già osservato G. B. Giovenale ${ }^{172}$, e in particolar modo della croce entro clipeo di porta Pinciana (fig. 27). Tre lastre presentano, per la loro lunghezza inferiore, un solo rombo a occupare l'intero campo interno, o da soli (due volte) o con un disco contenente un fiore a 6 petali. Ma le sorprese non sono finite: se con tale motivo siam nel medesimo àmbito culturale delle prime lastre, con altre due avvertiamo anche l'opera di maestranze romane, per via del motivo a pelte in una e ancor più di quello a cancello nell'altra ${ }^{173}$. Opera rafforzata dalla considerazione di altre 4 lastre senza decorazioni. Dunque nei parapetti del ponte il tema della varietas è declinato non soltanto con la diversità degli schemi, ma pure con la compresenza di maestranze d'origine e di cultura difforme ${ }^{174}$. E che si debba riconoscer ancor una volta l'acume di Raffaele Cattaneo, quando nel 1888 parlava dei parapetti come opera di »scalpelli greci $\aleph^{175}$ - sia pur integrando il suo giudizio con il riconoscimento della collaborazione di artefici romani -, e non si possa condivider il giudizio di recente proposto dell'impresa d'una bottega locale ${ }^{176}$,

${ }^{171}$ Come ha ben visto A. Guiglia Guidobaldi in: Ecclesiae Urbis (nota 6) 1498, la quale ha però lasciato cadere l'osservazione, che andava contro la sua teoria d'un'esecuzione da parte di bottega romana e di una massima limitazione della presenza di maestranze greco-costantinopolitane a Roma.

172 Giovenale (nota 137) 258 fig. 49.

173 Russo (nota 1) 23; A. Guiglia Guidobaldi in: Ecclesiae Urbis (nota 6) 1500.

${ }^{174}$ Penso che sia una soluzione preferibile a quella di vedere, stante l'unitarietà (non l'omogeneità) dell'insieme, l'opera di maestranze greco-costantinopolitane che riprendono schemi consueti a Roma, dunque con un altro segno dell'interazione uomo - ambiente: la soluzione proposta va nella direzione d'una maggiore integrazione dell'elemento romano, dato che alla realizzazione delle decorazioni sarebbero associate maestranze non greco-costantinopolitane.

175 R. Cattaneo, L'architettura in Italia dal secolo VI al mille circa (Venezia 1888/89) 34 (ed. francese 1890, p. 40). Rettifico dunque quanto avevo scritto in Russo (nota 1) 23 nota 44.

176 A. Guiglia Guidobaldi in: Christiana loca (nota 16) 269; eadem in: Ecclesiae Urbis (nota 6) 1491. Il giudizio corretto di Broccoli (nota 69) 137 (»uno fra i migliori esempi della scultura di ambiente bizantino a Roma«), è invece dovuto a un fraintendimento (cf. A. Guiglia Guidobaldi in: Ecclesiae Urbis [nota 6] 1496 nota 41). 


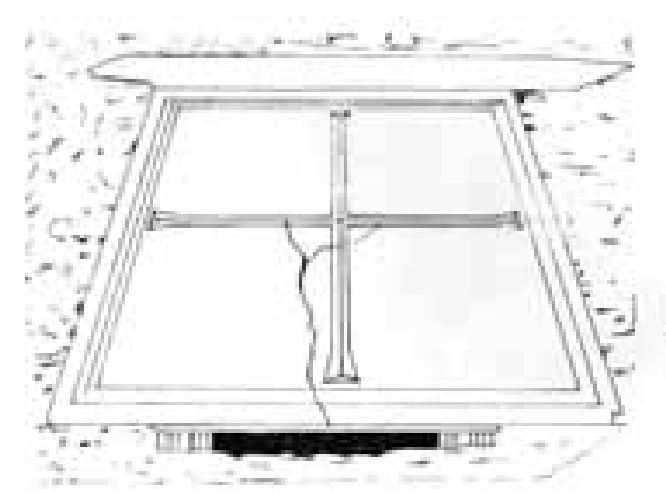

33 Roma, Ss. Apostoli: ricostruzione della mensa dell'altare del VI sec. è provato anche dalla considerazione dei pilastrini. Non son i motivi a riquadrature ${ }^{177}$ e specchiature dei corpi ${ }^{178}$ che caratterizzano i pilastrini, bensì la terminazione superiore, a pomello tipo pigna o pinnacolo o a disco indipendente. I quattro esemplari a pomello trovan confronti in una vasta area che va da Ravenna a Grado, dalla Grecia alla Palestina e alla Siria ${ }^{179}$. Sconcertanti i pilastrini con disco indipendente $^{180}$, che non paiono trovar un preciso corrispettivo nemmeno in Oriente, giacché l'unico esemplare indicato da A. Guiglia, della chiesa di Apa Jeremias a Saqqara (fig. 41) ${ }^{181}$, non mostra segni d'incasso per le lastre sui due lati, è privo di decorazioni nel corpo ed è di proporzioni tali che, in assenza a mia conoscenza di plutei di recinzione (di contro ai molti capitelli) nell'area del monastero, e per via di due spalliere di cattedra (fig. 42) ${ }^{182}$ - e quasi sicuramente tre ${ }^{183}$ - a disco indipendente superiore, anche il pezzo ritenuto pilastrino sembra piuttosto parte di spalliera di cattedra. Ancora una creazione originale delle maestranze greco-costantinopolitane a Roma, che paiono aver accentuato lo stacco del disco rispetto al corpo da manufatti come la parte tergale della cattedra della chiesa di Bettir in Siria, del 469 circa $^{184}$, alla maniera degli artefici delle cattedre di Saqqara, dato che un pilastrino della chiesa di Bāfetīn, del VI secolo ${ }^{185}$, ha sì un disco in alto, ma sempre nel corpo a parallelepipedo e sormontato da un pomello. La Guiglia ha già indicato per altri motivi presenti nella decorazione del ponte, come il fiore a 6 petali entro piccolo disco al centro di una delle lastre o il triplice intreccio di 8 con tondo centrale nel disco di uno dei pilastrini, confronti con opere greco-costantinopolitane ${ }^{186}$, su cui si può senz'altro convenire, ed è pertanto ancor più strano che per

177 S’è conservata la fotografia di uno dei pilastrini con le riquadrature in una foto di H. Parker (L. Chiumenti - F. Bilancia, La campagna romana VI [sulla base degli appunti lasciati da G. e F. Tomassetti] [1977] fig. a p. 154; A. Guiglia Guidobaldi in: Ecclesiae Urbis [nota 6] fig. 4 a p. 1492), in orizzontale tra due pilastrini con terminazione a disco indipendente. La fotografia riprende il lato est del ponte distrutto. Un'altra fotografia di Parker, con l'altro lato del ponte (B. Brizzi, Roma cento anni fa nelle fotografie della raccolta Parker [1975] fig. a p. 213) ci mostra la base quadrangolare del pilastrino.

${ }^{178}$ I corpi di due pilastrini con terminazione a pomello eran privi d'incorniciatura e di decorazioni.

${ }^{179}$ Ma senza dimenticare i frammenti di due pilastrini di Roma, l'uno nel coemeterium maius (Broccoli [nota 69] 202 n. 150 tav. 41), l'altro nel cimitero di S. Ippolito (ibidem 268 n. 289 tav. 70).

${ }^{180}$ La Guiglia ha messo in rilievo che due con il disco indipendente sono visibili chiaramente (di uno dei due sopra il pelo dell'acqua spunta solo la parte superiore del disco) nella fotografia Parker più nota (A. Guiglia Guidobaldi in: Ecclesiae Urbis [nota 6] 1492-1493). In realtà il pilastrino ch'emerge dall’acqua è ben visibile anche nell’altra foto Parker (Brizzi [nota 177] fig. a p. 213).

${ }^{181}$ La Guiglia non s’è accorta che il pezzo da lei pubblicato (A. Guiglia Guidobaldi in: Ecclesiae Urbis [nota 6] 1502 fig. 9) è il medesimo pubblicato da J. E. Quibell, Excavations at Saqqara (1907-1908) (1909) 97 tav. 3 in basso a sinistra, e che questo non è »un esemplare simile, un tempo in opera nella chiesa principale del convento« (A. Guiglia Guidobaldi in: Ecclesiae Urbis [nota 6] 1502 nota 49).

182 J. E. Quibell, Excavations at Saqqara (1908-09, 1909-1910). The Monastery of Apa Jeremias (1912) 131 tav. 6, 4 (cattedra con croce greca nello schienale in alto del tutto simile a quella del pezzo precedente, nel cortile »degli ottagoni«); 133 tav. 14 (schienale di cattedra con 6 gradini: nel disco, entro un clipeo con epigrafe menzionante il Padre il Figlio lo Spirito Santo, una conchiglia). La cattedra con 6 gradini è oggi nel Museo Copto del Cairo: G. Duthuit, La sculpture copte (1931) 46 tav. 39 (con datazione al V sec.).

${ }^{183}$ Quibell (nota 181) 108 tav. 38, 5 a destra (disco con piccola porzione di spalliera: nel clipeo croce greca e tra i bracci la metà d'un fiore complesso che completo conterrebbe $3 \times 4=12$ petali, dipartentisi a gruppi di 3 da una corolla centrale, dunque tra $\mathrm{i}$ bracci $3+1$ e $1 / 2+1$ e $1 / 2$ petali). Potrebbe forse esser una testata di spalliera di cattedra anche il manufatto ad arco oltrepassato con croce entro due rami a corona, legati in basso, di Ossirinco (E. Breccia, Municipalité d’Alexandrie. Le Musée gréco-romain, 1925-1931 [1932] tav. 47 fig. 166), che la Krumeich (nota 48) I, 83. 133-134 e II, 124 n. N-24 tav. 121, dice »Bogenfeld«.

184 Tchalenko (nota 113) 177-178 tav. 461 fig. 320 in alto.

185 Tchalenko (nota 113) 141-142 tav. 369 in basso a destra, fig. 224.

${ }^{186}$ Per il piccolo fiore a 6 petali al centro di un disco, un pluteo di S. Sofia di Costantinopoli (A. Guiglia Guidobaldi in: Ecclesiae Urbis [nota 6] 1500-1501 fig. 8). Per il triplice intreccio di 8 rimando a uno studio di Barsanti (nota 90) 9-16 figg. 1, 7-13, che invero indaga un'area più vasta comprendente pure Palestina e Siria: per quest'ultima occorre aggiunger l'architrave del portale nord della chiesa di Deir Sētā (Naccache [nota 130] II tavv. 34, 2 e 279, 2) e l'architrave del portale ovest del battistero della 
il ponte Salario accetti ${ }^{187}$ il mio giudizio del 1979 quando vi vedevo il prodotto di maestranze romane, »senza peraltro poter escludere del tutto l'intervento o la supervisione di qualche artefice non romano «188: a distanza di 25 anni da quel saggio pionieristico, oggi nella fattispecie ritengo che la valutazione si debba rovesciare, e si debba concluder che le decorazioni del ponte son frutto del lavoro in situ di maestranze greco-costantinopolitane, che hanno fatto realizzare una parte dell'opera ad artefici romani loro associati nell'impresa. Un'impresa che dall'attento esame della documentazione rivela un'esecuzione coe-
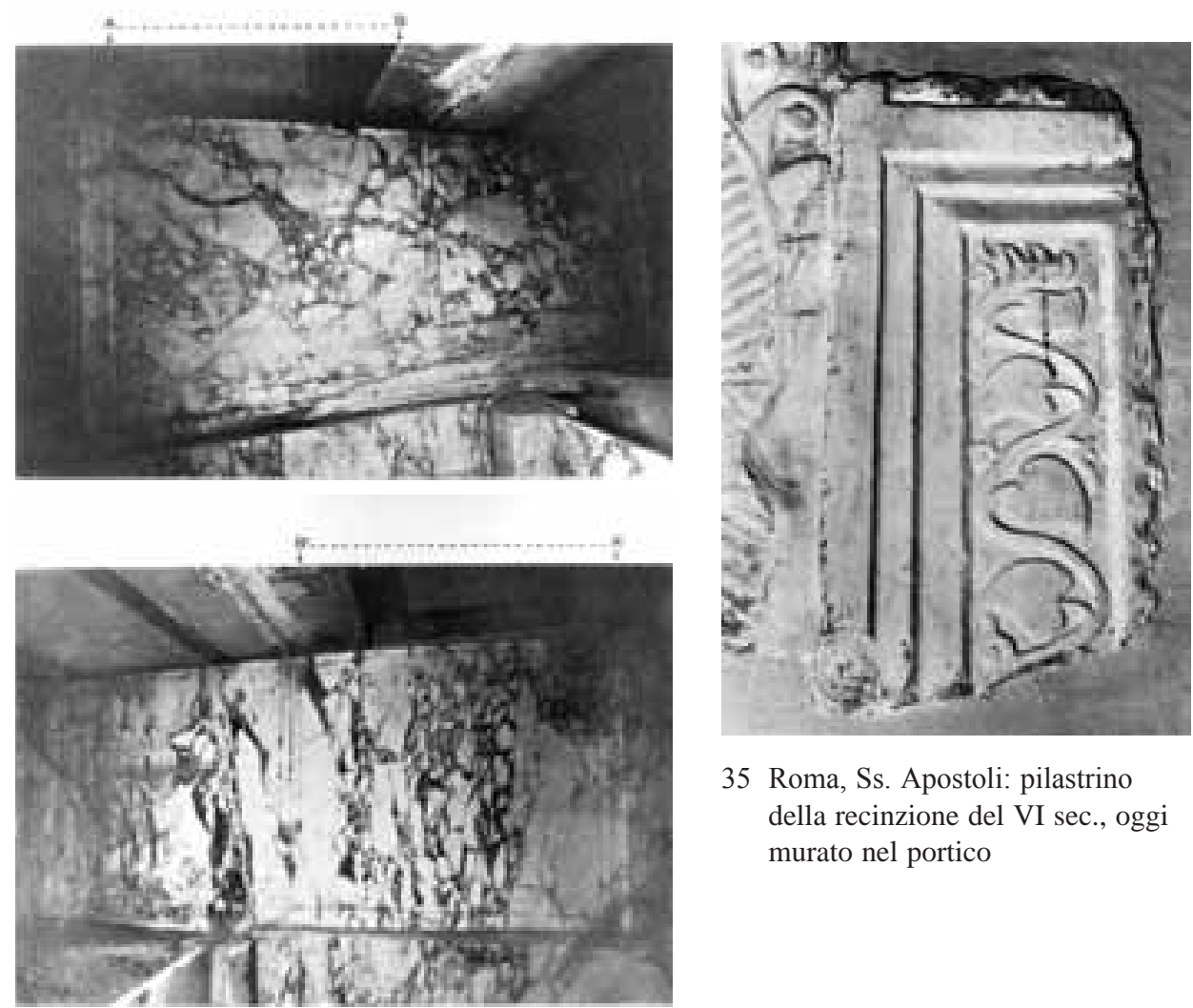

35 Roma, Ss. Apostoli: pilastrino della recinzione del VI sec., oggi murato nel portico
34 Roma, Ss. Apostoli: i due pezzi distinti di ricupero di marmo pavonazzetto, costituenti la mensa dell'altare del VI sec. va e unitaria年, sia pur non omogenea, senza le incertezze verso un presunto reimpiego di pezzi più antichi mostrate dalla critica di ieri e di oggi ${ }^{190}$. E il rinnovato esame mi porta a rivedere anche la valutazione che ne diedi nel 1979, a proposito delle decorazioni delle lastre con schema tripartito, che oggi mi appare una sfida 'difficile' per l'equilibrio della composizione nell'accostamento di due dischi a un rombo centrale, ma in cui prevale l'elemento dell'originalità - come per la scelta della terminazione a disco indipendente nella maggior parte dei pilastrini - anche a caratterizzare il livello delle maestranze (che, si è visto, sono non di Costantinopoli tout court, ma greco-costantinopolitane); e oggi mi convince pure che ai lati delle due epigrafi sian stati posti due pilastrini di dimensioni maggiori e recanti nel disco una croce, a far da pendant alle croci sopra e ai lati delle porte urbiche. Ma ribadisco come nel 1979 che per un ponte nel 565 una tale decorazione, sia pur mancante entro le incorniciature di alcune lastre, è »un vero >lusso «, dati i tempi « ${ }^{191}$ e la prova d’uno sforzo considerevole compiuto per 'monumentalizzare' uno degli accessi chiave a Roma dal nord, dunque adoperando alcune delle maestranze migliori tra quelle greco-costantinopolitane allora presenti in città, cui affiancare collaboratori romani all'altezza del compito assegnato.

L'opera di maestranze greco-costantinopolitane e di maestranze romane si ritrova egualmente nel complesso pelagiano di S. Lorenzo f. l. m. $(579-590)^{192}$, ma in combinazioni sensibilmente variate, accanto alla

chiesa dei Ss. Paolo e Mosè (515) di Darqīta (ibidem tav. 305, 2). Per fiori multipetali, girandole, etc. la Guiglia Guidobaldi op. cit. 1501-1502, cita un repertorio soprattutto siriaco.

187 A. Guiglia Guidobaldi in: Ecclesiae Urbis (nota 6) 1496.

188 Russo (nota 1) 23.

189 In tal senso già Russo (nota 1) 23.

190 Kautzsch (nota 64) 52; A. Guiglia Guidobaldi in: Ecclesiae Urbis (nota 6) 1500.

191 Russo (nota 1) 23.

192 La Melucco Vaccaro (nota 3:1999) 99, parla di »ricorso alla produzione bizantina«; A. Guiglia Guidobaldi in: Christiana loca (nota 16) 269 e eadem in: Ecclesiae Urbis (nota 6) 1505, di una bottega romana. 

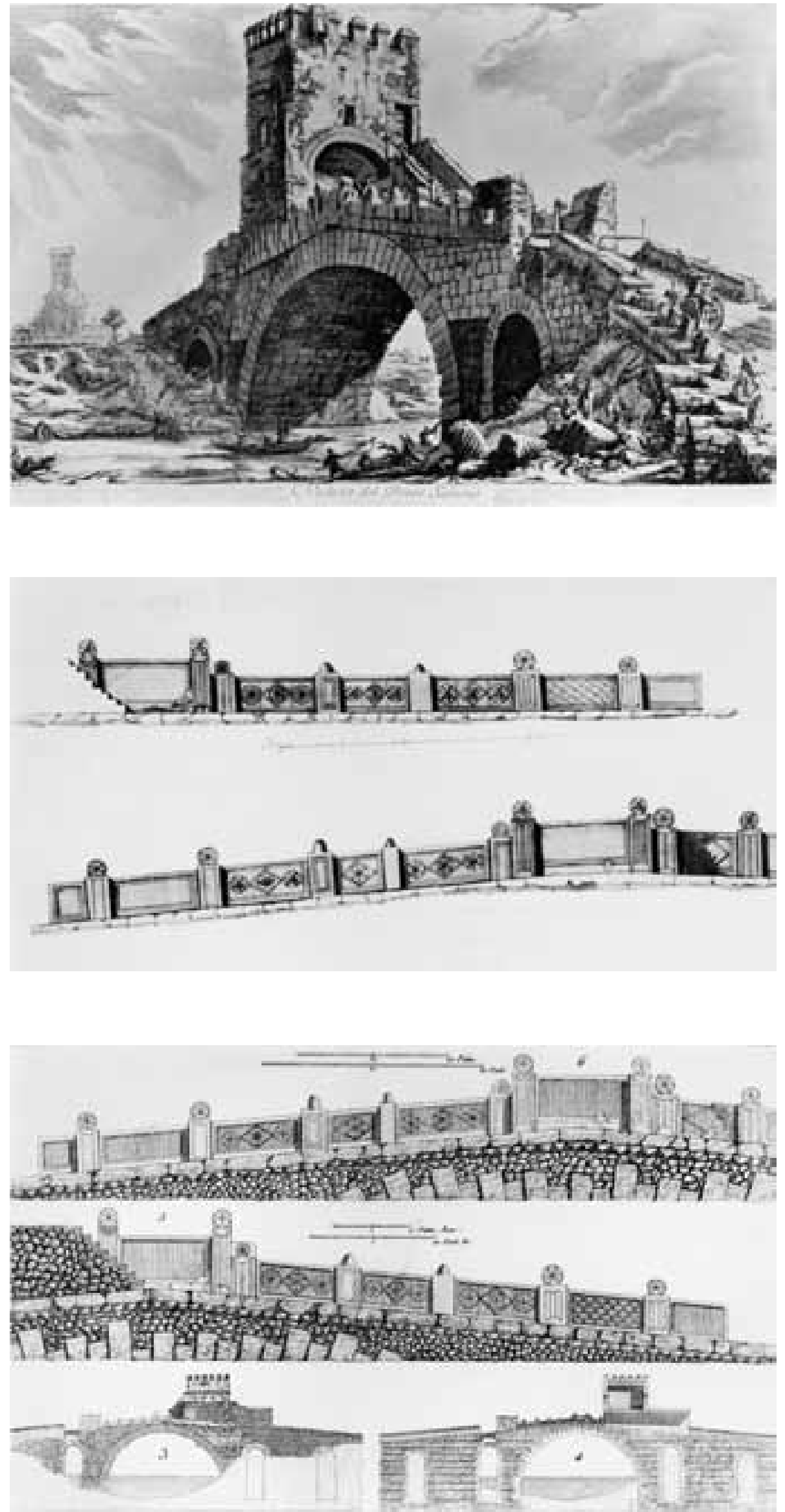

36 Roma, ponte Salario sull'Aniene: lato ovest, faccia verso ovest (incisione di $\mathrm{G}$. B. Piranesi, 1754)

37 Roma, ponte Salario sull'Aniene: lato ovest, faccia verso est (disegno conservato nel Cod. Vat. lat. 1379, fol. 130)

38 Roma, ponte Salario sull'Aniene: lato est, facce est e ovest, e lato ovest, faccia est 
quantità di materiale classico reimpiegato ${ }^{193}$. Ho riesaminato il frammento di pluteo ${ }^{194}$ con croce entro un rombo, di cui trattai nel $1979^{195}$, giacché C. Barsanti lo ha decisamente collegato cronologicamente con le lastre di S. Clemente, anche con l'epoca di Giovanni I (523-526) per via d'una donazione del pontefice all'edificio precedente ${ }^{196}$; ma son rimasto convinto che il pezzo sia posteriore alla recinzione di S. Clemente, come allora resto incerto tra una produzione a Roma da parte di maestranze grecocostantinopolitane o un'importazione del pezzo già rifinito ${ }^{197}$, e come allora propendo di più per l'importazione della lastra (e dunque di tutte le lastre della recinzione, purtroppo perdute) già rifinita ${ }^{198}$. Ho riesaminato pure i due dadi nella galleria centrale sopra il nartece $^{199}$ e i due plinti delle ultime colonne verso l'abside

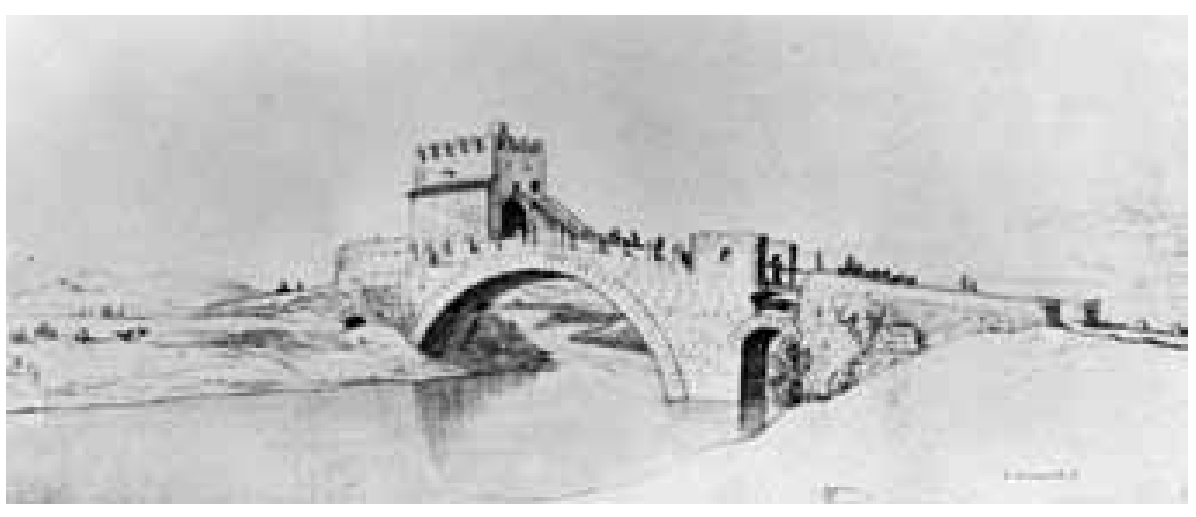

39 Roma, ponte Salario sull'Aniene: lato ovest, faccia verso ovest (disegno di F. Caracciolo, 1826)

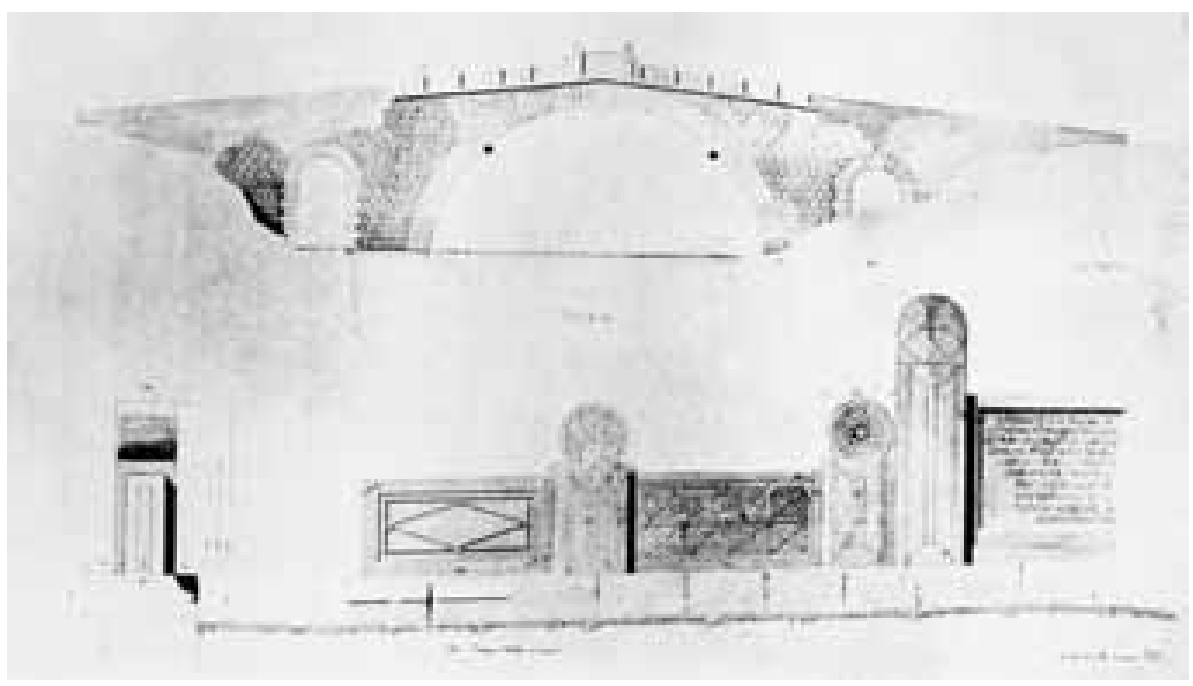

40 Roma, ponte Salario sull'Aniene: lato est, faccia est e faccia ovest

193 Per un primo orientamento sul materiale classico reimpiegato vd. Russo (nota 1) 24; S. Ciranna, Spolia e caratteristiche del reimpiego nella basilica di San Lorenzo fuori le mura a Roma (2000) 83-118 figg. 40-90; P. Fancelli, Gli spolia tra architettura e restauro nelle chiese di Roma, in: Ecclesiae Urbis (nota 6) 843-858; S. Ciranna, La lettura architettonica degli spolia nelle chiese di Roma, in: Ecclesiae Urbis (nota 6) 859-874.

194 Non di ambone come immagina Broccoli (nota 69) 215-220 n. 158 tav. 45 (con datazione confusa »Metà del secolo VI [523-526?; 579-590?]«); U. Broccoli, Sculture altomedievali del chiostro di San Lorenzo f.1.m., RACr 58, 1982, 295-297 fig. 5.

195 Russo (nota 1) 24 fig. 8.

196 C. Barsanti in: Guidobaldi - Barsanti - Guiglia Guidobaldi (nota 3) 161-163 fig. 246.

197 Lo dissi chiaramente allora: »presenza di maestranze e/o prodotti greco-cost/i« (Russo [nota 1] 24).

198 Russo (nota 1) 24: »La resa qualitativa deve far considerare con grande attenzione tale frammento nell'ambito della coeva produzione greco-cost/a.« Non pare aver compreso il punto, anche lì dove pongo il problema d'»una sorta di stasi inventiva nella produzione delle botteghe e/o maestranze greco-cost/e«, lasciando cioè sempre aperte le due possibilità, di maestranze straniere attive a Roma o di prodotti importati già rifiniti, C. Barsanti in: Guidobaldi - Barsanti - Guiglia Guidobaldi (nota 3) 161, se interpreta il mio pensiero tout court come rinvio all'attività di maestranze straniere a Roma e dichiara »criptico« il mio rinvio alla coeva produzione greco-cost/a. Sono invece completamente d'accordo con C. Barsanti op. cit. 163 nota 207, quando viene stigmatizzata l'attribuzione di Broccoli (nota 69) 213-215. 220-221 nn. 157. 159-162 tavv. 44 e 46, di lastre classiche di rivestimento parietale al momento della costruzione pelagiana di S. Lorenzo: invece esse furon »reimpiegate nella chiesa del VI secolo insieme ad analoghe paraste corinzie« (C. Barsanti in: Guidobaldi - Barsanti - Guiglia Guidobaldi [nota 3] 163 nota 207). Il Broccoli (nota 194) 294-295 fig. 4, ha insistito sul punto.

199 Russo (nota 1) 24-29 figg. 9-14 (con la bibl. precedente); Broccoli (nota 69) 227-232 nn. 172-173 tavv. 51-54; A. Guiglia Guidobaldi in: Ecclesiae Urbis (nota 6) 1509-1511 fig. 16. 


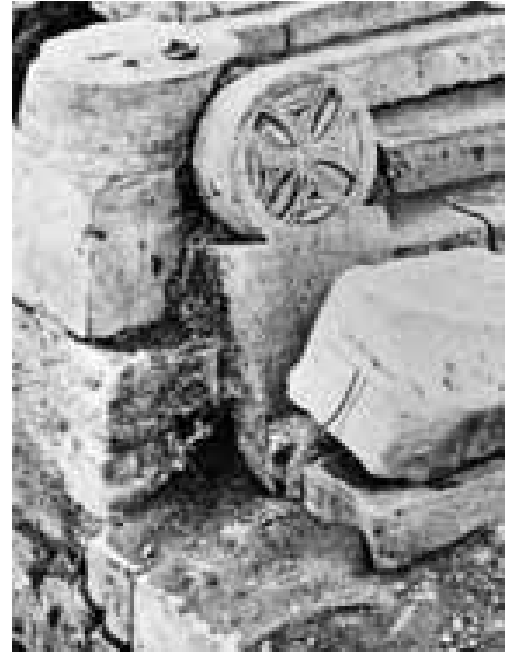

41 Saqqara, Apa Jeremias: spalliera (?) di cattedra
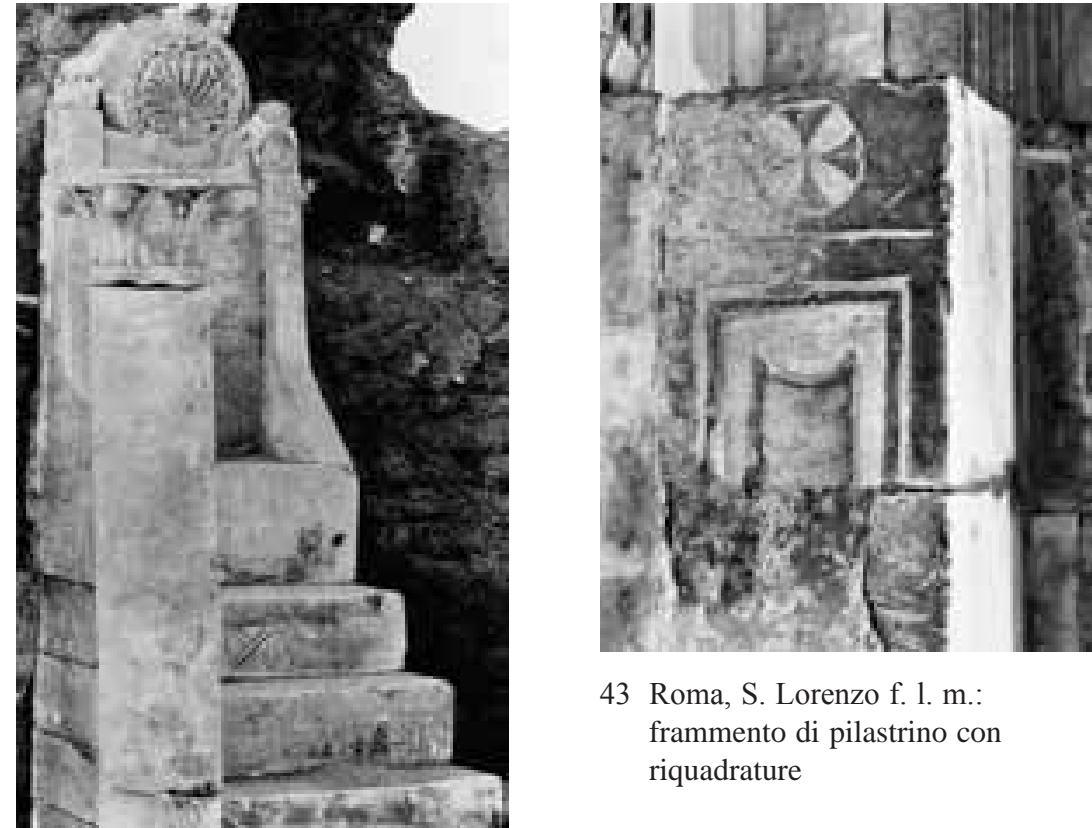

43 Roma, S. Lorenzo f. 1. m.: frammento di pilastrino con riquadrature
42 Saqqara, Apa Jeremias: spalliera di cattedra con 6 gradini (oggi al Cairo, Museo Copto)

pelagiana ${ }^{200}$, e mi son riconfermato su quanto scrissi nel 1979, vale a dire che nei dadi noi assistiamo all'opera »di maestranze locali che imitano ora decisamente gli esiti stilistici« dei prodotti greco-costantinopolitani, e con il dado destro ch'»è di fattura superiore al sinistro«, mentre nei plinti siam in presenza di manufatti di »romani che non imitano o non desiderano imitare un modello preciso«, ma »seguono soprattutto il proprio senso compositivo « ${ }^{201}$. Avevo accostato i due dadi a prodotti coevi di Ravenna, e altrettanto ha fatto il Broccoli; la Melucco Vaccaro, che li ritiene "produzione bizantina« »e la cui area di distribuzione coincide con le zone costiere dell'impero bizantino«, li accosta a lastre di S. Eufemia a Grado, del tempo del patriarca Elia $(571-587)^{202}$, e la Guiglia ha raccolto l'indicazione, ampliandola ad altre sculture di Grado e di Parenzo ${ }^{203}$. Ma le affinità tra i dadi di S. Lorenzo e sculture di Ravenna, Grado e Parenzo son dovute non all'importazione di tali pezzi dalle »zone costiere dell'impero bizantino« come opina A. Melucco Vaccaro, bensì al fatto che a Roma, a Ravenna, a Grado, a Parenzo siamo davanti a maestranze locali che imitano i prodotti greco-costantinopolitani. Oggi io penso per i dadi di S. Lorenzo che non si possa nemmeno escluder la presenza come suggeritore e/o rifinitore di qualche artefice della maestranza del ponte Salario rimasto sul posto e organizzatore o partecipe della bottega del cantiere pelagiano.

Una considerazione che si è riproposta nella valutazione dei pilastrini superstiti della recinzione ${ }^{204}$. Infatti i due con riquadrature (figg. 43. 44) mostrano nella delineazione delle riquadrature una sensibilità formale

${ }^{200}$ Russo (nota 1) 24-25. 29-31 figg. 15-16 (con la bibl. precedente); Broccoli (nota 69) 232-234 nn. 174-175 tav. 55; A. Guiglia Guidobaldi in: Ecclesiae Urbis (nota 6) 1508-1509 fig. 15.

201 A. Guiglia Guidobaldi in: Ecclesiae Urbis (nota 6) 1509, non ha raccolto questa mia distinzione tra dadi e plinti, e si limita a riconoscer che i dadi sono »Di livello più elevato«.

202 Melucco Vaccaro (nota 3:1999) 99.

${ }^{203}$ A. Guiglia Guidobaldi in: Christiana loca (nota 16) 269; A. Guiglia Guidobaldi in: Ecclesiae Urbis (nota 6) 1510-1511. Sulle sculture di Grado e Parenzo vd. Russo (nota 9) 177-179 nn. 123-124 figg. 149-150 (con la bibl. precedente); A. Guiglia Guidobaldi in: Ecclesiae Urbis (nota 6) 1510-1511 figg. 17-19.

204 1) chiostro, $1^{\circ}$ piano, lato sud, reimpiegato in una finestra: F. Mazzanti, La scultura ornamentale romana nei bassi tempi, Archivio storico dell'arte serie seconda 2 (1896) 52; Broccoli (nota 69) 222-223 n. 165 tav. 48 (Broccoli lo dice »completo«, ma nel reimpiego è stato privato in verticale della fascia esterna della riquadratura, come ho verificato sul posto); Broccoli (nota 194) 297 fig. 6; A. Guiglia Guidobaldi in: Ecclesiae Urbis (nota 6) 1503-1504 fig. 10. 2) chiesa, ambone di destra, reimpiegato nei gradini della scala: $\mathrm{cm} .30 \mathrm{~h} \times 22$ lato decorato $\times 23$ : Mazzanti op. cit. 52; Broccoli (nota 69) 223 n. 166 tav. 48.3 ) chiostro, $1^{\circ}$ 

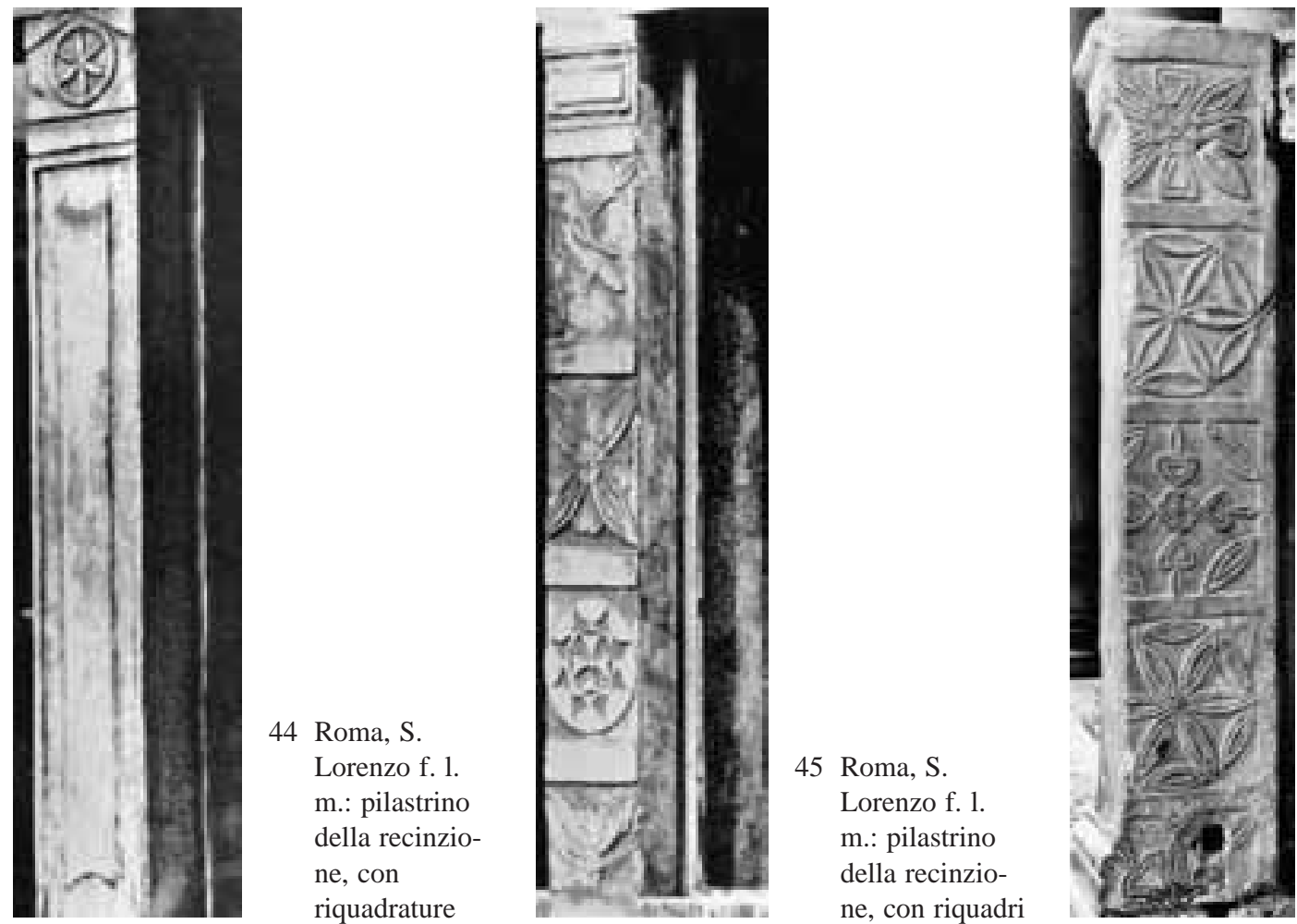

46 Roma, S.

Lorenzo f. 1 .

m.: pilastrino

della recinzione, con riquadri

superiore a quella ch'è riscontrabile nei due con riquadri (figg. 45. 46); e d'altra parte questi ultimi nella loro partizione non trovan riscontri a Roma bensì a Costantinopoli, come ha indicato A. Guiglia, che ha pensato per tale decorazione di S. Lorenzo »ad un suggerimento di matrice bizantina ${ }^{205}$. Conviene esaminare con attenzione i pilastrini. Quello reimpiegato nell'ambone sinistro (fig. 46) è decorato non soltanto nel lato est, ma anche nel lato nord, e ha visto bene F. Mazzanti nel riprodurlo capovolto rispetto alla posizione odierna, in quanto nel lato nord vi era la riquadratura con cornice e l'incasso per la lastra, e in basso (l'alto originario) resta un riquadro decorato con lo stesso motivo che compare nella faccia visibile nel riquadro oggi in alto (in origine in basso $)^{206}$. Nel reimpiego medievale il pezzo è stato capovolto, ma è stata sfruttata nel lato nord la parte con l'incasso ${ }^{207}$ per inserirvi la lastra dell'ambone. Ebbene nel lato nord l'articolato sistema di riquadrature oggi in basso (in origine in alto) è sì corrispondente con un listello della faccia est, quello visibile in basso, ma corrisponde appunto a una semplice fascia piatta, peraltro nemmeno ben levigata. Ferma restando la paternità romana, di maestranze d'imitazione dei prodotti greco-costantinopolitani, per la faccia est, le riquadrature della faccia nord potrebbero anch'esser assegnate a una mano greco-costantinopolitana, la stessa che potrebbe aver suggerito lo schema decorativo. Ma se è valida questa conclusione, ne viene che comunque c'era commistione di mani, dato che il riquadro con il fiore in basso (in origine in alto) nel lato nord è co-

piano, lato sud, reimpiegato in un'altra finestra (anch'esso tagliato verticalmente sui due lati al momento del reimpiego): Mazzanti op. cit. 24 fig. a p. 55 in basso al centro (è erroneo il disegno d'una croce, in luogo d'un elemento vegetale, che accompagna il volatile, così che Mazzanti ne ha fatto un inusitato volatile crucifero); Broccoli (nota 69) 223-224. 264 n. 167 tavv. 48 e 49 (dove l’immagine di Mazzanti è pubblicata capovolta); Broccoli (nota 194) 300 fig. 7 (lo dice erroneamente reimpiegato come il primo nella stessa finestra: cf. A. Guiglia Guidobaldi in: Ecclesiae Urbis [nota 6] 1503 nota 51); A. Guiglia Guidobaldi in: Ecclesiae Urbis (nota 6) 1504-1505. 4) chiesa, ambone di sinistra, reimpiegato nel parapetto della scala: cm. $102 \mathrm{~h} \times 21,5 \times 18$ : Mazzanti op. cit. 24 fig. a p. 55 in basso a sinistra; Broccoli (nota 69) 224-225. 264 nn. 168. 284 tavv. 49.69 (presenta come inedito un pezzo già pubblicato da Mazzanti, e pubblica il disegno di Mazzanti senz'accorgersi ch'è relativo al pezzo di cui sta parlando, considerandolo relativo a un pezzo oggi perduto: cf. A. Guiglia Guidobaldi in: Ecclesiae Urbis [nota 6] 1503 nota 51); A. Guiglia Guidobaldi in: Ecclesiae Urbis (nota 6) 1504-1505 fig. 11.

205 A. Guiglia Guidobaldi in: Ecclesiae Urbis (nota 6) 1504-1505 fig. 12.

${ }^{206}$ I riquadri interni alla fascia di bordo sono alti cm. 19,5 circa, ad eccezione di quello oggi in alto, che è di cm. $16 \mathrm{~h}$.

${ }^{207}$ In base all'incasso primitivo le lastre della recinzione potevano esser alte al massimo cm. 85; non c'era infatti incasso in origine in corrispondenza dell'articolato sistema di riquadrature sul lato nord in alto (oggi in basso). I lati sud e ovest del pilastrino son stati tagliati e rilavorati in funzione del reimpiego. 


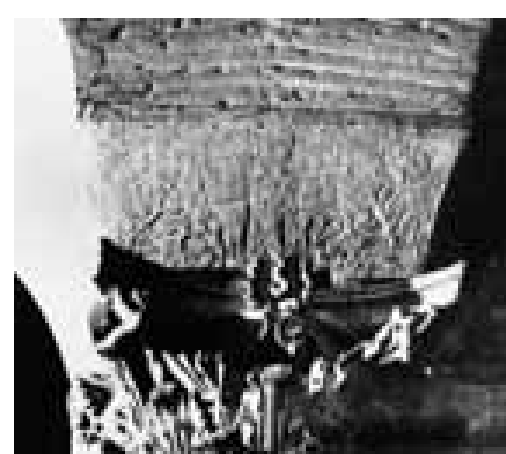

47 Roma, S. Lorenzo f. l. m.: pulvino di una delle colonne del matroneo

munque dovuto ad artefici romani. Una commistione evidente anche nel frammento di pilastrino riadoperato nella scala dell'ambone destro (fig. 44), dove alle riquadrature ${ }^{208}$ di (ipotetica) mano greco-costantinopolitana fà séguito un intervento sicuramente romano, sia pur d'imitazione, dove il disco incavato con la croce greca - la cui terminazione è tagliata a coda di rondine (identica alla croce d'uno dei pilastrini oggi nel chiostro [fig. $45]$ ) - ha il fondo non levigato e la croce irregolare e sgraziata ${ }^{209}$. Se è presente una mano greco-costantinopolitana, non è comunque di alto livello, ed è ricollegabile in qualche modo con i pilastrini del ponte Salario, mentre una connessione tra dadi e pilastrini per l'esecuzione di artefici romani d'imitazione è data dalla considerazione del volatile nel pilastrino oggi nel chiostro (fig. 45) in rapporto ai volatili sui due dadi.

I dodici pulvini sui capitelli classici di reimpiego nei colonnati del matroneo (fig. 47) ${ }^{210}$ son chiaramente coevi alla fabbrica pelagiana, di differenti altezze in funzione delle colonne classiche riadoperate, e le loro decorazioni, nello stretto collegamento con quelle dei sottostanti plinti, si rivelano prodotti locali non d'imitazione delle opere greco-costantinopolitane, al pari dei plinti, per di più resi soltanto a incisione, senza nemmeno abbassar il fondo, come invece è stato fatto nei plinti. A incisione è realizzata pure una lastra, reimpiegata come parapetto interno della scala nell'ambone destro ${ }^{211}$, che non ha ancora ricevuto la debita attenzione, segata diagonalmente in due parti, con la sinistra ricollocata capovolta sopra la destra per adeguarla all'andamento della scala. Nel XIX secolo fu vista da Ch. Rohault de Fleury, che ne parlò come se si trattasse di pezzi di lastre diverse e ne diede un disegno con i pezzi solo accostati in altra parte della sua opera, senza riferimento nel testo ${ }^{212}$; fu ripresa nel 1980 da A. Ferrua nel contesto del corpus delle epigrafi cristiane, con un altro disegno che ricongiunge i due pezzi e con esatta valutazione d'una coppia di cervi ai lati d'un cantaro ${ }^{213}$. Nel 1981 ne ha parlato U. Broccoli, che l'ha ritenuta dell'epoca di papa Pelagio, però non ne ha capito l'unitarietà, dato che secondo lui »le scene, infatti, sono contrapposte, per cui, leggendone una, l'altra risulta capovolta « ${ }^{214}$. Ricomposta la lastra mediante fotomontaggio (fig. 48), lo schema è chiaro, con una coppia di cervi, il maschio a sinistra, la femmina a destra, ai lati d'un cantaro. Motivo simbolico ricorrente su plutei e sarcofagi, realizzato con tratto non sicuro, mostra il cantaro dall'improbabile equilibrio e i cervi dall'andatura goffa, specialmente nel maschio a sinistra. È opera d'uno scalpello locale, d'imitazione dei greco-costantinopolitani, resa a incisione ${ }^{215}$ senza precisione nel solco, di livello modesto. Ma anche per le sue dimensioni ${ }^{216}$ non va considerata una parte della recinzione, e a mio parere la lastra era importante nell'arredo liturgico della chiesa, poiché credo di riconoscervi il paliotto dell'altare della basilica pelagiana.

Dal reimpiego di sculture classiche alla presenza di pezzi forse importati già rifiniti all'esecuzione in loco di pezzi da parte di maestranze fors'anche greco-costantinopolitane, oltre che romane nel duplice aspetto d'imitatori o di non imitatori dei prodotti greco-costantinopolitani, e con differenti tecniche d'esecuzione,

\footnotetext{
${ }^{208}$ La fascia è larga in alto cm. 3,5, ai lati 3 circa; il listello bombato è largo cm. 1,5; il motivo centrale a specchiatura con lato concavo aggetta sul fondo ribassato cm. 0,5.

209 Il disco ha un diametro di cm. 9 e il fondo è appena ribassato.

${ }^{210}$ Broccoli (nota 69) 226-227 n. 171 tav. 60.

${ }^{211}$ Marmo; 1) cm. $67 \mathrm{~h} \times 145$ (lungh. minima 87) $\times 4$ sp.; 2) cm. $61 \mathrm{~h} \times 75$ (lungh. minima 13) $\times 4 \mathrm{sp}$.

${ }^{212}$ Ch. Rohault de Fleury, La Messe. Études archéologiques sur ses monuments III (Paris 1883) 52 (»dessins d’agneaux et de cerfs devant un calice«); IV (1884) tav. 274 in alto (VI sec.).

${ }^{213}$ A. Ferrua, Inscriptiones christianae Urbis Romae septimo saeculo antiquiores VII (1980) 405 n. 1919a-b (Ferrua non sa se la lastra sia sepolcrale; ne dà le misure ricomposte cm. 98 × 196).

${ }^{214}$ Broccoli (nota 69) 211-213 n. 156 tav. 44: con misure sbagliate; gli animali son detti un cervo e un »quadrupede, forse un cervo (anche se qui mancano le corna, presenti nell’altro animale)«; non è ben compreso il testo di Ferrua, dato che gli si attribuisce di non aver ritrovato la lastra.

${ }^{215}$ Per il fenomeno dell'incisione vd. Russo (nota 135) 49-118 = 73-142 tavv. 13-40.

${ }^{216}$ La metà della lunghezza della lastra misura cm. 100 circa: dunque la lunghezza complessiva era di cm. 200 circa.
} 


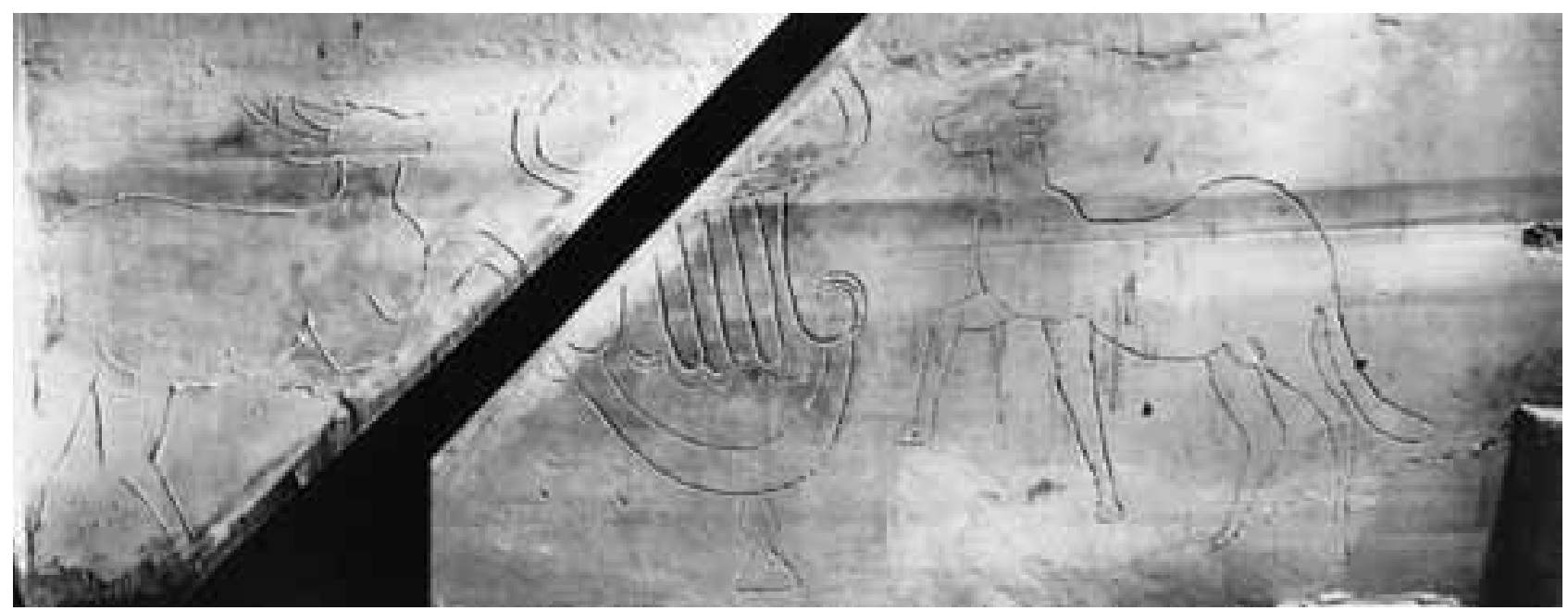

48 Roma, S. Lorenzo f. l. m.: lastra paliotto dell'altare della chiesa del VI sec., segata e reimpiegata nel parapetto interno della scala dell'ambone destro, ricomposta mediante fotomontaggio

scaturisce per S. Lorenzo un risultato di notevole eclettismo. Ma per tutto quel che s'è detto non posso condivider il giudizio limitativo della Guiglia: »Non era dunque più il tempo di rivolgersi verso la lontana Costantinopoli, ove, comunque, dopo la morte di Giustiniano, l'attività produttiva era andata sempre più rarefacendosi « ${ }^{217}$ : c'è piuttosto una stasi inventiva nella capitale, non l'incapacità di realizzare opere di alto livello, come fà fede il frammento di pluteo con rombo e croce e come vedremo meglio più avanti; a parte tutti gli stimoli e i suggerimenti che dal mondo greco-costantinopolitano son derivati alla decorazione di S. Lorenzo.

Va segnalata pure l'impronta nella malta, lasciata al momento del reimpiego come rivestimento da una lastra a S. Marcello al Corso (fig. 49) ${ }^{218}$, collegabile abbastanza strettamente con le sculture di S. Lorenzo f. l. m. e attribuibile a maestranze romane d'imitazione, di qualità non infima: la forma squadrata e vigorosa della croce, la presenza di almeno tre piccole rosette e d'un fiore a 6 petali (che in realtà sono sei losanghe), il bordo a semplice fascia, la terminazione espansa dei bracci a mo' di coda di rondine, son tutti elementi che rimandano alle sculture di S. Lorenzo f. l. m. (per le croci e le rosette, i dadi; per le terminazioni dei bracci, due pilastrini; per il fiore a 6 petali, un pilastrino), e dunque ripropongono una datazione, per questo pezzo appartenente in origine alla recinzione di S. Marcello al Corso, agli ultimi decennii del VI secolo.

Giungiamo così al presbiterio di S. Pietro in Vaticano e al tempo di Gregorio Magno. Della recinzione della fine del VI secolo identificai a suo tempo quattro frammenti: con croce entro disco ${ }^{219}$, con due pavoni ai lati d'un cantaro (fig. 50) ${ }^{220}$, con un rombo gigliato ${ }^{221}$, con il motivo a pelte ${ }^{222}$. Quest'ultimo frutto di maestranze locali, con la sua decorazione a pelte ch'abbiam visto anche in una lastra del ponte Salario: una compresenza di mani greco-costantinopolitane e di mani locali simile a quella verificata per il ponte Salario. Quest'identificazione è stata accolta dalla Barsanti unicamente come riferimento allo stesso gruppo, e soltanto per i primi tre frammenti, ma è stata con vigore indicata una datazione dei pezzi a ridosso delle lastre di S. Clemente, anche sulla scorta del fatto che il frammento con croce entro disco, una volta staccato dal muro, ha rivelato nell'altra faccia, ch'era la principale, una decorazione a schema ternario analoga a quella visibile

\footnotetext{
217 A. Guiglia Guidobaldi in: Christiana loca (nota 16) 269.

218 S. Episcopo, Il titulus Marcelli sulla via Lata. Nuovi studi e ricerche archeologiche (1990-2000) (2003) 113 (con misure $110 \times$ 80; è detta »avvicinabile per sintassi decorativa« ai due dadi di S. Lorenzo f. l. m.). Ringrazio cordialmente Silvana Episcopo per avermi fornito l'inedita fotografia e per il permesso di pubblicarla.

219 Russo (nota 1) 32 fig. 17.

${ }^{220}$ Russo (nota 1) 32-35 fig. 18.

${ }^{221}$ Russo (nota 6:1995) 10-12 fig. 3.

${ }^{222}$ Russo (nota 1) 36 fig. 19.
} 


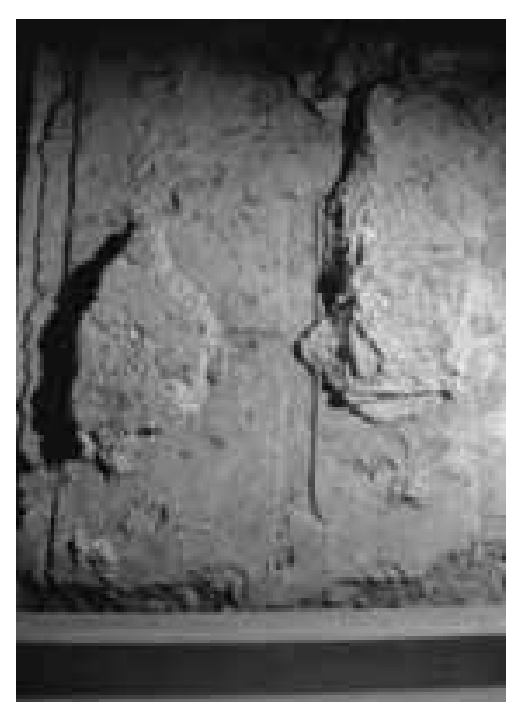

49 Roma, S. Marcello al Corso: impronta di lastra del VI sec. sulla malta d'un plinto del transetto medievale in S. Clemente ${ }^{223}$. Opinione condivisa da A. Melucco Vaccaro (secondo quarto del VI sec.) ${ }^{224}$, dalla Guiglia ${ }^{225} \mathrm{e}$ da $\mathrm{L}$. Paroli, la quale ritiene però che la lastra con i pavoni è »l'unica tra i numerosissimi plutei« delle Grotte Vaticane »che possa esser attribuita in modo convincente all'età di Gregorio Magno «226; ribadisce invece la precedente opinione la Guiglia, e ora »Resta assai dubbia la pertinenza a questo insieme omogeneo « [di due lastre] del pezzo con i pavoni ${ }^{227}$.

Mentre riconfermo ciò che scrissi nel 1979 e 1980 e ho ripetuto nel $2000^{228}$, desidero aggiunger súbito alla serie un quinto frammento, già nel giardino vaticano, oggi nel Museo Pio Cristiano, inv. 31634 (fig. 51) ${ }^{229}$, ch'è stato finora attribuito all'VIII o IX secolo ${ }^{230}$. Esso mostra uno schema decorativo analogo al frammento precedente col rombo, e coi gigli anche all'interno dei rombi: uno schema, quello coi due rombi gigliati occupanti tutto il campo e tangenti tra loro mediante i gigli, che compare già a S. Clemente ${ }^{231}$, ma in cui sia i gigli all'interno dei rombi sia soprattutto la croce greca con nastro a 2 bande all'interno dei bracci e il grande giglio bordato in basso (ch'è parte d'un più ampio motivo perduto) sono spia d'un'esecuzione decisamente posteriore ai plutei di S. Clemente. Ma le caratteristiche del pezzo pongono la sua datazione non nell'VIII secolo, bensì ancor in epoca paleocristiana, al tempo di Gregorio Magno. Da notare pure, per l'esecuzione a Roma da parte d'una maestranza grecocostantinopolitana del più alto livello (come si disse già a suo tempo per i tre primi frammenti), l'impiego di marmo non proconnesio.

E giungiamo al retro di una delle due lastre Borghese, d'età adrianea, oggi al Museo del Louvre ${ }^{232}$, quella con donne ghirlandofore e tempio (fig. 52): di recente pubblicato, conferma definitivamente la validità di

${ }^{223}$ C. Barsanti in: Guidobaldi - Barsanti - Guiglia Guidobaldi (nota 3) 130-132. 178-179 figg. 211. 273 (per il pluteo con schema ternario su una faccia, fig. 211, pubblicata capovolta, e disco con croce nell'altra, fig. 273); 132 nota 71 (per il pluteo col pavone, inserito nel gruppo "pur se con qualche perplessità«); 160. 178 fig. 244 (per il pluteo col rombo); 132 nota 71 (per il pluteo con le pelte).

${ }^{224}$ Melucco Vaccaro (nota 3:1999) 98 (evidentemente a digiuno dell'argomento di cui sta parlando, dichiara di »provenienza anatolica« il marmo proconnesio, che viene invece dalla Propontide o Mar di Marmara; a p. 99 la capsella eburnea di Samagher, che mostra il presbiterio della basilica costantiniana, è detta a »Pola, Arheološki muz. Istre«, mentr’è conservata nel Museo archeologico di Venezia).

225 A. Guiglia Guidobaldi in: Christiana loca (nota 16) 270.

${ }^{226}$ Paroli (nota 14) 133.

${ }^{227}$ A. Guiglia Guidobaldi in: Ecclesiae Urbis (nota 6) 1489. 1513.

${ }^{228}$ E. Russo in: Aurea Roma (nota 6) 196 fig. 6.

${ }^{229}$ Marmo bianco venato; cm. $64 \mathrm{~h} \times 71 \times 7$ almeno sp. superstite. Croce greca cm. $32 \times 32 \times 5-5$, 5 sp. braccia. Campo interno $\mathrm{cm}$. $67 \mathrm{~h}$ complessiva (essendo metà del campo cm. 33,5 h). L'incorniciatura è superstite in alto per $\mathrm{cm}$. $13 \mathrm{~h}$. Il rombo completo, senza gigli, doveva misurare cm. $44 \times 48$ (essendo la metà $22 \times 24$ ). Sulla superficie tracce nette di scalpello a pettine. Il frammento è in tre parti.

${ }^{230}$ Marucchi (nota 75) 13 tav. 12, 7 (»del secolo VIII o IX. Proviene dal giardino vaticano e forse appartenne all’antica basilica di S. Pietro. [Fu qui trasportato recentemente]«); C. Barsanti in: Guidobaldi - Barsanti - Guiglia Guidobaldi (nota 3) 161 nota 198 ("già attribuito all’VIII o IX secolo, meriterebbe indubbiamente uno studio specifico in rapporto alla produzione scultorea altomedievale«); A. Guiglia Guidobaldi in: Ecclesiae Urbis (nota 6) 1519-1521 fig. 24 (foto pubblicata capovolta) (»la resa stilistica è assai piatta e bidimensionale«).

${ }^{231}$ Russo (nota 1) 12 fig. 4 (con la bibl. precedente); C. Barsanti in: Guidobaldi - Barsanti - Guiglia Guidobaldi (nota 3) 85-86. 87. 89-92. 93-95. 155-157 figg. 83. 90. 96. 98. 101. 103. 105. 107-108. 110. 112. 114. 116. 121. 123-124. 129. 131-132. 134; A. Guiglia Guidobaldi in: Christiana loca (nota 14) 202-203 figg. a p. 202; eadem in: Ecclesiae Urbis (nota 6) 1483-1485 figg. 1-2.

232 1) Ma 1612, con danzatrici; marmo pentelico, cm. 71,9 h $\times 188 \times 3$ sp.: J. Charbonneaux, La sculpture grecque et romaine au Musée du Louvre (1963) 96 n. 1612; C. C. Vermeule, The Dal Pozzo-Albani Drawings of Classical Antiquities in the Royal Library at Windsor Castle (1966) 33 fol. 20 n. 8503; F. Haskell - N. Penny, Taste and the Antique. The Lure of Classical Sculpture 1500-1900 (1981) 195-196 n. 29 fig. 101; Ph. Pray Bober - R. Rubinstein, Renaissance Artists and Antique Sculpture: a Handbook of Sources (1986) 95 fig. 59A; M. E. Micheli, Rilievi con donne offerenti, danzanti e ghirlandofore a Ravenna e a Roma, Prospettiva 51, 1987 (1989) 10 n. 3 fig. 16; D. Gallo in: Dominique-Vivant Denon. L’oeil de Napoléon (Catalogue de l’Exposition, Paris, 20 octobre 1999-17 janvier 2000) 203-204 n. 208; M. E. Micheli in: L’idea del Bello. Viaggio per Roma nel Seicento con 
quanto scrissi nel 1979. La lastra mostra proporzioni sensibilmente diverse da quelle di S. Clemente, è più bassa e più lunga, e di conseguenza il campo interno, più schiacciato e allungato, deve accoglier decorazioni a esso adeguatamente rapportate, in questo caso i due rombi gigliati e tangenti, secondo uno schema già incontrato, ma ora sviluppati più in larghezza che in altezza. L'incorniciatura a 4 elementi è una semplificazione di quelle a 5 elementi non solo di S. Clemente (figg. 16. 18. 57), ma an-

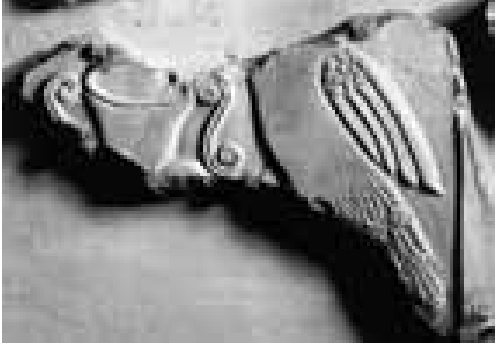

50 Roma, S. Pietro in Vaticano: frammento di pluteo della recinzione del tempo di papa Gregorio Magno (590-604), oggi nelle Grotte Vaticane

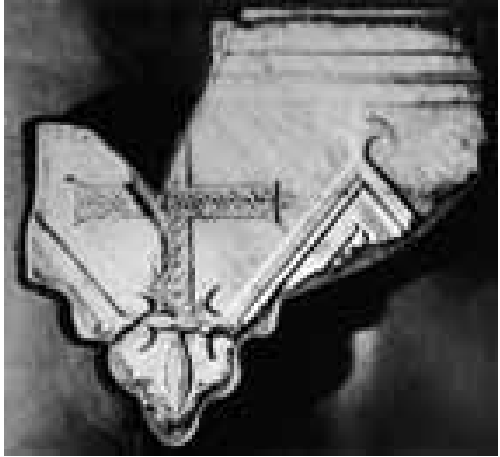

51 Roma, Museo Pio Cristiano: frammento di pluteo della recinzione di S. Pietro in Vaticano, del tempo di Gregorio Magno che delle altre lastre romane del VI secolo che le abbian conservate, come Ss. Cosma e Damiano (fig. 19), S. Maria in Cosmedin (fig. 53) ${ }^{233}$ e S. Maria in via Lata ${ }^{234}$, dato che dopo la modanatura più interna è sparita la modanatura stretta a dente; assistiamo a una semplificazione pure delle riquadrature che costituiscon i rombi, che passano dalle tre di S. Clemente (fig. 57), di S. Maria in Cosmedin (fig. 53), di S. Maria in via Lata, del Foro Romano ${ }^{235}$, di S. Lorenzo f. l. m. e dello stesso secondo frammento gregoriano di S. Pietro (oltre che del quinto [fig. 51]), a due, eliminando la modanatura stretta a dente al centro. Osservando attentamente (figg. 54. 55. 56), notiamo che il fondo, a differenza delle lastre delle Grotte Vaticane che ho attribuito al presbiterio di Gregorio III (731-741) ${ }^{236}$, è lavorato non con lo scalpello piatto, bensì con quello a pettine, mentre le decorazioni son ricavate in una prima fase con lo scalpello a pettine e in un secondo momento con lo scalpello piatto. Il motivo centrale nei rombi, vegetale, a 3 elementi su gambo tre volte ripetuti, si rifà in modo soltanto approssimativo al motivo a foglia o rametto presente in una sola lastra del complesso di S. Clemente (fig. 57) ${ }^{237}$ : esso ci permette di ricostruire con maggior esattezza il motivo che compare sulla metà superiore d'una lastra di S. Maria in Cosmedin (fig. 53) e finora non interpretato correttamente né dal disegno del Crescimbeni ${ }^{238}$ seguíto dalla Barsanti, che vi legge »un elemento pseudo-gigliato il cui stelo sembrerebbe innestarsi in una sorta di cespo del quale sono appena visibili le terminazioni superiori ${ }^{239}$, né dal disegno del Mazzanti, che vi vede quattro elementi gigliati disposti a croce $^{240}$. Si comprende bene che le forme arrotondate che si scorgon ai lati dello stelo non

Giovan Pietro Bellori (Catalogo della Mostra, Roma, 29 marzo - 26 giugno 2000) 418-419 n. 14; eadem, Rilievi con donne offerenti, danzanti e ghirlandofore a Ravenna e a Roma, II, Prospettiva 101, 2001 (2002) 46-47. 2) Ma 1641, con donne ghirlandofore e tempio; marmo pentelico; cm. 69,5 h × 186,7 × 2,8 sp.: Charbonneaux op. cit. 96 n. 1641; Pray Bober - Rubinstein op. cit. 95 n. 59B; Micheli op. cit. (1987) 11 n. 4 fig. 19; eadem op. cit. (2000) 419-420 n. 15; eadem op. cit. (2002) 47-49 figg. 5-7. All'inizio del XVII sec. i due rilievi erano nella collezione del cardinale Scipione Borghese, e furono venduti al Louvre da Camillo Borghese, cognato di Napoleone. Il retro che c'interessa è quello del rilievo Ma 1641. Ringrazio cordialmente la collega Micheli, che ha pubblicato per la prima volta, con una serie d'osservazioni pertinenti, il retro del pezzo, ibidem 47 figg. 6-7, per avermi procurato le relative fotografie del Museo del Louvre, eseguite da Chr. Larrieu.

233 Russo (nota 1) 20 (con la bibl. precedente); C. Barsanti in: Guidobaldi - Barsanti - Guiglia Guidobaldi (nota 3) 165-170. 268 figg. 249-250. 404.

${ }^{234}$ Russo (nota 1) 20-21 fig. 7 (con la bibl. precedente); C. Barsanti in: Guidobaldi - Barsanti - Guiglia Guidobaldi (nota 3) 164 fig. 248 (non si cita il mio contributo, dove ho pubblicato il pezzo).

235 1) C. Barsanti in: Guidobaldi - Barsanti - Guiglia Guidobaldi (nota 3) 158-159. 267-268 figg. 242. 401; Paroli (nota 14 ) 220 n. I.9.2 fig. a p. 221 in alto. 2) C. Barsanti in: Guidobaldi - Barsanti - Guiglia Guidobaldi (nota 3) 159-160 fig. 243; Paroli (nota 14) 220 n. I.9.1 fig. a p. 220 in alto.

${ }^{236}$ Russo (nota 6:1985) 12-33 figg. 5-17.

${ }^{237}$ C. Barsanti in: Guidobaldi - Barsanti - Guiglia Guidobaldi (nota 3) 92 fig. 114.

${ }^{238}$ G. M. Crescimbeni, L'istoria della basilica diaconale, collegiata, e parrocchiale di S. Maria in Cosmedin (Roma 1715) 134-135 fig. tra le pp. 134-135 (il terzo pluteo dall’alto). Il pezzo è pubblicato pure da G. B. Giovenale, La basilica di S. Maria in Cosmedin (1927) 331 fig. 109.

${ }^{239}$ C. Barsanti in: Guidobaldi - Barsanti - Guiglia Guidobaldi (nota 3) 168. 175-176 fig. 250.

${ }^{240}$ C. Barsanti in: Guidobaldi - Barsanti - Guiglia Guidobaldi (nota 3) 268 fig. 404. 


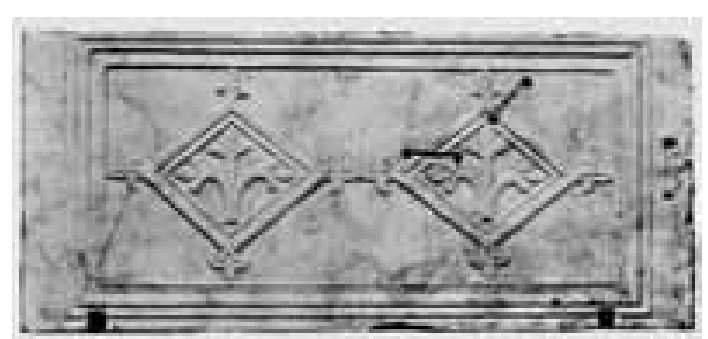

52 Parigi, Museo del Louvre: retro del rilievo Borghese con donne ghirlandofore e tempio

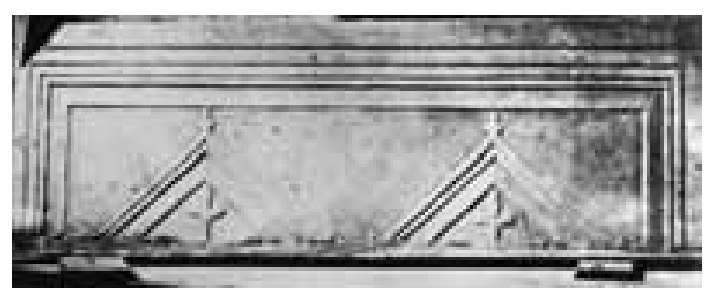

53 Roma, S. Maria in Cosmedin: frammento di lastra

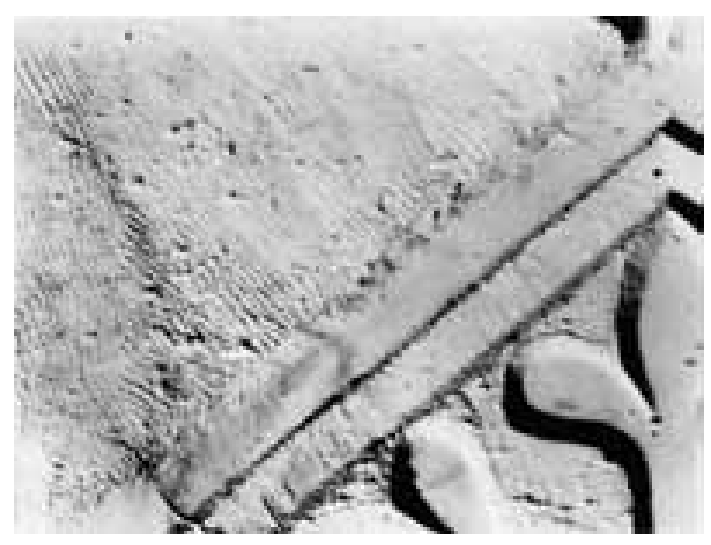

54 Parigi, Museo del Louvre: retro del rilievo Borghese con donne ghirlandofore e tempio, particolare sono né le terminazioni superiori d’un cespo né la metà rivolta verso l'alto d'una sorta di giglio, il resto d'un motivo analogo alla lastra Borghese; l'unico dubbio restante riguarda la presenza o non del cespo in basso indicato da G. M. Crescimbeni, ma sono piuttosto portato a escluderla. Appare veramente probabile che il motivo della lastra di S. Maria in Cosmedin abbia svolto la funzione di modello o fonte d'ispirazione per quello del rilievo Borghese nella certissima esecuzione a Roma di questo. E d'altra parte rispetto a S. Maria in Cosmedin è inevitabile per la lastra Borghese spostarci con la cronologia alla fine del secolo, pur sottolineando sempre l'opera d'una maestranza greco-costantinopolitana del più alto livello, data l'epoca: considerando dadi e pilastrini di S. Lorenzo f. l. m., è assolutamente impensabile l'esecuzione da parte di maestranze romane, sia pur d'imitazione.

Il motivo della lastra di S. Maria in Cosmedin è stato accostato dalla Barsanti a un "problematico « frammento - da lei pubblicato - oggi murato in un ambiente adiacente al chiostro di S. Giovanni in Laterano (fig. 58), con l'avvertenza che $»$ Le caratteristiche formali del pezzo suggeriscono estrema cautela nel definire la sua collocazione cronologica, per cui soltanto con molte riserve qui lo proponiamo come termine di confronto « ${ }^{241}$, evidentemente per indicar un'epoca posteriore rispetto a S. Maria in Cosmedin. Ebbene, il motivo vegetale nella lastra del Louvre in tutto il bacino del Mediterraneo trova nel frammento oggi a S. Giovanni in Laterano ${ }^{242}$ un confronto talmente puntuale, da risultare sovrapponibile nella forma dei motivi, nella semplificazione delle riquadrature dei rombi rispetto a S. Clemente e alle altre lastre coi rombi, nella tecnica di trattamento del fondo con lo scalpello a pettine e delle decorazioni con lo scalpello a pettine e poi con lo scalpello piatto: così che si può ritenere senz'altro il frammento finito a S. Giovanni in Laterano frutto delle stesse mani e appartenente al medesimo complesso di cui faceva parte il rilievo Borghese.

Nel 1999 è stata pubblicata una relazione del 1962 di F. Magi su uno scavo nel luogo dov'era l'antico secretarium della basilica costantiniana di S. Pietro in Vaticano, nella piazza dei Protomartiri cristiani, in stretta prossimità all'ingresso dell'arco delle Campane: il secretarium »era pieno di sepolcri, la cui serie sembra iniziarsi con quello di papa Benedetto I, morto nel 579 ... Il luogo del trovamento fu però ampiamente manomesso; ne fanno fede i pochi oggetti, frammenti di marmo e di cotto, ivi rinvenuti fra le terre, che discendono fino al Rinascimento. $\aleph^{243}$. Tra i frammenti di marmo, uno (fig. 59) ${ }^{244}$ con il rombo gigliato, pure a 2 e non a 3

${ }^{241}$ C. Barsanti in: Guidobaldi - Barsanti - Guiglia Guidobaldi (nota 3) 168-169 fig. 252.

${ }^{242}$ La Barsanti ha pubblicato il frammento con l'elemento vegetale rivolto verso l'alto: ma la sua corretta posizione si ottiene ruotando di $90^{\circ}$ l'immagine del pezzo verso sinistra.

${ }^{243}$ In P. Liverani, La topografia antica del Vaticano (1999) 147 (a p. 146 Magi ricorda che il secretarium della basilica costantiniana aveva l'aspetto »di una piccola basilica con tre cappelle lungo ciascun lato e un'abside in fondo, e comunicava attraverso l'atrio con la grande basilica della quale aveva lo stesso livello«).

${ }^{244}$ Liverani (nota 243) fig. 189. Accenna a questo frammento, per »il vertice gigliato di un rombo o di un quadrato«, A. Guiglia Guidobaldi in: Ecclesiae Urbis (nota 6) 1520 nota 71. 
elementi, pure col fondo lavorato con lo scalpello a pettine e le decorazioni ottenute con lo scalpello a pettine e poi con lo scalpello piatto, presenta all'interno un motivo vegetale talmente caratteristico e simile a quello del rilievo Borghese, da quanto si riesce a cogliere ${ }^{245}$, che non può esser disgiunto dal pezzo oggi al Louvre e dal frammento attualmente a S. Giovanni in Laterano.

Orbene, per il rilievo Borghese con donne ghirlandofore e tempio, della donna che reca frutti si conserva un disegno ${ }^{246}$ della bottega di Domenico Ghirlandaio nel Codex Escurialensis 28-II-12, dell'ultimo quarto del XV secolo ${ }^{247}$, al fol. $51^{\mathrm{v} 248}$, con nota manoscritta »in sulla piaza di sancto pietro ${ }^{249}$. A. Michaelis, ignaro della decorazione nel retro del rilievo Borghese, pensò a un palazzo sulla piazza, dove c'era un tempo la casa di Marco Cornaro Veneto o palazzo Cesi ${ }^{250}$, e quest'opinione è stata pure di recente ripetuta ${ }^{251}$. Ma oggi,

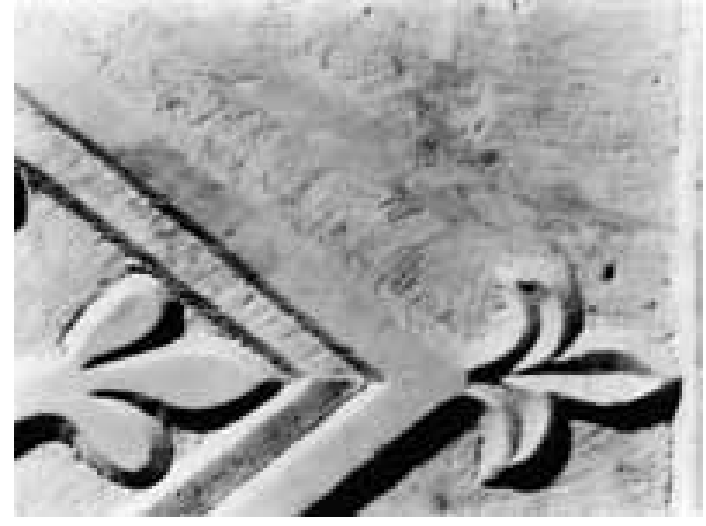

55 Parigi, Museo del Louvre: retro del rilievo Borghese con donne ghirlandofore e tempio, particolare grazie al retro del rilievo Borghese, sappiamo che l'indicazione si riferiva alla basilica vaticana. In tal modo il cerchio si è chiuso, nel riferimento del rilievo Borghese, del frammento di S. Giovanni in Laterano, del frammento dallo scavo del secretarium della basilica vaticana al medesimo complesso gregoriano di S. Pietro in Vaticano ${ }^{252}$.

I rilievi Borghese oggi al Louvre sono però due, il secondo raffigura danzatrici. Il suo retro, che pure presento (fig. 60) ${ }^{253}$, a differenza dell'altro non è tuttavia decorato. Ciò ci pone un ulteriore problema: perché non reca alcuna decorazione? Non è infatti pensabile che i due rilievi fossero disgiunti nell'ultimo quarto del '400. Son oggi uniti soltanto perché, quando cessò la loro funzione di lastre di recinzione o copertura in chiesa, furon mantenuti uniti, come uniti erano allorché furon reimpiegati alla fine del VI secolo. Il retro del secondo rilievo Borghese non reca decorazioni, ma è accuratamente levigato: dunque fu adoperato anch'esso, ma senza decorazione, in un punto in cui evidentemente la decorazione, per la sua funzione, non era necessaria. Ora, dato lo spessore esiguo delle due lastre oggi al Louvre, inadatte per questo a funger da recinzione libera, e a mo-

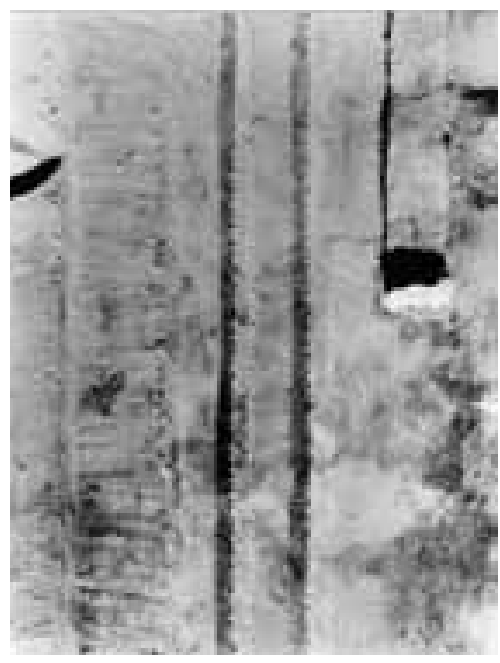

56 Parigi, Museo del Louvre: retro del rilievo Borghese con donne ghirlandofore e tempio, particolare

${ }^{245}$ L'unica differenza concreta riguarda la forma del giglio, nel frammento del secretarium più tozza e larga, e meno elegante, rispetto al rilievo Borghese. Ma è un problema di qualità di mano, non di distanza cronologica.

${ }^{246}$ Nel disegno la donna reca frutti non in mano, ma su un piatto.

${ }^{247}$ H. Egger, Codex Escurialensis. Ein Skizzenbuch aus der Werkstatt Domenico Ghirlandaios (1905/06) 44-56.

248 A. Michaelis in: Egger (nota 247) 129 fol. 51; M. Fernández Gómez, Codex Escurialensis 28-II-12. Libro de dibujos o antigüedades (2000) 109-110 fig. a p. 109 in basso.

${ }^{249}$ Questa è la grafía esatta. A. Michaelis in: Egger (nota 247) 129, legge »insulla piaza disancto pietro«; Pray Bober - Rubinstein (nota 232) 95 n. 59B, »in sulla piaza di santo piero«; la Fernández Gómez (nota 248) 109-110, come A. Michaelis.

250 A. Michaelis in: Egger (nota 247) 129.

${ }^{251}$ Fernández Gómez (nota 248) 109-110 (»en una casa de la plaza del antiguo San Pedro«).

${ }^{252}$ Anche il numero delle lastre ricuperate, nove compresi i cinque frammenti precedenti, tutte riferibili alla medesima epoca, rende inconsistente una collocazione cronologica prima dell'epoca di Gregorio Magno, e relega al rango di congettura fantastica l'ipotesi di »un rinnovamento, forse anche solo parziale, delle recinzioni nell’ambito del santuario petriano« (A. Guiglia Guidobaldi in: Ecclesiae Urbis [nota 6] 1489), giacché della recinzione costantiniana abbiamo una ricostruzione attendibile (cf. Russo [nota 6:1985] 5), a differenza di quella gregoriana (ibidem 5-6), e comunque soltanto in presenza del presbiterio rialzato è possibile il tentativo di collocamento di tutti i pezzi. Né potrebbe valere un'ipotesi che volesse impiegati contemporaneamente nella basilica martiriale di S. Pietro i plutei costantiniani e del V sec. della recinzione (su cui vd. ibidem 9 figg. 1-2) e i plutei che ho attribuito a Gregorio Magno in ulteriori altre recinzioni del VI sec., prima della creazione del presbiterio rialzato.

${ }^{253}$ Devo la fotografia del retro del rilievo Borghese Ma 1612 alla gentilezza del prof. J.-L. Martinez, conservatore capo del Département des Antiquités grecques, etrusques et romaines del Museo del Louvre, che cordialmente ringrazio. La foto è stata eseguita da P. Lebaude. 


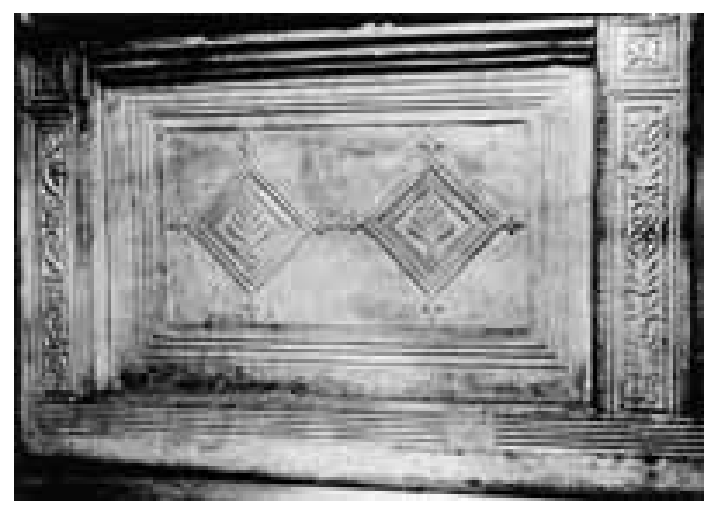

57 Roma, S. Clemente: pluteo con rombi della recinzione di papa Giovanni II

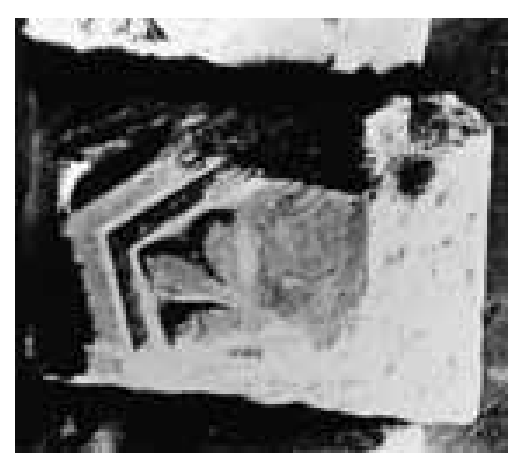

58 Roma, S. Giovanni in Laterano, ambiente adiacente al chiostro: frammento di lastra della recinzione di S. Pietro in Vaticano

tivo delle decorazioni classiche, del tutto stridenti con l'ambiente in cui avveniva il reimpiego, è più che probabile che i rilievi Borghese sian stati riadoperati nel presbiterio rialzato di Gregorio Magno come rivestimento e non nel recinto.

Abbiamo dunque ricuperato finora nove lastre dell'epoca gregoriana del presbiterio di $\mathrm{S}$.

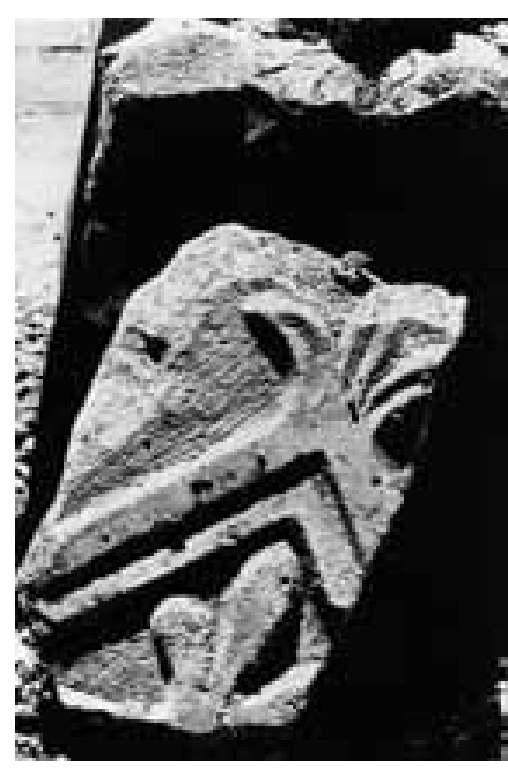

59 Roma, scavo del secretarium di S. Pietro in Vaticano: frammento di lastra della recinzione di S. Pietro in Vaticano

Pietro in Vaticano. I rilievi Borghese smentiscono la fantasiosa attribuzione all'epoca di Gregorio Magno ${ }^{254}$ di parte delle lastre ascrivibili invece all’VIII secolo, al pontificato di Gregorio III (731-741) ${ }^{255}$. Devo ribadire con forza la presenza a Roma, al massimo livello, delle maestranze greco-costantinopolitane anche alla fine del VI secolo: una presenza che - ammettendo un momento per assurdo la fantasiosa attribuzione di cui sopra - sarebbe altrettanto esplicita anche in riferimento a quel gruppo di lastre, sicuramente frutto di scalpelli greco-costantinopolitani e non locali, a smentire comunque la ricostruzione del quadro del VI secolo a Roma ch'è stato da altri proposto. Quando si cerca a Roma d'imitare troppo puntualmente la produzione greco-costantinopolitana, da parte di maestranze che non possiedono non soltanto la tecnica, ma neppure la sensibilità formale proprie di quel mondo, si ottengon esiti come quello offerto da un frammento di lastra, della fine del VI secolo, oggi nel Museo della Crypta Balbi, proveniente dalla raccolta del Foro Romano (fig. $61)^{256}$.

Mi rendo conto che appare a prima vista sconcertante - ma è l'unica possibile - la collocazione all'VIII secolo delle altre lastre di recinzione delle Grotte Vaticane, cui sono da affiancare i due pilastrini di recinzione già reimpiegati a sostenere la mensa dell'altare nella cappella delle Partorienti nelle medesime Grotte ${ }^{257}$, che feci fotografare nel 1980 (figg.

${ }^{254}$ C. Barsanti in: Guidobaldi - Barsanti - Guiglia Guidobaldi (nota 3) 160-161 (con schema a 2 rombi tangenti) fig. 245; Melucco Vaccaro (nota 3:1999) 99 (con due quadrati intrecciati; »produzioni bizantine di importazione«); A. Guiglia Guidobaldi in: Christiana loca (nota 16) 270 (con due quadrati intrecciati; »sono scolpite nel marmo lunense e ci riconducono quindi ad una creazione locale«); eadem in: Ecclesiae Urbis (nota 6) 1514-1519 figg. 20-23 (con due quadrati intrecciati e con due rombi tangenti; marmo lunense); 1521-1523 figg. 25-27 (due pilastrini: età di Gregorio Magno?). Contra vd. anche E. Russo in: Ecclesiae Urbis (nota 6) 1629-1632.

255 Russo (nota 6:1985) 12-33 figg. 5-17. Sono seguíto dalla Paroli (nota 14) 135.

${ }^{256}$ Paroli (nota 14) 134. 221 n. I.9.3 fig. a p. 222 in alto (con datazione, che non condivido, alla fine del VII sec.).

257 1) Pilastrino reimpiegato a sinistra, capovolto. Marmo grigiastro; cm. 118,5 h $\times 33,5$ largh. $\times 20$ sp.; incasso cm. 6 abbondanti $\times$ 2 prof. Nella faccia esterna da una cornucopia in basso fuoresce un tralcio vegetale con motivi vegetali a semifoglia; nel quadrato superiore due melograni. Nella faccia interna le consuete riquadrature, coi lati corti concavi, e un riquadro superiore, che però non contiene decorazioni. 2) Pilastrino reimpiegato a destra, capovolto. Marmo grigiastro con venature; cm. 118,5 h $\times 33$ circa largh. $\times 20$ sp.; incasso cm. 6 abbondanti $\times 2$ circa prof. Nella faccia esterna da una cornucopia fuoresce un tralcio: ma la decorazione, probabilmente solo abbozzata, è in parte consunta dal calpestío di un precedente reimpiego; a metà il tralcio doveva interrompersi, dato che seguono verso l'alto quattro riquadri: un motivo vegetale con tre gigli, un largo vaso con alto piede su cui è adagiato un volatile, un altro motivo vegetale pare pure con gigli, una curiosa rappresentazione di una brocca biansata dal lungo collo, rovesciata. Nella faccia interna le consuete riquadrature con i lati corti concavi, e un riquadro superiore che a differenza dell'altro contiene un motivo a giglio. 
62-64) $)^{258}$. Il complesso di Gregorio III, frutto di maestranze pure del più alto livello, in parte è agganciato sul piano decorativo ai rigidi modelli del VI secolo, cui si riporta 'archeologicamente', in parte se ne allontana per seguire una più libera inventiva ${ }^{259}$, e costituisce un insieme di straordinaria importanza nel panorama coevo dell'VIII secolo: il confronto con la contemporanea lastra eseguita dal maestro Orso e conservata nell'abbazia di S. Pietro in Valle a Ferentillo260 fà toccare con mano l'abisso che sul piano qualitativo divide tali opere e la ricchezza e la complessità delle coordinate culturali che le sculture vaticane presentano; e se greco-costantinopolitane appaion le maestranze di tali sculture, nondimeno esse son nutrite d'una più vasta cultura ${ }^{261}$, anche in considerazione di quanto va emergendo sempre più chiaramente a proposito dell'arte del periodo omayyade in Palestina e Giordania ${ }^{262}$.

Ci siamo soffermati sulla scultura architettonica, ma evidentemente il fenomeno della presenza di maestranze greco-costantinopolitane a Roma nel VI secolo è assai più vasto, e non potrò trattare analiticamente tutti gli aspetti che rivelano l'opera di tali artefici nell'Urbe.

Nell'àmbito dell'architettura, a esempio, non possiamo non ricordare la chiesa di S. Giovanni a porta Latina (fig. 65) ${ }^{263}$, costruita a mio parere sicuramente da un architetto greco-costantinopolitano dopo la conquista

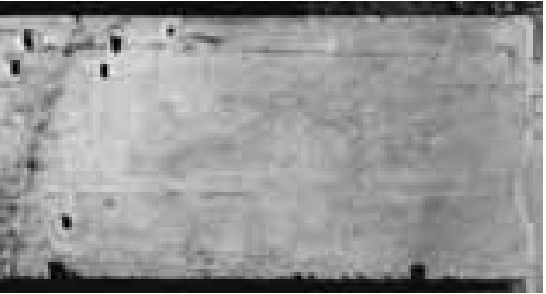

60 Parigi, Museo del Louvre: retro del rilievo Borghese con danzatrici

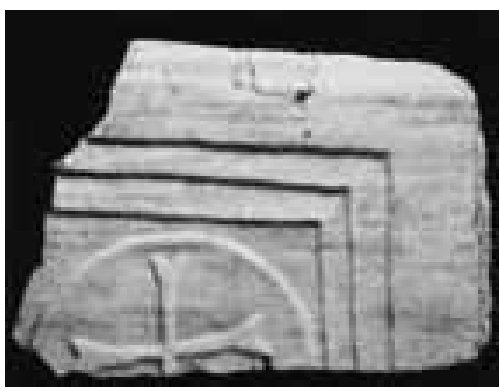

61 Roma, Museo della Crypta Balbi: frammento di lastra, dalla raccolta del Foro Romano di Roma, poco prima della conquista di Ravenna; la chiesa è culturalmente legata all'Asia Minore e a Costantinopoli per via della presenza dell'abside poligonale all'esterno ${ }^{264}$ e di due ambienti laterali all'abside e muniti di absidiole ${ }^{265}$; inoltre l'edificio ha l'abside a est. Rammento che le chiese di Roma presentano l'abside semicircolare anche all'esterno, son orientate a ovest e non mostrano ambienti laterali all'abside. È veramente impossibile parlare di ‘influenze’ orientali, come fà invece F. Guidobaldi ${ }^{266}$ : sono scelte dovute a uno o più architetti orientali presenti al momento a Roma, giacché i romani non avevan motivo di subire tali influenze nel campo dell'architettura.

${ }^{258}$ Le immagini furon realizzate a mie spese, dietro mia richiesta e su mia direttiva sul posto, dal fotografo Solazzi per conto della Reverenda Fabbrica di San Pietro, nell’àmbito della mia ricerca sulle sculture dell’antico presbiterio, ma le ho poi viste pubblicate da A. Guiglia Guidobaldi in: Ecclesiae Urbis (nota 6) 1521-1523 figg. 25-27, senza misure o osservazioni dirette, evidentemente senza aver visto i pilastrini. Per questo ho deciso di pubblicare ora i pilastrini.

259 Soprattutto i pezzi in Russo (nota 6:1985) 24-28 figg. 15-17.

${ }^{260}$ Vd. da ultimo E. Russo, Immagine e narrazione nella scultura dell’VIII secolo, in: Medioevo: immagine e racconto. Atti del Convegno internazionale di studi, Parma, 27-30 settembre 2000 (2003) 142. 157 (con la bibl. precedente).

${ }^{261}$ Cf. Russo (nota 260) 142-161.

${ }^{262}$ Per tale fenomeno vd. da ultimo O. Grabar, The Formation of Islamic Art (1973) e H. G. Franz, Palast, Moschee und Wüstenschloss I (1984) 37-103 (con bibl. precedente, II [1984] 167-181). Per la scultura rimando a Russo (nota 7) passim 113-248; e - oltre ai numerosi contributi di scavo e di esplorazione in Giordania pubblicati da M. Piccirillo nel »Liber annuus « e sintetizzati con panoramica generale in M. Piccirillo, L’Arabia cristiana. Dalla provincia imperiale al primo periodo islamico (2002), cui è da affiancare A. Michel, Les églises d'époque bizantine et umayyade de la Jordanie, Ve-VIII ${ }^{\mathrm{e}}$ siècle (2001) - ad A. Acconci in: Piccirillo - Alliata (nota 34:1994) 290-313; eadem in: Piccirillo - Alliata (nota 34:1998) 468-549; J. P. Sodini in: Les églises de Jordanie (nota 34) 123-145; e alle altre opere già ricordate nella nota 34.

${ }^{263}$ R. Krautheimer, Corpus basilicarum christianarum Romae I 4 (1953) 309-312. 314-316 tav. 40 figg. 164-165; R. e W. N. Schumacher, Die Kirche San Giovanni a Porta Latina, Kölner Domblatt 12/13, 1957, 22-38; W. N. Schumacher, Byzantinisches in Rom, RömQSchr 68, 1973, 104-124. Per le strutture murarie M. Cecchelli, Le strutture murarie di Roma tra IV e VII secolo, in: Materiali e tecniche (nota 17) 90; A. Milella, S. Giovanni a Porta Latina, in: ibidem 264-268.

264 Per l'abside poligonale all'esterno vd. Russo (nota 8:2003) 3.

265 Per gli ambienti laterali all'abside muniti di absidiole vd. Russo (nota 8:2003) 76-79.

266 Anzi, l'influenza, parlando soltanto dell'abside poligonale, è detta ravennate: »resta dubbio se la novità possa essere attribuita a influenza ravennate pregiustinianea o a una più diretta importazione culturale costantinopolitana di poco precedente o successiva alle guerre gotiche« (F. Guidobaldi, s. v. Roma, in: Enciclopedia dell’arte medievale X [1999] 68). 


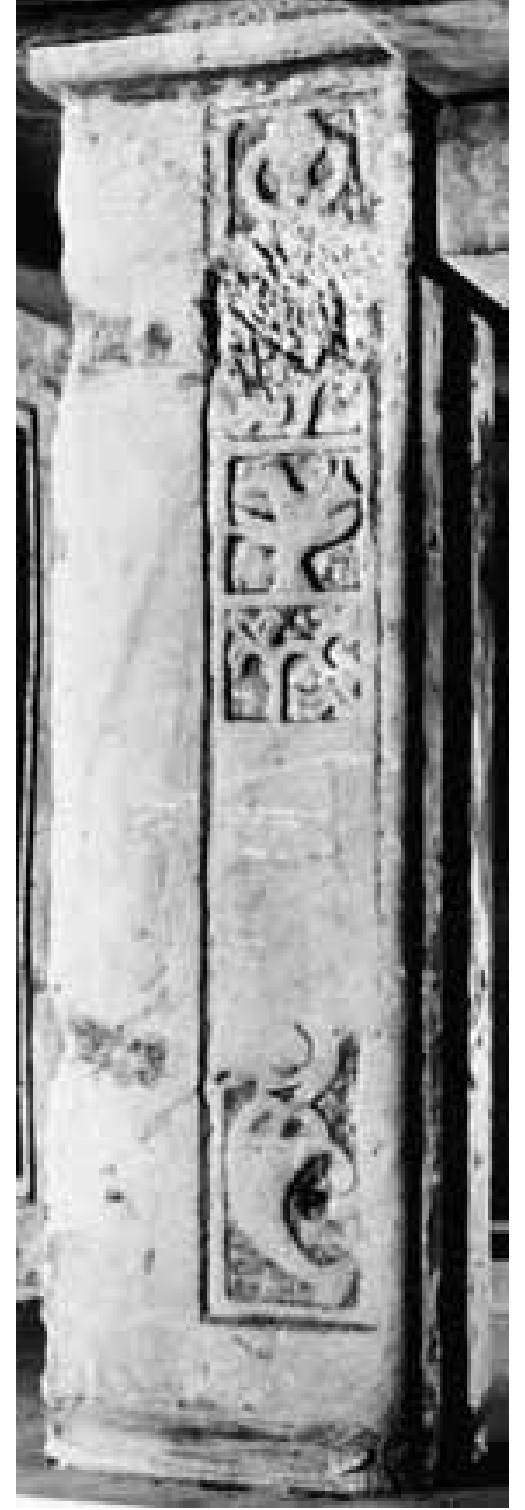

62 Roma, S. Pietro in Vaticano: pilastrino già reimpiegato a destra nell'altare della cappella delle Partorienti nelle Grotte Vaticane

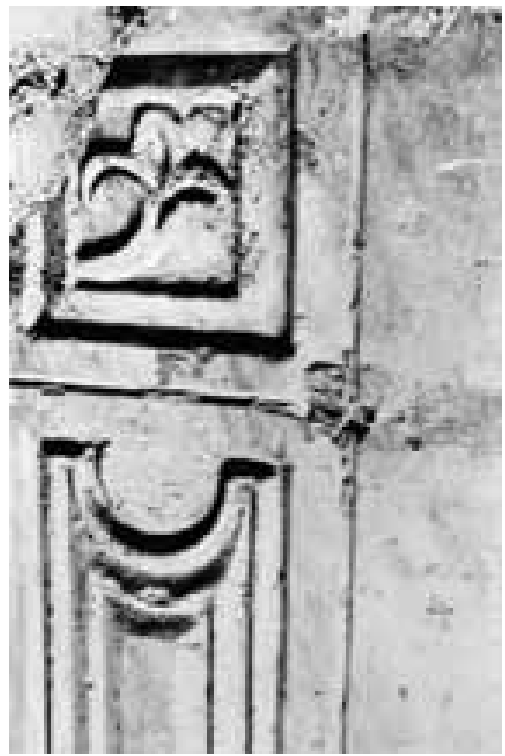

63 Roma, S. Pietro in Vaticano: pilastrino già reimpiegato a destra nell'altare della cappella delle Partorienti nelle Grotte Vaticane, particolare

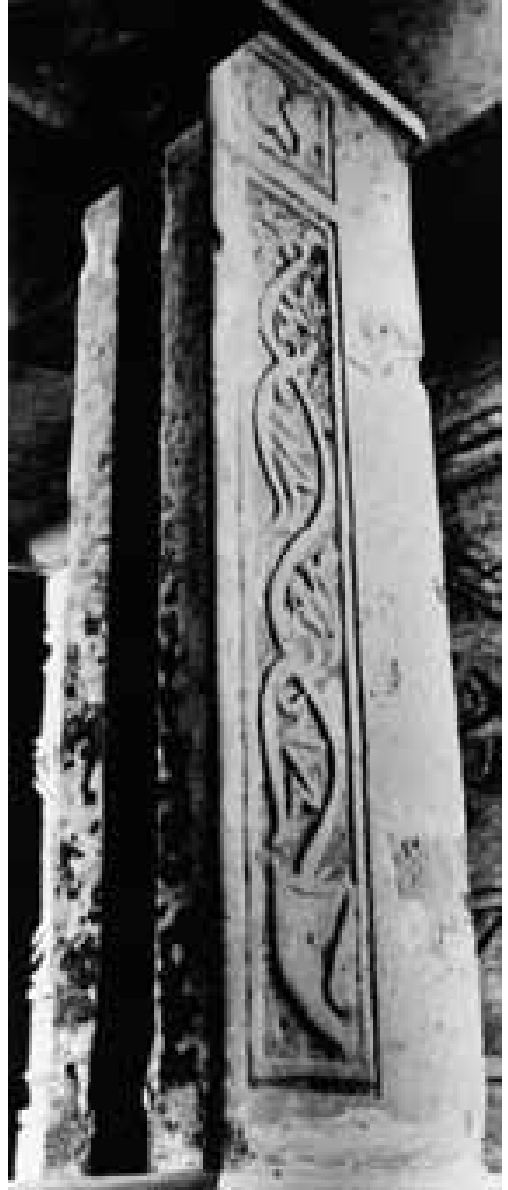

64 Roma, S. Pietro in Vaticano: pilastrino già reimpiegato $\mathrm{a}$ sinistra nell'altare della cappella delle Partorienti nelle Grotte Vaticane

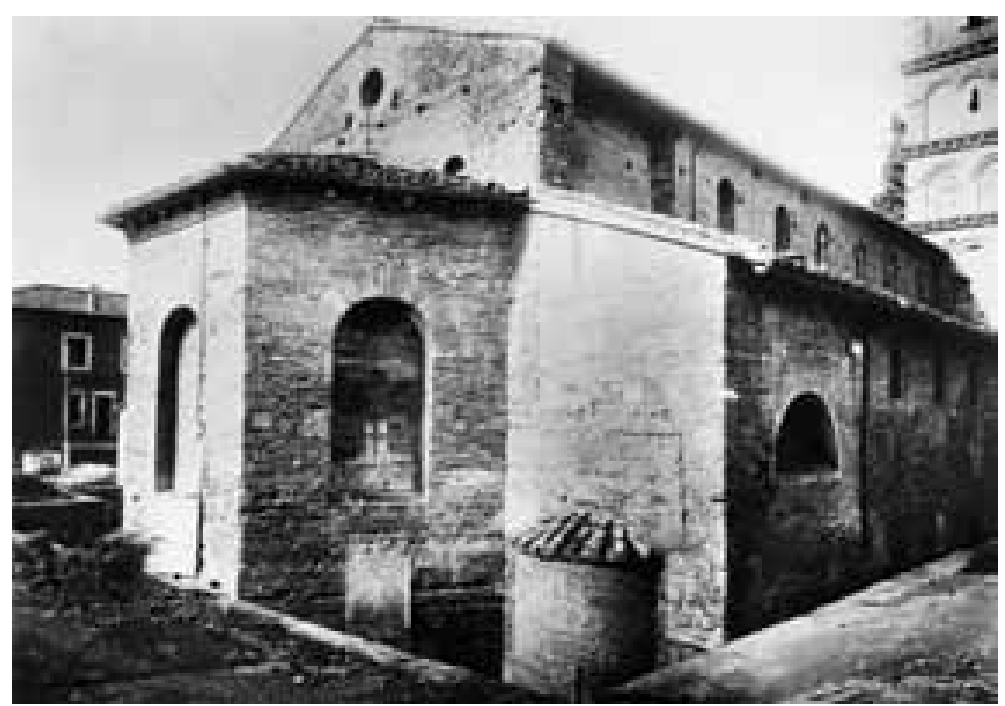

65 Roma, S. Giovanni a porta Latina: la parte absidale 
Una presenza di greco-costantinopolitani molto più diffusa di quanto si possa pensare sulle prime. Consideriamo i pavimenti, su cui possediamo le meritorie ricerche di $\mathrm{F}$. Guidobaldi e A. Guiglia ${ }^{267}$. A Roma c'era una secolare tradizione al riguardo, mentre col VI secolo il quadro muta, giacché assistiamo all'introduzione dell'opus sectile geometrico a piccoli elementi, caratteristico dell'Oriente ${ }^{268}$. Mi limito agli esempi dell'ambiente d'ingresso (fig. 66) ${ }^{269}$ e della taberna VIII (fig. 67) ${ }^{270}$ nella basilica Emilia al Foro e alla zona del presbiterio di S. Maria Antiqua (fig. 68) ${ }^{271}$. A Roma le maestranze per i pavimenti eran esperte: perché avrebbero dovuto improvvisamente cambiare la tecnica e le modalità di esecuzione ${ }^{272}$ ? La spiegazione corretta del fenomeno viene invece dalla considerazione che son all'opera maestranze greco-costantinopolitane ${ }^{273}$, le quali importano e applicano a Roma le proprie modalità di realizzazione dei pavimenti, naturalmente con i materiali che reperiscono sul posto, e permeabili e attente alla realtà culturale in cui si trovano ad agire. E le peculiarità dell’Urbe rispetto all'Oriente, sia per l'uso di porfidi sia per l'adozione di composizioni a 'isodomo listellato', vanno spiegate con l'applicazione del criterio dell'interazione uomo-ambiente che ho esposto al principio: le maestranze greco-costantinopolitane non son rimaste insensibili alla realtà culturale di Roma, e negli invero non numerosi pavimenti da loro realizzati direttamente - i quali si collocano in un arco cronologico abbastanza ristretto in relazione alla fortuna di altre tipologie - han applicato alcu-

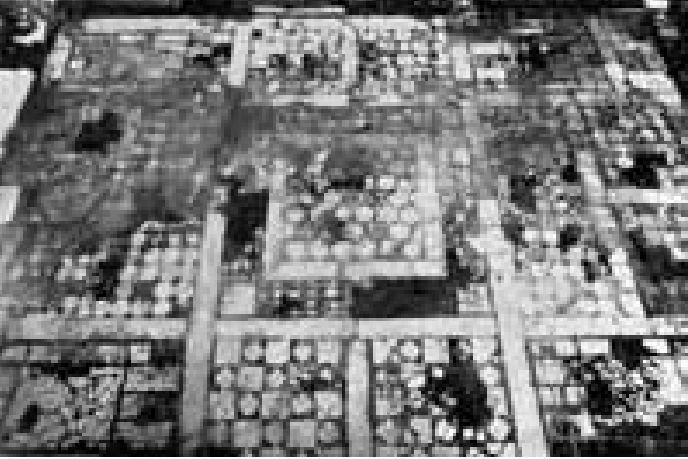

66 Roma, Foro Romano, basilica Emilia: ambiente d'ingresso, pavimento

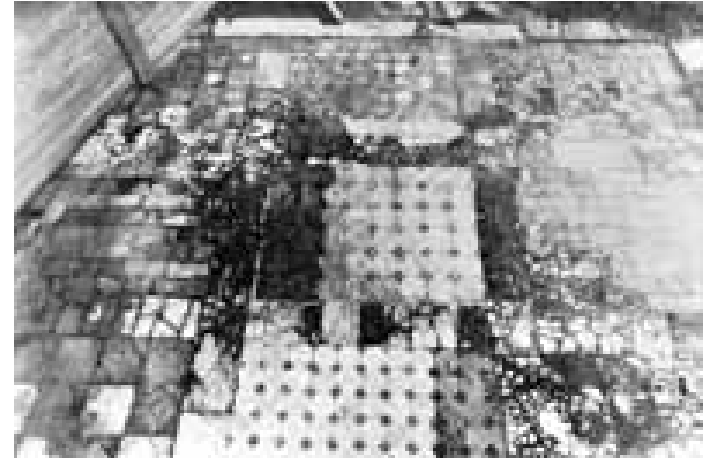

67 Roma, Foro Romano, basilica Emilia: taverna VIII, pavimento ne delle consuetudini e delle prassi esecutive proprie dell'ambiente romano. Va quindi a mio parere esattamente rovesciata la conclusione cui è giunta A. Guiglia: per i pavimenti a opus sectile geometrico a piccoli elementi, gli operatori sono da considerare in massima parte greco-costantinopolitani.

D’altro canto soltanto con una presenza bizantina a Roma si può spiegare la diffusione del culto della Vergine nell’Urbe, dopo la tappa fondamentale costituita dalla »memoria di S. Maria« almeno dall’età di

${ }^{267}$ F. Guidobaldi - A. Guiglia Guidobaldi, Pavimenti marmorei di Roma dal IV al IX secolo (1983).

268 A. Guiglia Guidobaldi, L'opus sectile pavimentale in area bizantina, in: Atti del I Colloquio dell’AISCOM (Ravenna, 29 aprile - 3 maggio 1993) (1994) 644-656.

269 A. Guiglia Guidobaldi in: Guidobaldi - Guiglia Guidobaldi (nota 267) 264-275 figg. 79-80 tav. 1, 6; eadem, I pavimenti in opus sectile delle tabernae della Basilica Emilia: testimonianze bizantine a Roma nel VI secolo, in: R. Farioli Campanati (ed.), III Colloquio internazionale sul mosaico antico (Ravenna, 6-10 settembre 1980) (1984) 505 figg. 1-3.

270 A. Guiglia Guidobaldi in: Guidobaldi - Guiglia Guidobaldi (nota 267) 276-277 fig. 84; eadem in: Farioli Campanati (nota 269) 505-507 fig. 4.

${ }^{271}$ A. Guiglia Guidobaldi in: Guidobaldi - Guiglia Guidobaldi (nota 267) 280-294 figg. 86-92 tav. 2, 1; eadem, Pavimenti marmorei a Roma e nel suburbio nei secoli IV-VII, in: Materiali e tecniche (nota 17) 200-201 fig. 9.

272 È questa invece la conclusione cui giunge la Guiglia, che ritiene le maestranze locali sia per i »pannelli contenenti composizioni collegabili con la tipologia ad sisodomo listellato<, motivo sostanzialmente estraneo al repertorio orientale ma diffuso invece a Roma stessa in tutti i secoli dell’Impero«, sia per l' »insistenza con la quale vengono usate le listellature, sempre porfiretiche, per bordare fasce divisorie e interi pannelli« (A. Guiglia Guidobaldi in: Guidobaldi - Guiglia Guidobaldi [nota 267] 341-348. 505-508; eadem in: Farioli Campanati [nota 269] 510-511). Conclusione accolta da Pensabene (nota 13) 294, e ribadita anche recentemente da A. Guiglia Guidobaldi in: Materali e tecniche (nota 17) 202 (»le componenti stilistiche, i materiali e probabilmente anche gli operatori sono da considerare in gran parte locali. Ciò è dimostrato soprattutto dalla presenza dei porfidi, il cui uso intensivo basta da solo a testimoniare la continuità di una tradizione specificamente romana«).

273 In tal senso mi ero espresso già nel 1980 in sede di discussione dopo la comunicazione della Guiglia al Colloquio sul mosaico antico (E. Russo in: Farioli Campanati [nota 269] 513). 


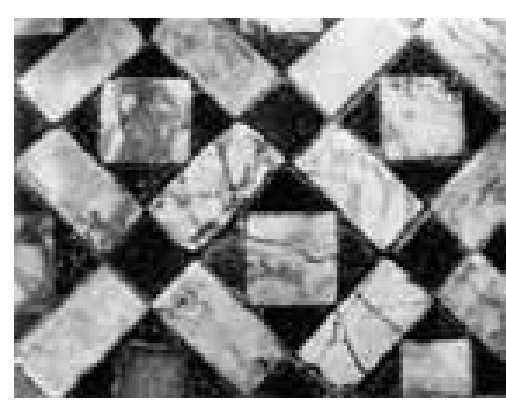

68 Roma, S. Maria Antiqua: pavimento nella zona del presbiterio

Sisto III, in collegamento con la costruzione di S. Maria Maggiore ${ }^{274}$. Diffusione del culto della Madonna che avviene in tutta Italia a séguito della conquista, mediante la costruzione e la dedica di chiese ${ }^{275}$, soprattutto per impulso di Costantinopoli. È il caso di S. Maria Maggiore di Ravenna, ricordata dallo storico Agnello come fondazione del vescovo Ecclesio, e collocabile dopo la Pasqua del 526 e non oltre il 532, anno di morte del presule ${ }^{276}$ : ed è significativo che l'edificazione avvenne per un preciso motivo, poiché Ecclesio intraprese l'opera al ritorno dalla missione a Costantinopoli, dove aveva accompagnato papa Giovanni $\mathrm{I}^{277}$. In egual modo dopo la vittoria bizantina fu eretta, come credo d'aver altrove dimostrato, S. Maria Maggiore di Bologna278; in egual modo dopo la riconquista bizantina d'Italia e prima dell'invasione longobarda si pone a mio parere la realizzazione di S. Maria di Castelseprio ${ }^{279}$, la cui datazione tarda non trova motivazioni valide né in campo archeologico né in quello formale per quanto attiene agli affreschi ${ }^{280}$. In tale contesto si colloca a Roma la cristianizzazione dell'edificio ai piedi della parte nord-ovest del Palatino, prima della metà del VI secolo, e la sua trasformazione in chiesa, con l'apertura dell'abside e la sostituzione di colonne a pilastri, all'epoca di Giustino II (565-578), con il nome di S. Maria Antiqua ${ }^{281}$. È già stata osservata la similitudine in pianta ${ }^{282}$ con la chiesa di Qașr ibnWardān ${ }^{283}$, che con il palazzo e la caserma si datava alla fine del regno di Giustiniano ${ }^{284}$, ma la conclusione

${ }^{274}$ Su tutto il problema rimando a E. Russo, L'affresco di Turtura nel cimitero di Commodilla, l'icona di S. Maria in Trastevere e le più antiche feste della Madonna a Roma [seconda parte], Bullettino dell'Istituto storico italiano per il medio evo e Archivio Muratoriano 89, 1980/81 (1982) 71-143. Per un quadro complessivo della liturgia a Roma vd. A. Chavasse, La liturgie de la ville de Rome du V ${ }^{e}$ au VIII ${ }^{e}$ siècle. Une liturgie conditionnée par l'organisation de la vie in Urbe et extra muros (1993).

275 Per il fenomeno in generale vd. P. Verzone, Da Bisanzio a Carlomagno (1968) 78.

276 Agnellus, Liber Pontificalis Ecclesiae Ravennatis, in: O. Holder Egger (ed.), Monumenta Germaniae Historica, Scriptores rerum Langobardicarum et Italicarum (Hannover 1878) 318; A. Testi Rasponi (ed.), Rerum Italicarum Scriptores² II 3 (1924) 163-166 (cap. 57). Per la più recente bibl. e le opinioni sulla chiesa vd. E. Russo, Ricerche sulla Bologna paleocristiana e medievale: la chiesa di S. Maria Maggiore, Bullettino dell'Istituto storico italiano per il medio evo e Archivio Muratoriano 84, 1972/73 (1977) 121; G. De Angelis d’Ossat, La basilica di S. Maria Maggiore a Ravenna e le dimore sovrane nella II Regio, in: XXII Corso di cultura sull'arte ravennate e bizantina (1975) 145-156; Deichmann (nota 2:1976) 343-348; Deichmann (nota 2:1989) 177.316. 371; Russo (nota 274) 141; G. Cortesi, Due basiliche ravennati del VI secolo, in: XXX Corso di cultura sull'arte ravennate e bizantina (1983) 49-67; E. Russo, L’antico portale di S. Maria Maggiore di Ravenna, RACr 60, 1984, 343-350.

277 Cf. Russo (nota 276:1977) 121.

278 Su questa chiesa vd. Russo (nota 276:1977) 21-123: come estremo cronologico »non appare agevole spostarsi troppo dalla data stessa dell'invasione longobarda« (121).

279 Rimando, anche per la bibl. precedente, a E. Russo, Il contributo di Mario Salmi agli studi sull'arte dell'età paleocristiana e altomedievale, in: Studi di storia dell'arte sul Medioevo e il Rinascimento nel centenario della nascita di Mario Salmi. Atti del Convegno internazionale, Arezzo - Firenze, 16-19 novembre 1989 (1993) 38-42 figg. 1-2. Successivamente vd. S. Lusuardi Siena - M. Andaloro, s. v. Castelseprio, in: Enciclopedia dell'arte medievale IV (1993) 447-459; G. De Spirito, À propos des peintures murales de l'église Santa Maria foris portas de Castelseprio, CArch 46, 1998 (1999) 23-64 (che, 53, data la chiesa tra il 574 e il 591).

${ }^{280}$ Vorrei segnalare che anche C. Brandi, Castelseprio, in: Terre d'Italia (1991) 118-120 (testo del 1979 pubblicato postumo), ha attribuito gli affreschi a epoca antica, il VII sec., per il confronto (peraltro già proposto da A. De Capitani d'Arzago nel 1948) con il c.d. Angelo bello di S. Maria Antiqua a Roma.

${ }^{281}$ R. Krautheimer, Corpus basilicarum christianarum Romae II (1962) 251-270.

${ }^{282}$ Da B. Brenk, Kultgeschichte versus Stilgeschichte: von der 'raison d'être’ des Bildes im 7. Jahrhundert in Rom, in: Uomo e spazio nell'alto medioevo. L Settimana di studio del Centro italiano di studi sull'alto medioevo, 4-8 aprile 2002 (2003) 1005 fig. 20.

${ }^{283}$ H. C. Butler, Syria. Publications of the Princeton University Archaeological Expeditions to Syria in 1904/05 and 1909. Division II: Architecture. Section B: Northern Syria 1 (1907) 26-45; J. Lassus, Sanctuaires chrétiens de Syrie (1947) 146-147; C. Mango, Architettura bizantina (1974) 144 e 151; F. W. Deichmann, Westliche Bautechnik im römischen und rhomäischen Osten, RM 86, 1979, 488-493 e passim 478-527; R. Krautheimer, Architettura paleocristiana e bizantina (1986) 279-280. 288-289. 291-292; F. De Maffei, Il palazzo di Qasr ibn-Wardan dopo gli scavi e i restauri, in: A. Jacobini - E. Zanini (ed.), Arte profana e arte sacra a Bisanzio (1996) 109-159; E. Russo, Una riflessione sull'episcopio di Parenzo, Bizantinistica serie seconda 2, 2000, 187-188 figg. 20-22.

${ }^{284}$ Il palazzo reca infatti due epigrafi, datate al 561 e al 564 (F. De Maffei in: Jacobini - Zanini [nota 285] 110 figg. 31-32). 
dei lavori è stata di recente estesa anche all'epoca di Giustino $\mathrm{II}^{285}$ : e sono certo che un'indagine attenta nell'ecumene bizantina porterebbe a individuare altre costruzioni analoghe in pianta alla chiesa romana. Come per S. Giovanni a porta Latina, le caratteristiche di S. Maria Antiqua indicano un'opera compiuta da uno o più architetti greco-costantinopolitani presenti a Roma ${ }^{286}$. Tutti i tentativi compiuti per posticipare la seconda fase cristiana dell'edificio ${ }^{287}$ cozzano non soltanto contro l'analisi formale delle pitture, ma pure contro la realtà archeologica dei ritrovamenti monetali ${ }^{288}$. Altra chiesa allora costruita a Roma è S. Maria in Cosmedin, alla metà del VI secolo in base all'analisi dell'edificio ${ }^{289}$. La dedica alla Madonna è probabile, com'è probabile la nuova consacrazione alla Vergine del battistero ariano a Ravenna intorno al $560^{290}$.

Abbiam ricordato il criterio dell'interazione uomo-ambiente, che attiene al rapporto tra maestranze venute dall'esterno, con la propria cultura e la propria tecnica, e l'ambiente e la realtà culturale in cui queste si trovano a operare. Esso va congiunto all'altro criterio dell'interazione uomouomo, vale a dire gli effetti e le conseguenze della collaborazione di maestranze d'origine, formazione culturale e tecnica, capacità diverse, che si verificano non soltanto quando

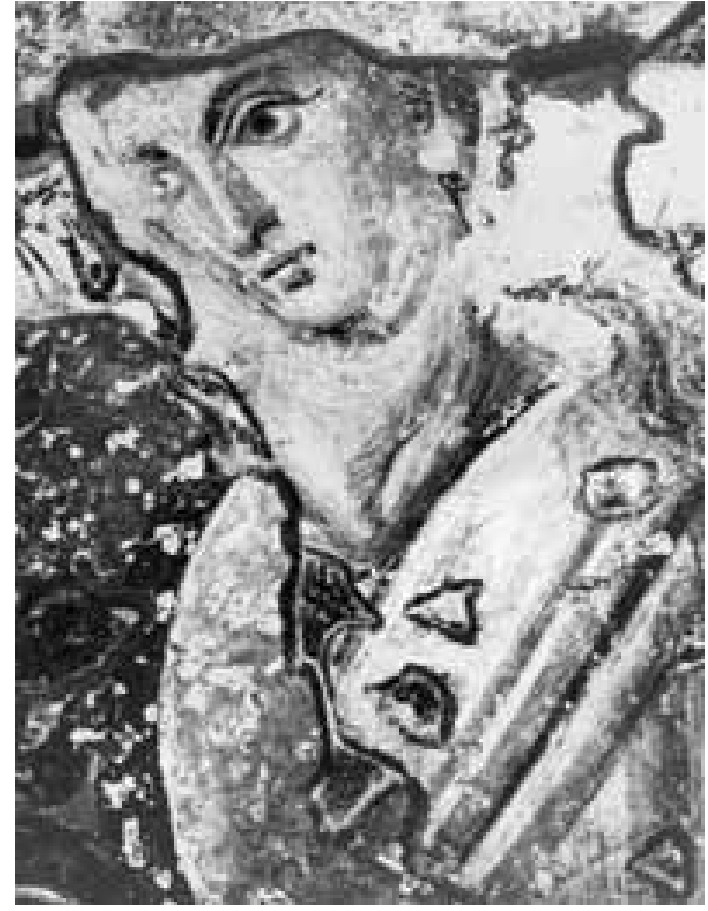

69 Roma, S. Maria Antiqua: angelo bello esse s'incontrano e cooperano alla realizzazione d'un medesimo monumento o complesso, ma pure quando maestranze locali non restan insensibili ad alcune delle novità apportate da maestranze straniere in occasione di realizzazioni precedenti ${ }^{291}$. I due criterii ci forniranno un sostegno sicuro per tentare di districarci nell'accidentato e contradditorio percorso della pittura, a partire dagli esiti formali così discordanti del ciclo di affreschi di S. Martino ai Monti ${ }^{292}$, dove una coeva realizza-

${ }^{285} \mathrm{Nel}$ palazzo è stata infatti di recente scoperta un’ulteriore epigrafe con la data del 572 (F. De Maffei in: Jacobini - Zanini [nota 285] 110-111 figg. 33-34).

${ }^{286}$ Invece B. Brenk, che non cita la bibl. riportata più sopra e menziona soltanto un’opera di H. C. Butler del 1929, e riferisce soltanto la data tradizionale del 564, non coglie il significato del confronto, e si limita a dire: »... von diesem Grundrisstypus in Rom nur gerade ein Pergamentplan zur Verfügung stand, aber das alleine verdient unsere Aufmerksamkeit. Wer kommt als Auftraggeber für diesen im lateinischen Westen einzigartigen Grundriss in Frage: die byzantinische Administration oder ein Papst? « (Brenk [nota 282] 1005): è evidente però che se pure gli operai, con la loro tecnica e i loro materiali, sono locali, la trasformazione d'un edificio classico non poteva giovarsi d'una semplice pianta, come se si dovesse costruire ex novo, e per ottenere un simile risultato eran presenti sul posto uno o più architetti non locali (giacché i locali avrebbero compiuto la trasformazione secondo canoni differenti). Sull'analisi delle murature vd. M. C. D’Alessandro, S. Maria Antiqua in: Materiali e tecniche (nota 17) 302-304.

${ }^{287}$ Da ultimo da Brenk (nota 282) 993-1019.

${ }^{288}$ Come si può chiaramente vedere in Russo (nota 274) 143-147.

${ }^{289}$ Krautheimer (nota 281) 291-304. 306-308 figg. 221. 224 tavv. 20-21 (a p. 307 Krautheimer nota: »vorremmo sottolineare che la tecnica di costruzione e forse il disegno generale dell'edificio, denotano influenze provenienti dalla parte greca dell'impero, specialmente dall'Italia meridionale«). Una datazione all'epoca di Teoderico o Atalarico, cioè ai primi decennii del VI sec., è invece in Giovenale (nota 238) spec. 332-333.

290 Agnellus, ed. Holder Egger (nota 276) 334; ed. Testi Rasponi (nota 276) 217-218 (cap. 86). Tuttavia Agnello parla soltanto di riconciliazione, così che non sappiamo con sicurezza quando avvenne la dedica alla Vergine: cf. F. W. Deichmann, Studi sulla Ravenna scomparsa, Felix Ravenna 103/04, 1972, 74-75; Deichmann (nota 2:1974) 252.

291 Russo (nota 8:2003) 109. Come fenomeno d'interazione uomo-uomo quello della prassi costruttiva o pittorica è più cogente dell'altro offerto nel campo della scultura dai manufatti d'imitazione a opera di maestranze locali, poiché per le sculture si deve di volta in volta distinguer l'imitazione da prodotti eseguiti in loco da maestranze non locali, straniere, nella fattispecie grecocostantinopolitane. È altresì evidente che l'interazione uomo-ambiente attiene piuttosto al rapporto tra maestranze venute dall'esterno, con la propria cultura e la propria tecnica, e l'ambiente e la realtà culturale in cui queste si trovano a operare.

${ }^{292}$ C. Davis Weyer - J. J. Emerick, The Early Sixth-Century Frescoes at S. Martino ai Monti in Rome, Römisches Jahrbuch für Kunstgeschichte 21, 1984, 1-60; M. Andaloro, Aggiornamento scientifico, in: G. Matthiae, Pittura romana del medioevo I (1987) 


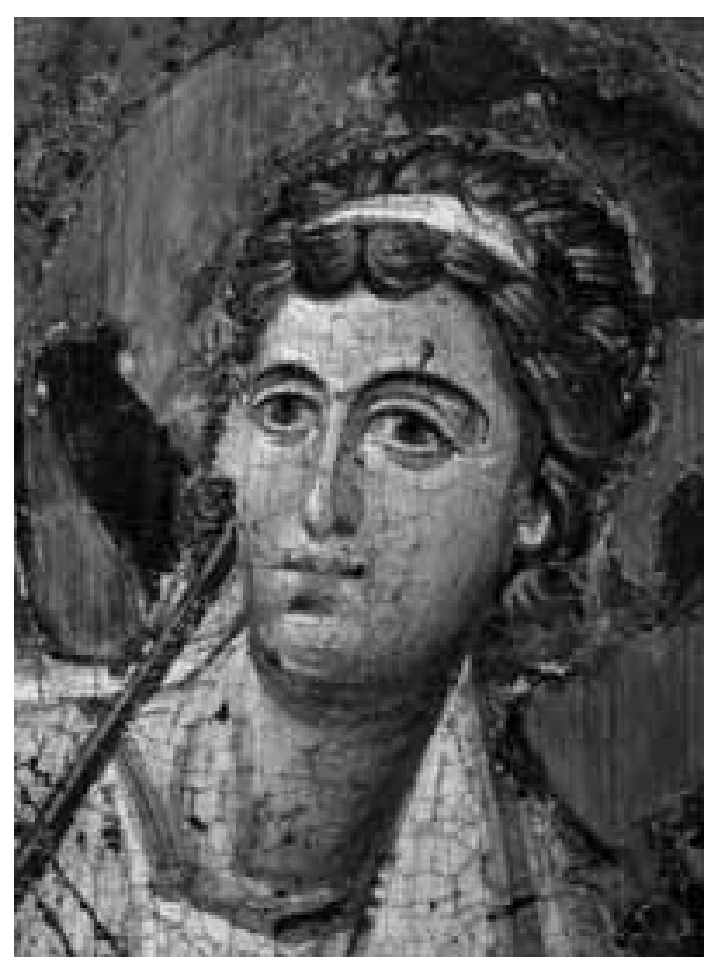

70 Roma, S. Maria in Trastevere: icona della Clemenza, volto dell'angelo destro

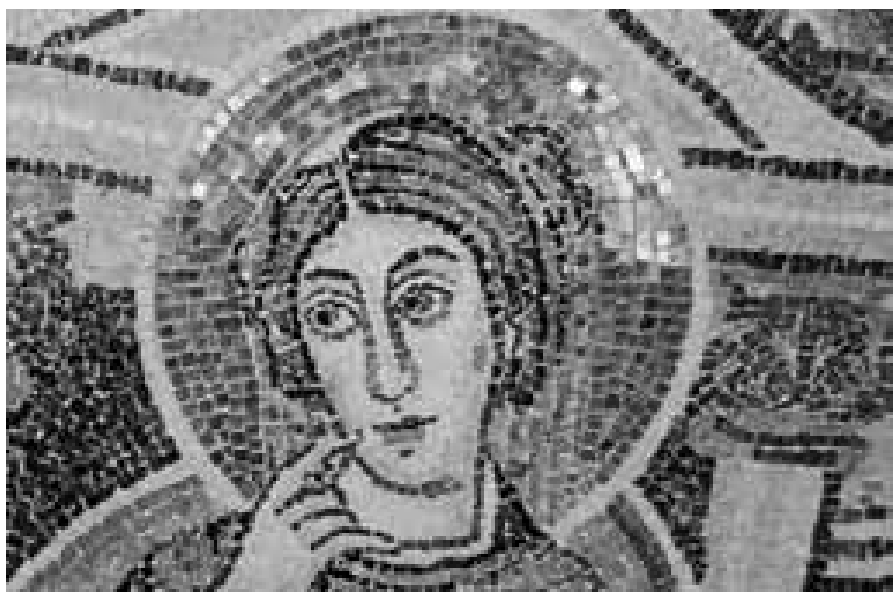

71 Parenzo, basilica eufrasiana: Madonna nell’Annunciazione

zione, ch'è stata collocata tra il 506 e il $514^{293}$, vede applicate 'modalità'294 diverse in uno stesso monumento, con pluralità di direzioni, »la plastico-volumetrica di sapore ravennate-teodoriciano nella pittura della lunetta; la pittorica, di formulazione compendiaria e tutta interna al flusso romano, nel brano dell'Annunciazione; e, infine, la rarefatta cifra di cui è intriso quel che rimane della figura di vescovo a mosaico, incline verso tratti estranei alla linea romana ${ }^{295}$. È quel che accade anche negli affreschi della prima fase, frammentarii, della basilica inferiore di S. Crisogono, datati entro la metà del VI secolo, e rappresentanti i Tre fanciulli ebrei nella fornace, la Madonna Regina in trono tra angeli, la croce gemmata con vela, dove convivono due tendenze, l'una »ancora debitrice alla tradizione classica «, l'altra che mostra »semplificazione delle linee« e "stilizzazione delle figure, secondo moduli orientali «296. È dunque evidente che per S. Martino ai Monti e per S. Crisogono si tratta di scelte intradirette e non eterodirette, per adoperar ancora la terminologia kitzingeriana, scelte legate e dipendenti dalla formazione, vale a dire dalla provenienza, romana o non romana, dei singoli pittori compresenti nelle botteghe che han realizzato gli affreschi.

Forti di quest'importante acquisizione, prenderemo in considerazione soltanto alcuni esempi della pittura a Roma nel VI secolo, sufficienti tuttavia a indicare con chiarezza la presenza di artefici greco-costantinopolitani: l'affresco della Traditio clavium nel cimitero di Commodilla ${ }^{297}$, del tempo di papa Giovanni I (523-

242-244 figg. 6-7; eadem, Pittura romana e pittura a Roma da Leone Magno a Giovanni VII, in: Committenti e produzione artistico-letteraria nell'alto medioevo occidentale. XXXIX Settimana di studio del Centro italiano di studi sull'alto medioevo, 4-10 aprile 1991 (1992) 580-584 figg. 6-9.

293 Davis Weyer - Emerick (nota 292) 54-57.

${ }^{294}$ Per usar il termine introdotto da E. Kitzinger, Byzantine Art in the Period between Justinian and Iconoclasm, in: Berichte zum XI. Internationalen Byzantinisten-Kongress (München 1958) 20-33.

295 M. Andaloro in: Committenti (nota 292) 583. Cf. Davis Weyer - Emerick (nota 292) 39-42.

296 A. Melograni, Le pitture del VI e VIII secolo nella basilica inferiore di S. Crisogono in Trastevere, RIA terza serie 13, 1990 (1991) 146-161 figg. 3-5. 11. 14. 17-19.

297 E. Russo, L'affresco di Turtura nel cimitero di Commodilla, l'icona di S. Maria in Trastevere e le più antiche feste della Madonna a Roma [prima parte], Bullettino dell'Istituto storico italiano per il medio evo e Archivio Muratoriano 88, 1979 (1981) 37 (523-526) (con la bibl. precedente); M. Andaloro in: Matthiae (nota 292) 234 (523-526); eadem in: Committenti (nota 292) 
526), l'affresco di Turtura nel medesimo cimitero ${ }^{298}$, degli stessi anni o súbito dopo, 527 o 528, l'affresco di Maria Regina nella parete palinsesto di S. Maria Antiqua ${ }^{299}$, degli anni '40 del VI secolo, quando il corpo di guardia ai piedi del Palatino fu cristianizzato, l'Angelo bello sulla stessa parete ${ }^{300}$, del tempo di Giustino II (565-578), quando l'edificio fu trasformato in chiesa con l'apertura dell'abside, l'icona di S. Maria Nova ${ }^{301}$, proveniente da S. Maria Antiqua e realizzata quando l'edificio fu trasformato in chiesa, l'icona di S. Maria in Trastevere ${ }^{302}$, pure dipinta in occasione della dedica alla Madonna dell'antico titulus Iuli in Trastevere, all'epoca di papa Giovanni III (561-574) o di papa Benedetto (575-579). E con un'avvertenza ben precisa: dobbiamo superare lo schema riduttivo che induce a vedere come caratteristica distintiva se non unica del mondo greco-costantinopolitano le espressioni che son state definite 'ellenistiche', quasi che soltanto in quest'apporto si debbano inquadrare quelle che son state chiamate le 'influenze' dell'arte bizantina.

Non si può certo dubitare della paternità greco-costantinopolitana dell'affresco con l'Angelo bello in S. Maria Antiqua, con la sua chiara valenza ‘ellenizzante’ (fig. 69) ${ }^{303}$. Vediamone dei confronti meditati, anche

585-587 fig. 10 (523-526); J. G. Deckers et. al., Die Katakombe »Commodilla«. Repertorium der Malereien (1994) 50-57 tavv. a colori 3-6 (VII sec.: Costante II o Costantino IV). Va detto che la datazione tarda al VII sec. è contraddetta, oltre che sul piano formale, anche dal punto di vista archeologico già dal 1936 (B. Bagatti, Il cimitero di Commodilla o dei martiri Felice ed Adautto presso la via Ostiense [1936] 105-106. 110; cf. Russo op. cit. 37).

298 Russo (nota 297) 35-49 (527 o 528) (con la bibl. precedente); J. Osborne, The Roman Catacombs in the Middle Ages, BSR 53, 1985, 300-302 tav. 16a (525 c.-535 c., cioè dopo il restauro del sito e prima delle guerre gotiche); M. Andaloro in: Matthiae (nota 292) 234. 249 (527-528 o anni vicini); eadem in: Committenti (nota 292) 587-590 fig. 14 (poco dopo la Traditio clavium); Deckers (nota 297) 61-65 tavv. 6-7 (VII sec.: Costante II o Costantino IV); M. Minasi, Le vicende conservative dell'affresco di Turtura nel cimitero di Commodilla, RACr 73, 1997, 65-94; eadem in: Aurea Roma (nota 6) 656-660 n. 373 (prima metà VI sec.); J. G. Deckers, Göttlicher Kaiser - Kaiserlicher Gott. Die Imperialisierung des Christentums im Spiegel der Kunst, in: F. A. Bauer - N. Zimmermann (edd.), Epochenwandel? Kunst und Kultur zwischen Antike und Mittelalter (2001) 15 fig. 36 (seconda metà del VII sec.); K. Schade, Frauen in der Spätantike - Status und Repräsentation (2003) 143. 245 tav. 20, 2 (»6.-7. Jh.«); E. Thunø, The Cult of Virgin, Icons and Relics in Early Medieval Rome, ActaAArtHist s. a. 17, 2003 (2004) 87 fig. 7 (»early sixthcentury «). Va detto che la datazione tarda al VII sec. è contraddetta, oltre che sul piano formale, anche dal punto di vista archeologico già dal 1936 (Bagatti [nota 297] 105-110; cf. Russo [nota 297] 35-49).

299 Russo (nota 297) 44 (anni ’40 del VI sec.) (con la bibl. precedente); Osborne (nota 298) 301-302 (metà VI sec., dopo Turtura); M. Andaloro in: Matthiae (nota 292) 249 (536-545); H. Belting, Bild und Kult. Eine Geschichte des Bildes vor dem Zeitalter der Kunst (1990) 142 (VI sec.); M. Andaloro in: Committenti (nota 292) 599. 612; eadem, Le icone a Roma in età preiconoclasta, in: Roma fra Oriente e Occidente. XLIX Settimana di studio del Centro italiano di studi sull'alto medioevo, 19-24 aprile 2001) (2002) 752-753 fig. 23 (età gota, prima della conquista bizantina); J. Osborne, Images of the Mother of God in Early Medieval Rome, in: Icon and Word. The Power of Images in Byzantium. Studies presented to Robin Cormack (2003) 138 (prima dell'Angelo bello); Brenk (nota 282) 993-1032 figg. 16-19 (crede che sia stata fatta entro il 578); Thunø (nota 298) 81 fig. 5 (VI sec.).

300 Russo (nota 297) 42-44; Russo (nota 274) 143-146. 146-147 (565-578) (con la bibl. precedente); C. Bertelli, Traccia allo studio delle fondazioni medievali dell'arte italiana, in: Storia dell'arte italiana II 1 (1983) fig. 1 (VI-VII sec.); P. J. Nordhagen, S. Maria Antiqua Revisited, Arte medievale 1, 1983, 50 (intorno al 600); idem, Roma og Konstantinopel. Maleri og Mosaikk (1983) 11 fig. 4 (VII sec.); M. Andaloro in: Matthiae (nota 292) 249-250 (565-578); P. J. Nordhagen, Italo-Byzantine Wall Painting of the Early Middle Ages: an 80-Year Old Enigma in Scholarship, in: Bisanzio, Roma e l'Italia nell'alto medioevo. XXXIV Settimana di studio del Centro italiano di studi sull'alto medioevo, 3-9 aprile 1986) (1988) 594 fig. 1 (VII sec., senza curarsi di citare o di controbattere chi la pensa diversamente anche sulla scorta di dati di fatto di natura archeologica); Belting (nota 299) 142-143 fig. 75 (VII sec.); M. Andaloro in: Committenti (nota 292) 600 fig. 22 (565-578); Russo (nota 279) 41 fig. 1 (565-578); M. Andaloro in: Roma fra Oriente (nota 299) figg. 17-18 tavv. 15-16 (565-578); eadem, Dal ritratto all’icona, in: Aa. Vv., Arte e iconografia a Roma. Dal tardo antico alla fine del medioevo (2002) 54 (565-578); Osborne (nota 299) 138-139 (»presumably 565-578«); Brenk (nota 282) 993-1032 figg. 17-19 (crede che sia stato fatto nel VII sec.).

301 Russo (nota 297) 50. 78; Russo (nota 274) 146 (565-578) (con la bibl. precedente); M. Andaloro in: Matthiae (nota 292$) 255$ (ultimo quarto del VI sec.); P. Amato, De vera effigiae Mariae. Antiche icone romane (1988) 18-24 fig. 1 a p. 19 (V sec.); Belting (nota 299) 83-86. 142-143 tav. 1 (VII sec.); M. Andaloro in: Aurea Roma (nota 6) 660-661 n. 375 fig. a p. 418 (ultimo quarto del VI sec.); K. Dotzer, Die Rekonstruktion der Marientafel aus Santa Maria Antiqua und ihre Aufstellung im Kirchenraum, Mitteilungen zur spätantiken Archäologie und byzantinischen Kunstgeschichte 2, 2000, 53-70 fig. 1; M. Andaloro in: Roma fra Oriente (nota 299) 744-745 figg. 2. 13 tavv. 15-16 (565-578); eadem in: Arte e iconografia (nota 300) 54 fig. 22 (avanzato VI sec., 565-578); Thunø (nota 298) 81 fig. 2 (VI sec.).

302 Russo (nota 297) 49-85; Russo (nota 274) 147-148 (Giovanni III, 561-574, o Benedetto, 575-579); M. Andaloro in: Matthiae (nota 292) 255 (fine VI sec.); Amato (nota 301) 26-32 fig. 1 a p. 27 (VI-VII sec.); Belting (nota 299) 143-148 tav. 2 (705-707); M. Andaloro in: Aurea Roma (nota 6) 662-663 n. 377 figg. a pp. 420-423 (avanzato VI sec.); eadem in: Roma fra Oriente (nota 299) 743-744 figg. 5. 15-16. 19 tavv. 12-14; eadem in: Arte e iconografia (nota 300) 53 figg. 24-27. 37; Osborne (nota 299) 137 (»probably Pope John VII«, 705-707); Thunø (nota 298) 81 fig. 3 (VI sec.).

${ }^{303}$ Vd. da ultimo M. Andaloro in: Roma fra Oriente (nota 299) 750; eadem in: Arte e iconografia (nota 300) 54. 


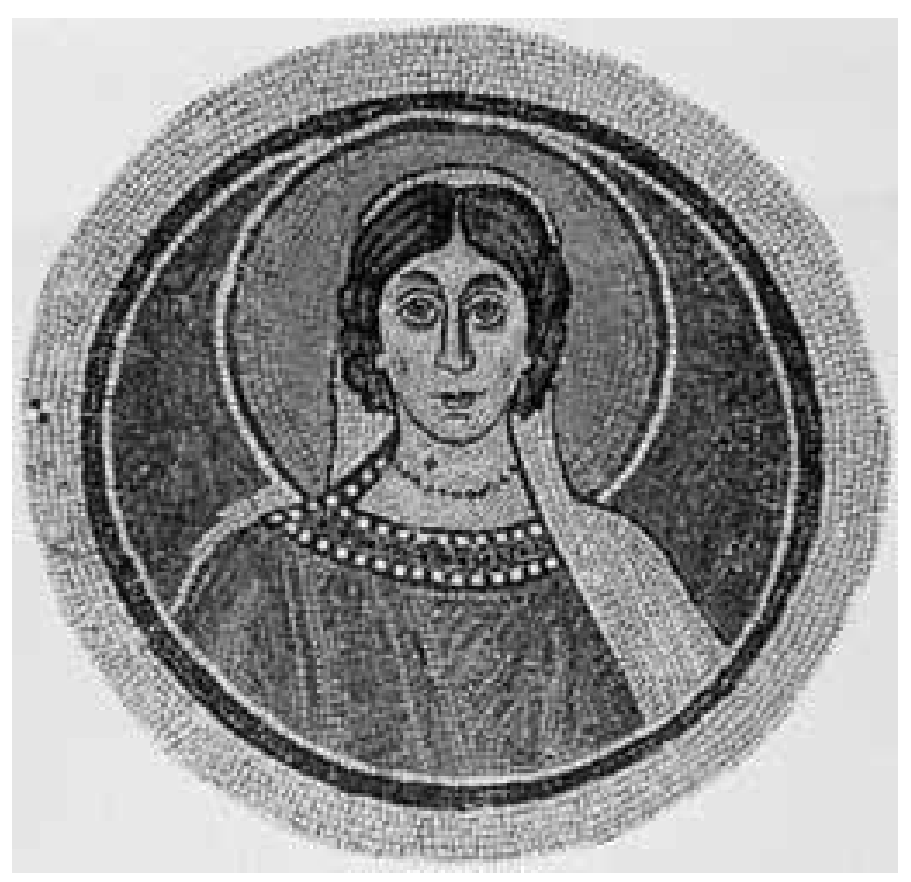

72 Parenzo, basilica eufrasiana: S. Agata se non ne verranno specificati analiticamente i punti di contatto: la Teodora del pannello di S. Vitale di Ravenna ${ }^{304}$, la Madonna della Fuga in Egitto a Castelseprio ${ }^{305}$, l'Angelo destro dell'icona di S. Maria in Trastevere (fig. 70) ${ }^{306}$, la Madonna nell'Annunciazione della basilica eufrasiana di Parenzo (fig. 71) ${ }^{307}$, la S. Agata dello stesso edificio (fig. 72) ${ }^{308}$, la testa di Teodora nel Castello Sforzesco di Milano ${ }^{309}$, opera prodotta a Costantinopoli, il busto femminile del Metropolitan di New York ${ }^{310}$, per la cui discussa datazione inclino al primo quarto del VI secolo, la testa maschile della Ny Carlsberg Glyptotek di Copenhagen (n. 3733) già in Collezione privata americana (fig. 73$)^{311}$, la testa femminile della Collezione George Ortiz di Ginevra, di probabile provenienza dalla Panfilia (fig. $74)^{312}$.

Ma bisogna ripercorrer tutta la pittura del secolo alla luce della presenza a Roma di maestranze greco-costantinopolitane, e senza limi-

304 Deichmann (nota 30:1969) tav. 365.

305 A. De Capitani d’Arzago in: G. P. Bognetti - G. Chierici - A. De Capitani d’Arzago, Santa Maria di Castelseprio (1948) tavv. 46. 47b; Russo (nota 279) 41 fig. 2. Il confronto è già in De Capitani d’Arzago op. cit. 680-681 tav. 81c.

306 Buona riproduzione in M. Andaloro in: Arte e iconografia (nota 300) fig. 26.

307 M. Prelog, Die Euphrasius-Basilika von Poreč (1986) tavv. 25. 27; idem, The Basilica of St. Euphrasius in Poreč (1994) tavv. 37. 40.

${ }^{308}$ Prelog (nota 307:1986) tav. 34; Prelog (nota 307:1994) tav. 43 in alto a sinistra.

309 S. Fuchs, Kunst der Ostgotenzeit (1944) 76 figg. 49. 51-52; W. F. Volbach - M. Hirmer, Arte paleocristiana (1958) 61 tav. 68 (metà VI sec.); D. Talbot Rice - M. Hirmer, Arte di Bisanzio (1959) 63 tav. 61 (530 circa); E. Alföldi Rosenbaum, Portrait Bust of a Young Lady of the Time of Justinian, MetrMusJ 1, 1968, 26 figg. 7. 9; S. Sande, Zur Porträtplastik des sechsten nachchristlichen Jahrhunderts, ActaAArtHist 6, 1975, 93-96 figg. 46-48 (molto probabilmente Teodora); J. D. Breckenridge in: Age of Spirituality. Late Antique and Early Christian Art, Third to Seventh Century (Catalogue of the Exhibition, New York, November 17, 1977 - February 12, 1978) (1979) 33 n. 27 (»Constantinople [?], about 530-540«); R. H. W. Stichel, Die römische Kaiserstatue am Ausgang der Antike (1982) 63-64 (sicuramente Teodora, 540 circa); P. Zanker in: Fittschen - Zanker III 37 (V sec.); Schade (nota 298) 80. 138. 227-228 tav. 67, 2-4 (VI sec.).

310 Alföldi Rosenbaum (nota 309) 19-35 figg. 1-5. 8. 10; Sande (nota 309) 95 nota 3 (inizio V sec.); Breckenridge (nota 309) 292-295 n. 272 (»Constantinople, 1st quarter 6th century«); E. Alföldi Rosenbaum in: J. İnan - E. Alföldi Rosenbaum, Römische und frühbyzantinische Porträtplastik aus der Türkei. Neue Funde (1979) 335-336 tav. 268 (giustinianeo); P. Zanker in: Fittschen - Zanker III 37 (V sec.); D. Stutzinger, Das Bronzebildnis einer spätantiken Kaiserin aus Balajnac im Museum von Niš, JbAC 29, 1986, 154-155 tav. 26a. c (intorno al 400); J. Meischner, Das Porträt der theodosianischen Epoche II (400 bis 460 n. Chr.), JdI 106, 1991, 403. 405 tav. 93, 2 (400-420); B. Kiilerich, Late Fourth Century Classicism in the Plastic Arts (1993) 121-123 fig. 68 (intorno al 400); J. Meischner, Bildnisse der Spätantike 193-500. Problemfelder. Die Privatporträts (2001) 113 figg. 323-324 (379-405); Schade (nota 298) 208-210 tav. 56, 1-3 (»aus der Zeit um 400«).

311 D. Kiang, A Late Antique-Early Byzantine Head, Pantheon 28, 1, 1970, 3-11 figg. 1-6 (orientale, circa 515-525); Sande (nota 309) 79-80 (primo quarto del VI sec.); H. Jucker, Marmorporträts aus dem römischen Ägypten, in: Das römisch-byzantinische Ägypten. Akten des internationalen Symposions, Trier, 26.-30. September 1978 (1983) 144-145 tavv. 12, 1-2; 13, 2 (Egitto, inizio del II sec.); P. Zanker in: Fittschen - Zanker III 37 (inizio VI sec.); J. Meischner, Theodosianische Porträts, in: Ritratto ufficiale e ritratto privato. Atti della II Conferenza internazionale sul ritratto romano, Roma, 26-30 settembre 1984 (1988) 375-376 fig. 1 (prima metà V sec., »mitteltheodosianisch«); eadem (nota 310:1991) 402 tav. 89, 3 (Alessandria, 420-440); eadem (nota 310:2001) 121 fig. 351 (430-455).

312 J. D. Breckenridge in: Age of Spirituality (nota 309) 295-296 n. 273 (»Asia Minor or Syria, mid-late 6th century«); D. Stutzinger in: Spätantike und frühes Christentum (Ausstellungskat. Frankfurt am Main, 16. Dezember 1983 - 11. März 1984) $474-475$ n. 77 (»angeblich aus dem Pamphylien, gegen die Mitte des 6. Jhs.«); G. Ortiz, Faszination der Antike. The George Ortiz Collection (1996) n. 251 (»angeblich aus Pamphylien«, »5. Jahrhundert n. Chr.?«); Meischner (nota 310:2001) 127 fig. 378 (455-476); Schade (nota 298) 138. 226-227 tavv. 66, 1-4; 67, 1 (VI sec.). 
tare la loro opera alla valenza puramente 'ellenizzante', dato che il panorama orientale era assai più articolato. Lo strato con la Maria Regina in S. Maria Antiqua (fig. 75) ci consente di confrontare la Madonna con la Madonna in trono nel mosaico absidale di Parenzo (fig. $76)^{313}$, con la già ricordata $\mathrm{S}$. Agata nel medesimo mosaico (fig. 72), con il Cristo dell'absidiola sud sempre di Parenzo (fig. 77) ${ }^{314}$, con le teste di Ariadne conservate rispettivamente nei Musei
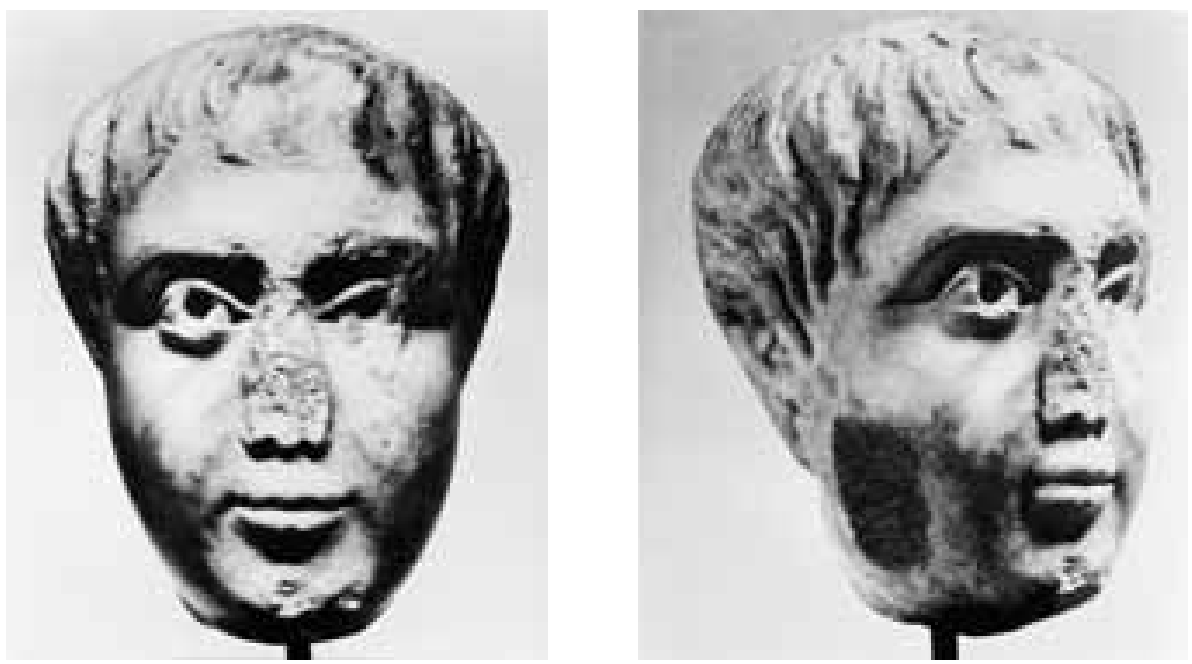

73 Copenhagen, Ny Carlsberg Glyptotek: testa maschile, già in Collezione privata americana Capitolini ${ }^{315}$, in S. Giovanni in Laterano ${ }^{316}$, al Louvre ${ }^{317}$, con la valva di dittico in avorio del Bargello di Firenze, sempre con Ariadne ${ }^{318}$, con la testa della Collezione Ortiz (fig. 74), con la testa femminile della Ny Carlsberg Glyptotek di Copenhagen (fig. 78) ${ }^{319}$. L’Angelo dell'affresco di Maria Regina (fig. 75) con il volto della defunta Turtura nell'af-

${ }^{313}$ Prelog (nota 307:1986) tavv. 37-38; Prelog (nota 307:1994) tavv. 5. 50. Il confronto è già in P. Toesca, Storia dell'arte italiana I 2: Il Medioevo (1914) 213 (rist. 1965, 223-224) fig. 130.

314 Prelog (nota 307:1986) tavv. 16. 20; Prelog (nota 307:1994) tavv. 24. 27.

315 Fuchs (nota 309) 71 figg. 47-48 (Amalasunta); Alföldi Rosenbaum (nota 309) 25-26 figg. 15-16 (Ariadne); Sande (nota 309) 67-68. 77-79 figg. 13-14; Stichel (nota 309) 59 tavv. 26. 28; P. Zanker in: Fittschen - Zanker III $36-38$ n. 39 tavv. 49-50 (»frühes 6. Jh. n. Chr.«); D. Stutzinger in: Spätantike (nota 312) 469-470 n. 73 (»Ende des 5., Anfang des 6. Jhs.«); A. Acconci in: Aurea Roma (nota 6) 581-582 n. 269 (marmo lunense; inizi VI sec.); Meischner (nota 310:2001) 132-133 (476-500; la Meischner crede a torto, in base ai luoghi di ritrovamento, che »... westliche Bedarf, wahrscheinlich auch die Tätigkeit westlicher Werkstätten nach 476 noch nicht erloschen waren, sondern unter ostgotischen Herrschaft fortleben «); Schade (nota 298) 80-81. 219-220 tavv. 63, 1; 64, 1-2 (um 500); B. Brenk, Zum Problem des Alterbildnisses in der spätantik-frühchristlichen Kunst, Arte medievale n. s. 2, 1, 2003, 12. 14 fig. 5 (prima del 515; eseguito a Costantinopoli e poi portato a Roma, molto probabilmente da Teoderico: ma queste idee di Brenk sono impossibili, dato che il marmo è lunense). Il confronto è già in M. Andaloro in: Committenti (nota 292) 612, e eadem in: Roma fra Oriente (nota 299) 752-753.

316 Alföldi Rosenbaum (nota 309) 25-26 figg. 11-12 (Ariadne); Sande (nota 309) 67-68. 77-79 figg. 9-11; Stichel (nota 309) 59 tav. 25; A. Acconci in: Aurea Roma (nota 6) 582 n. 270 (»Marmo, con tracce di policromia«; V-VI sec.); Schade (nota 298) 80-81. 220-222 tavv. 63, 3-4; 64, 3-4; Brenk (nota 315) 12-14 fig. 7 (prima del 515; eseguito a Costantinopoli e poi portato a Roma, molto probabilmente da Teoderico). Il confronto è già in M. Andaloro in: Committenti (nota 292) 612, e eadem in: Roma fra Oriente (nota 299) 752-753.

317 Alföldi Rosenbaum (nota 309) 25-26 figg. 13-14 (Ariadne); Sande (nota 309) 67-68. 77-79 fig. 12; J. D. Breckenridge in: Age of Spirituality (nota 309) 30-31 n. 24 (»Constantinople [?], about 500«); D. Stutzinger in: Spätantike (nota 312 ) 471 n. 74 (»Ende des 5., Anfang des 6. Jhs.«); J. R. Gaborit in: Byzance. L’art byzantin dans les collections publiques françaises (Catalogue de l'Exposition, Paris, 3 novembre 1992-1 ${ }^{\text {er }}$ février 1993) 38-39 n. 6 »(Marbre [Carrare?]. Constantinople ou Rome [?], début du VI siècle«; »il est bien certain que l'artiste qui l'a exécuté se réfère aux visages des portraits constantinopolitains et que, même si l'œuvre a été exécutée à Rome, c'est bien aux origines de l'art byzantin qu’il faut placer ce visage inquiétant«); A. Acconci in: Aurea Roma (nota 6) 582-583 n. 271 (»Marmo, [Carrara?]«; V-VI sec.); Schade (nota 298) 80-81. 223-224 tav. 63, 2; Brenk (nota 305) 112. 114 fig. 6 (prima del 515; eseguito a Costantinopoli e poi portato a Roma, molto probabilmente da Teoderico: ma queste idee di Brenk sarebbero impossibili, se fosse accertato che il marmo è il lunense). Il confronto è già in M. Andaloro in: Roma fra Oriente (nota 299) 752-753.

${ }^{318}$ Fuchs (nota 309) 70 figg. 44-46 (Amalasunta); Talbot Rice - Hirmer (nota 309) 50 tav. 21 (Ariadne; Costantinopoli; 500 circa); Alföldi Rosenbaum (nota 309) 27 fig. 21; W. F. Volbach, Elfenbeinarbeiten der Spätantike und des frühen Mittelalters ${ }^{3}$ (1976) n. 51 (»um 500«); E. La Rocca, Divina ispirazione, in: Aurea Roma (nota 6) 31 fig. 39 (Pulcheria, V sec.); A. McClanan, Representations of Early Byzantine Empresses. Image and Empire (2002) 168-173 fig. 7.7 (Sofia, moglie di Giustino II); Schade (nota 298) 110. 147. 244 tav. 13, 4 (»um 500«). Il confronto è già in M. Andaloro in: Roma fra Oriente (nota 299) 752-753 fig. 24.

319 V. Poulsen, Copenhagen, Ny Carlsberg Glyptotek. Les portraits romains II (1974) 207-208 n. 213 tavv. 349 - 350 (collegata con il c.d. Leone I dello stesso Museo e con il busto del Metropolitan; fine del V sec.); F. Johansen, Ny Carlsberg Glyptotek. Roman 

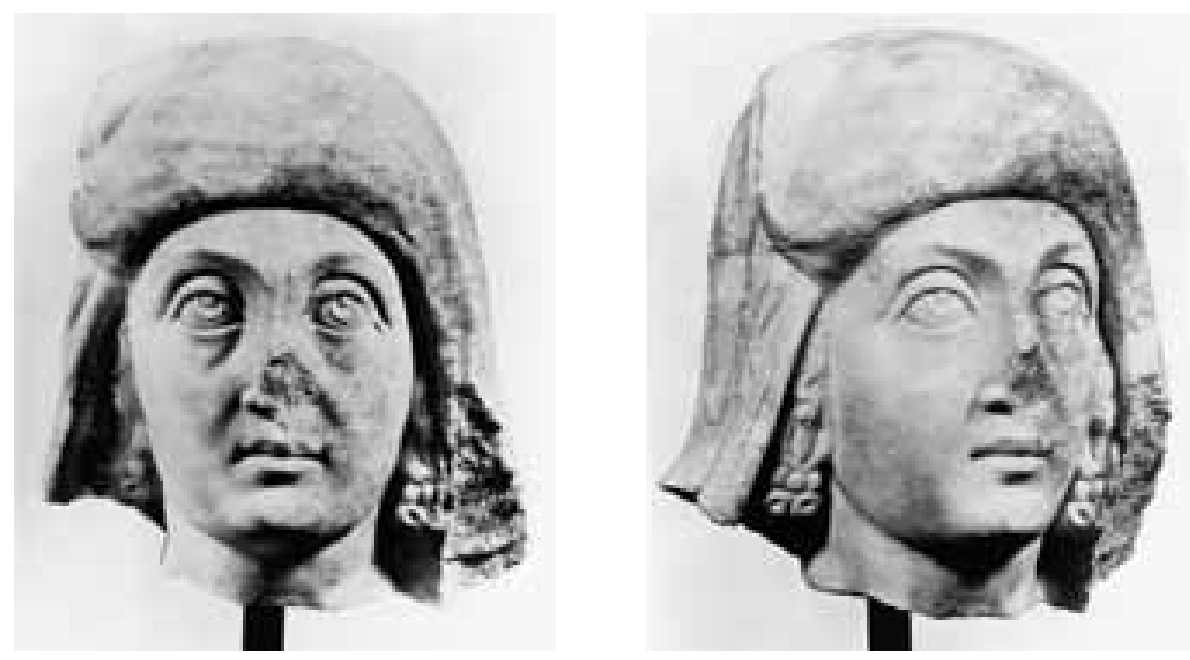

fresco di Commodilla (fig. 79) ${ }^{320}$, con il Cristo dell'arco nord-est della cappella arcivescovile di Ravenna ${ }^{321}$, 494-520, con l'Angelo sinistro dell'icona di S. Maria in Trastevere (fig. 80) ${ }^{322}$, con la testa della Collezione Ortiz (fig. 74), con la testa femminile di Copenhagen (fig. 78). E il Bambino dell'affresco di Maria Regina (fig. 75) con il Bambino del mosaico absidale di Parenzo (fig. $76)^{323}$, con il Bambino del mosaico absidale della Panaghia Kanakariá di Lythrankomi a Cipro (fig. 81 ${ }^{324}$, ritenuto del terzo decennio del VI secolo, con l'Arcangelo nord del medesimo mosaico (fig. 82) ${ }^{325}$, con la testa femminile di Copenhagen (fig. 78). Ora M. Andaloro, dopo una lunga militanza a favore del carattere bizantino dell'affresco di Maria Regina, con attribuzione agli anni '40 del VI secolo, dopo la conquista bizantina, dal 1991 ha espresso i suoi dubbi al riguardo ${ }^{326}$, e ritiene l'opera eseguita in età gota, e la Madonna è la »trasposizione dei ritratti femminili« in marmo di Ariadne a Roma e a Parigi e "pendant in pittura della temperie espressa dall'avorio del Bargello ${ }^{327}$. Ma questi giusti confronti, lungi dall’indebolire la paternità greco-costantinopolitana dell'opera, la rafforzano, in quanto le tre teste e l'avorio - cui vanno aggiunti il frammento di testa maschile dal Palatino oggi nel Museo dell'Alto Medio Evo ${ }^{328}$, il frammento di testa nella Glyptothek di Monaco di Baviera, già in Collezione privata svizzera ${ }^{329}$, la valva di dittico con Ariadne nel Kunsthistorisches Museum di Vienna ${ }^{330}$ - son stati realizzati o a Costantinopoli o a Roma da maestranze comunque sicuramente greco-costantinopolitane.

Egualmente può dirsi per una parte almeno dell'affresco di Turtura, nonostante sia stato notato per esso un »sostanziale carattere romano« e »la recezione dell'elemento ravennate« sarebbe limitata alla »sfera tipo-

Portraits Catalogue III (1995) 202 n. 89 figg. a pp. 202-203 (390-400); Meischner (nota 310:2001) 132-133 fig. 383 (476-500); Schade (nota 298) 210-211 tav. 57, 1-4 (»erstes Viertel des 5. Jahrhunderts«).

320 Buona riproduzione in Deckers (nota 297) tav. 7.

${ }^{321}$ Deichmann (nota 30:1969) tav. 227.

322 Buona riproduzione in M. Andaloro in: Arte e iconografia (nota 300) fig. 25.

${ }^{323}$ Prelog (nota 307:1986) tavv. 37-38. 45; Prelog (nota 307:1994) tavv. 5. 50.

${ }^{324}$ A. H. S. Megaw - E. J. W. Hawkins, The Church of the Panagia Kanakariá at Lythrankomi in Cyprus. Its Mosaics and Frescoes (1977) tavv. 78-79. 85. 88-89. 135-136. A pp. 137-140 la chiesa è datata alla terza decade del VI sec.

${ }^{325}$ Ibidem tavv. 73-76. 134.

${ }^{326}$ M. Andaloro in: Committenti (nota 292) 599. 612; eadem in: Roma fra Oriente (nota 299) 752-753.

${ }^{327}$ M. Andaloro in: Roma fra Oriente (nota 299) 752-753 fig. 24.

${ }^{328}$ A. P. Ruggiu Zaccaria, Una testa tardo-antica nel Museo dell'alto medioevo in Roma, in: Alto Medioevo 1 (1967) 35-54 figg. 1-3 (marmo lunense; Roma; Giustino II); Sande (nota 309) 66 fig. 4; M. S. Arena in: M. S. Arena - L. Paroli, Museo dell’Alto Medioevo, Roma (1993) 11-15 fig. 9 (Costantinopoli, Anastasio I); A. Acconci in: Aurea Roma (nota 6) 583 n. 272 (marmo lunense; inizi del VI sec.).

${ }^{329}$ S. Sande, Ein Porträt des 6. Jahrhunderts n. Chr., AA 1992, 599-607 figg. 1-6 (prima metà VI sec.); R. Wünsche in: Rom und Byzanz. Schatzkammerstücke aus bayerischen Sammlungen (Katalog zur Ausstellung, München, 20. Oktober 1998-14. Februar 1999) 72-75 n. 2 (»Römisch [?], I. Hälfte des 6. Jahrhunderts«); idem in: Aurea Roma (nota 6) 583-584 n. 273 (traduzione del testo del 1998).

${ }^{330}$ Fuchs (nota 309) 70 fig. 43 (Amalasunta); Volbach (nota 318) n. 52 (»Konstantinopel, um 500«); J. D. Breckenridge in: Age of Spirituality (nota 309) 31-33 n. 25 (»Constantinople, about 500-520«); E. La Rocca in: Aurea Roma (nota 6) 31 (Licinia Eudoxia, V sec.); K. S. Painter in: Aurea Roma (nota 6) 580-581 n. 268 (Ariadne, Costantinopoli, circa 500-520); McClanan (nota 318) 168-173 fig. 7.6 (Sofia, moglie di Giustino II); Schade (nota 298) 110. 144. 147. 244 tav. 13, 3 (»um 500«). 
logica «331. Ribadisco che a mani di pittori romani di quella bottega appartengon le teste dei Ss. Felice e soprattutto Adautto ${ }^{332}$, mentre a pennelli non romani si devono i volti della Madonna (fig. 83) ${ }^{333}$ e di Turtura (fig. 79$)^{334}$. Si confronti infatti il volto della Madonna (fig. 83) di Turtura con la Madonna al centro del mosaico absidale di Parenzo (fig. 76) ${ }^{335}$, con la S. Felicita della cappella arcivescovile di Ravenna ${ }^{336}$, con la seconda dama a sinistra di Teodora nel pannello di S. Vitale ${ }^{337}$, con il ritratto maschile - c.d. Leone I o Anastasio - della Ny Carlsberg Glyptotek di Copenhagen (fig. $84)^{338}$, con la testa di alabastro del Louvre (fig. 85) ${ }^{339}$, con la testa della Ny Carlsberg Glyptotek, già in Collezione privata americana (fig. 73), con la testa nella Collezione Ortiz (fig. 74): si tratta d'un insieme di opere non romane. $\mathrm{O}$ il volto di Turtura (fig. 79): da paragonare all'Angelo dell'affresco di Maria Regina a S. Maria Antiqua (fig. 75), all'Angelo bello della stessa chiesa (fig. 69), alla Teodora di S. Vitale, alla testa femminile di Copenhagen (fig. 78), al busto del Metropolitan di New York, all'Ariadne dei Musei Capitolini, all'Ariadne di S. Giovanni in Laterano, all'Ariadne della valva del Bargello, alla testa di alabastro del Louvre (fig. 85), alla testa della Collezione Ortiz (fig. 74). Un abisso sul

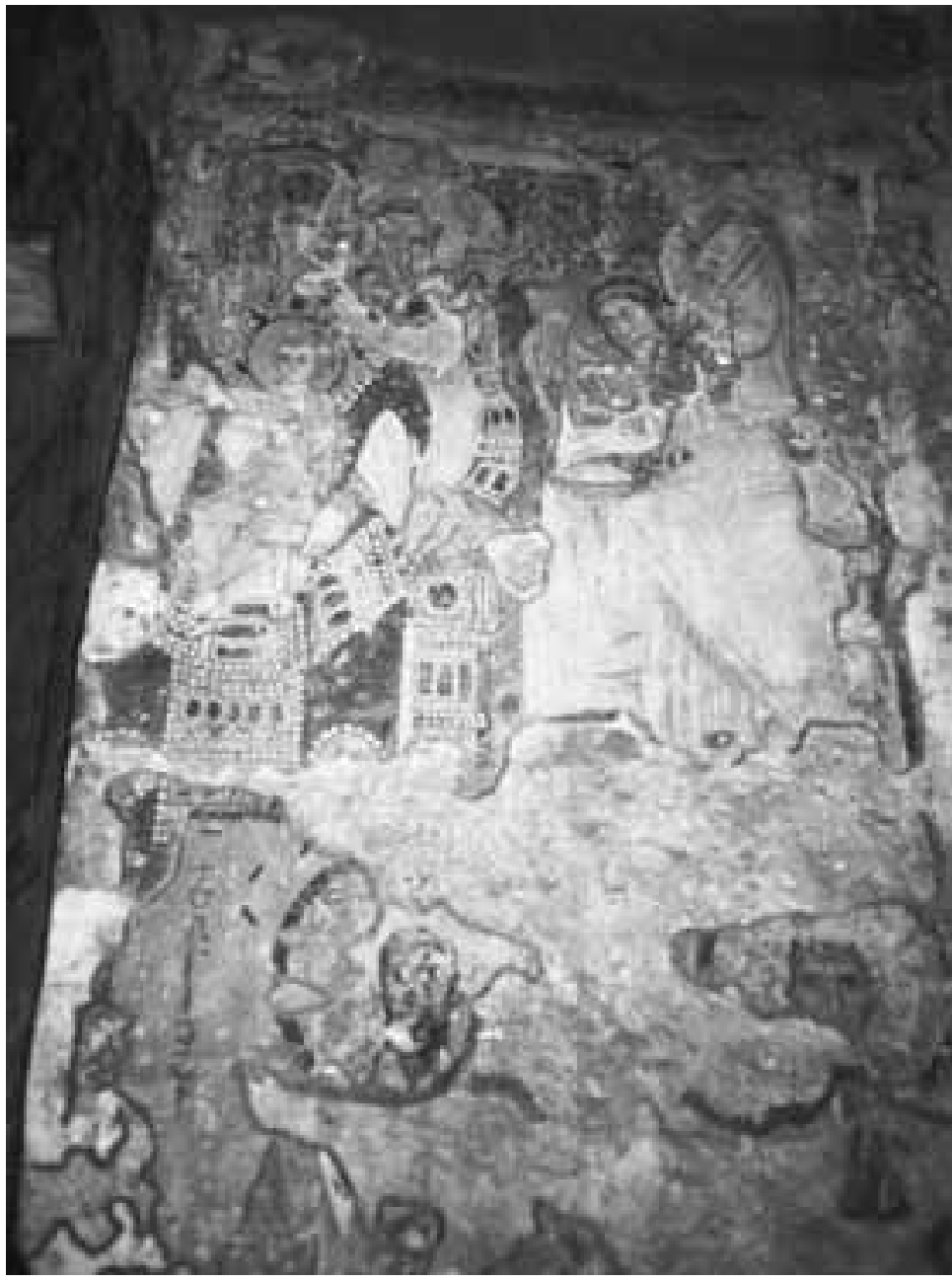

75 Roma, S. Maria Antiqua: parete palinsesto

piano formale divide queste due teste da un'opera coeva sicuramente romana come il mosaico dei Ss. Cosma e Damiano ${ }^{340}$.

\footnotetext{
${ }_{331}$ M. Andaloro in: Committenti (nota 292) 588. 589.

332 Come avevo detto in Russo (nota 297) 46.

333 Buona riproduzione in Bagatti (nota 297) fig. 100.

${ }^{334}$ Non chiaro, e comunque non condivisibile è il giudizio sul ritratto di Turtura espresso da C. Bertelli, La Madonna di Santa Maria in Trastevere (1961) 176 nota 76, secondo cui »esso è dipinto con una maniera più fluida e scorrevole, che ha fatto appunto pensare a un'influenza sellenistica $<$, mentre si tratta piuttosto di una intenzione naturalistica che apparenta questa figura, uscita da un pennello alquanto modesto, a quelle dei virtuosi del `naturalismo ellenistico«.

335 Il confronto è già in Toesca (nota 313) 211 (rist. 1965, 221-222) fig. 129. Cf. Russo (nota 297) 39.

336 Deichmann (nota 30:1969) tav. 239. Il confronto è già in Russo (nota 297) 46.

337 Deichmann (nota 30:1969) tav. 363. Il confronto è già in Russo (nota 297) 46-47.

338 Ruggiu Zaccaria (nota 328) 35-54 figg. 8-9 (Roma, Giustino II); Poulsen (nota 319) 197-198 n. 204 tavv. 333-334 (Leone I [?], 457-474; Poulsen giustamente per primo nel 1956 collega questo ritratto con il frammento di volto del Museo dell'Alto Medioevo); Sande (nota 309) 65-66. 76-77 figg. 1-3 (»kurz vor oder nach 500«, probabilmente Anastasio); Stichel (nota 309) 59-60 tavv. 27. 29 (Anastasio I); Sande (nota 329) 602 fig. 10 (inizio VI sec.); Johansen (nota 319) 182 n. 80 figg. a pp. 182-183 (»around $500 \ll)$.

${ }^{339}$ Sande (nota 309) 66-67. 75-76 figg. 5-7 (»aus der Zeit um 500«).

340 Non condivido infatti l'interpretazione di E. Kitzinger, Byzantine Art in the Making (1977) 92-94. 99-101, che vede nel mosaico dei Ss. Cosma e Damiano un'espressione di quella che lo studioso ha chiamato la sintesi giustinianea, e mi riconosco piuttosto nella valutazione di M. Andaloro in: Committenti (nota 292) 590-596; eadem in: M. Andaloro - S. Romano, L'immagine nell'abside, in: Arte e iconografia (nota 300) 78-80.
} 


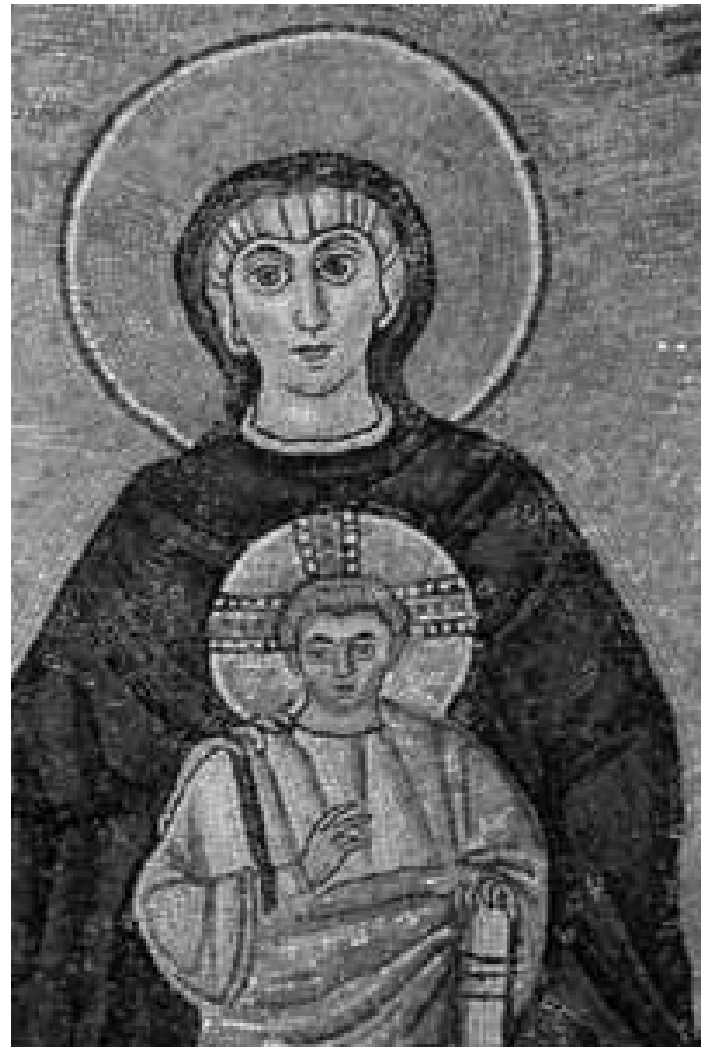

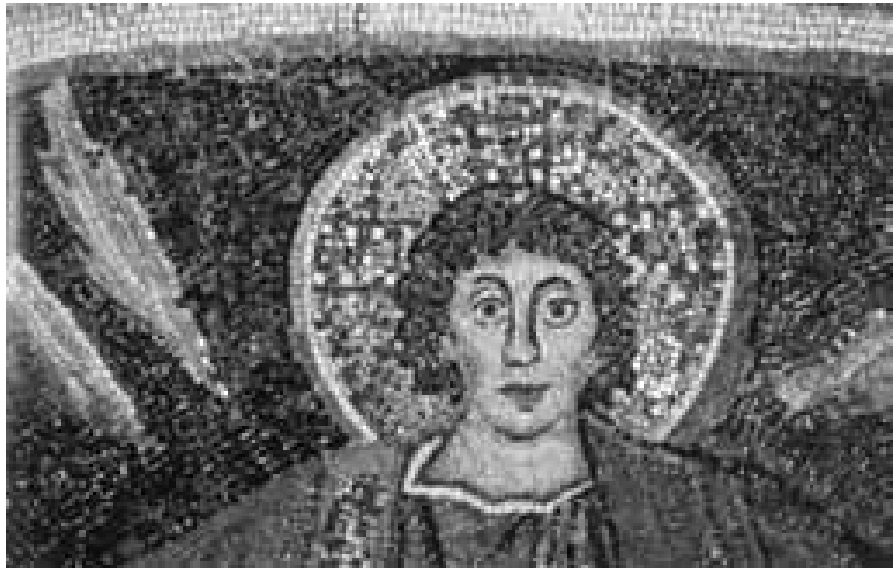

77 Parenzo, basilica eufrasiana: Cristo nell’absidiola sud

76 Parenzo, basilica eufrasiana:

Madonna in trono col Bambino nel mosaico absidale

Nella stessa Traditio clavium di Commodilla - sebbene sia stata giudicata »Elementare negli esiti, povera di indicazioni, ... quale prodotto di botteghe, il cui compito principale sembrerebbe consistere nel tradurre visivamente uno schema iconografico dato ${ }^{341}$ - si staccano dagli altri, e rivelan un'esecuzione di greco-co-

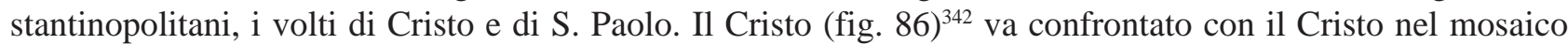
dell'absidiola nord di Parenzo (fig. 87) ${ }^{343}$, con S. Gervasio (fig. 88) ${ }^{344}$ e con S. Giovanni (fig. 89) ${ }^{345}$ nei medaglioni di S. Vitale; S. Paolo (fig. 90) ${ }^{346}$ rivela singolari affinità con S. Matteo (fig. 91) ${ }^{347}$ e con S. Bartolomeo $^{348}$ nella Panaghia Kanakariá di Cipro, con un Profeta di S. Apollinare Nuovo ${ }^{349}$ e con S. Taddeo in uno dei medaglioni di S. Vitale (fig. 92) 350 .

Questa rete di confronti permette di riportare all'àmbito greco-costantinopolitano non soltanto la fluida pennellata 'ellenistica' ma pure la netta definizione geometrica e l'astrazione lineare. Del resto anche nel campo dei dittici in avorio dobbiam guardare decisamente verso Costantinopoli, e soltanto per quello di Oreste del 530, conservato nel Victoria and Albert Museum di Londra (fig. 93) ${ }^{351}$, possiamo esser certi che si

${ }^{341}$ M. Andaloro in: Committenti (nota 292) 587.

342 Buona riproduzione in Deckers (nota 297) tav. a colori 4.

343 Prelog (nota 307:1986) tav. 15; Prelog (nota 307:1994) tavv. 23. 25.

${ }^{344}$ La basilica di San Vitale (nota 30) fig. 605.

${ }^{345}$ P. Racagni, Note tecniche sulla realizzazione del mosaico dell'arco presbiteriale di San Vitale, in: Mosaici a S. Vitale e altri restauri. Il restauro in situ di mosaici parietali. Atti del Convegno nazionale, Ravenna, 1-3 ottobre 1990 (1992) figg. 10-13 e fig. a p. 68.

346 Buona riproduzione in Deckers (nota 297) tav. a colori 5b.

347 Megaw - Hawkins (nota 324) tavv. 49 in alto; 59-60. 139.

${ }^{348}$ Ibidem tavv. 48 in basso; 69-70.

349 Deichmann (nota 30:1969) tav. 139.

${ }^{350}$ La basilica di San Vitale (nota 30) fig. 604.

351 Volbach (nota 318) n. 31 (»Rom 530«; »Das Diptychon stimmt mit dem des Clementinus überein.«); D. Stutzinger in: Spätantike (nota 312) 652-654 n. 231 (530; il dittico è »dem Diptychon des Clementinus aus dem Jahre 513, kopiert«). 


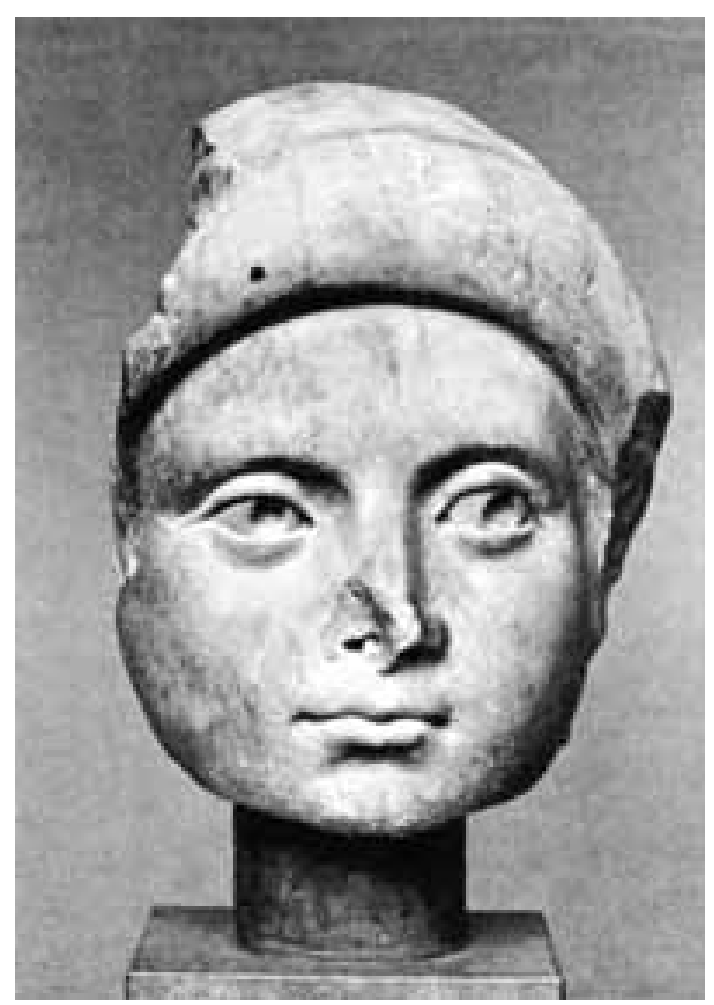

78 Copenhagen, Ny Carlsberg Glyptotek: testa femminile

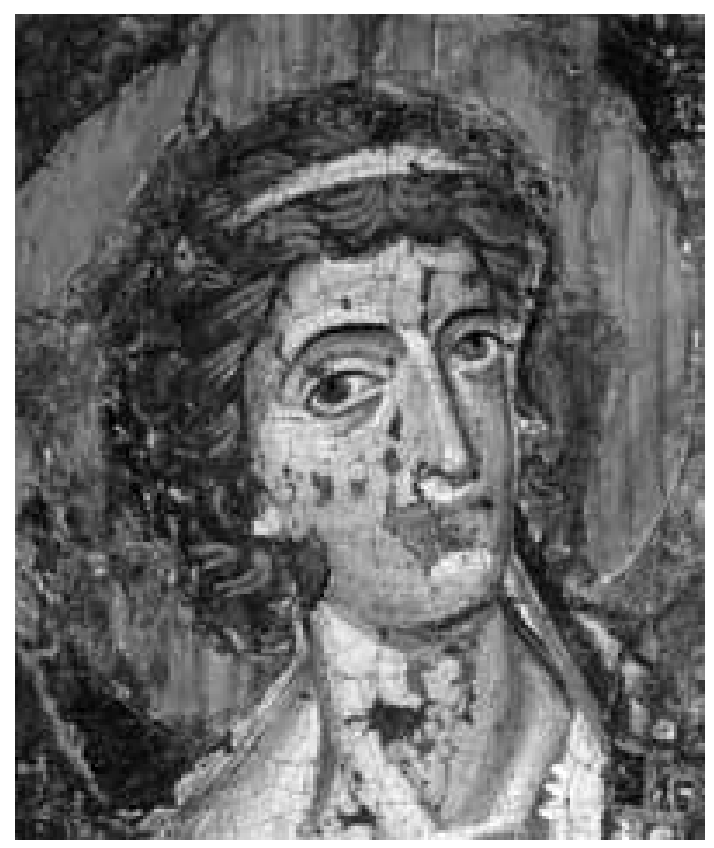

80 Roma, S. Maria in Trastevere: icona della Clemenza, volto dell'angelo sinistro

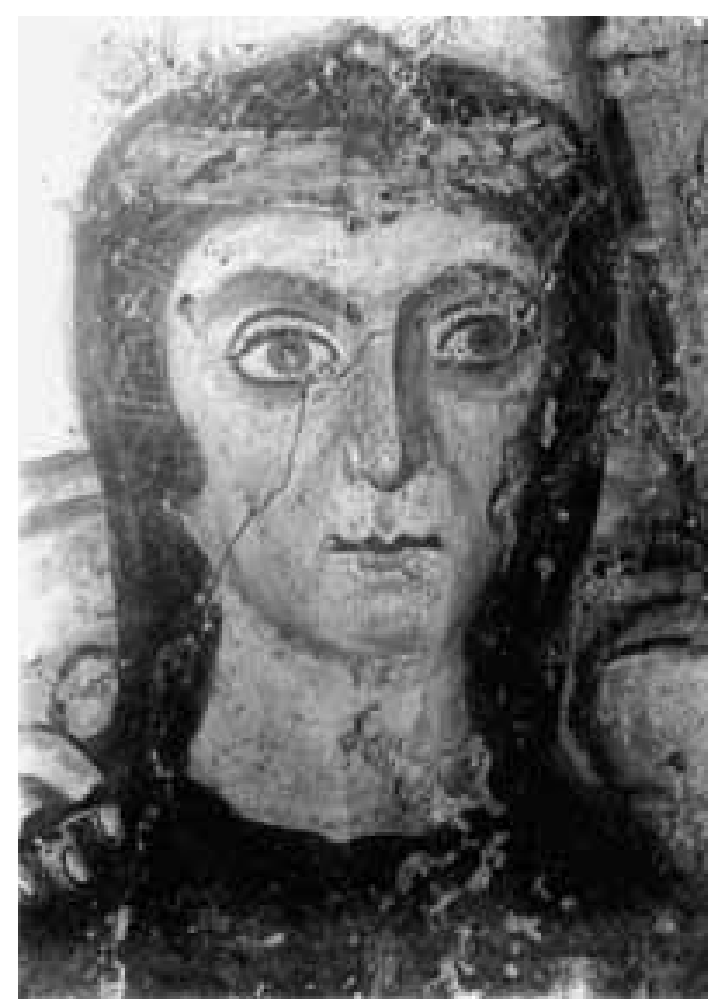

79 Roma, catacomba di Commodilla: affresco di Turtura, volto di Turtura

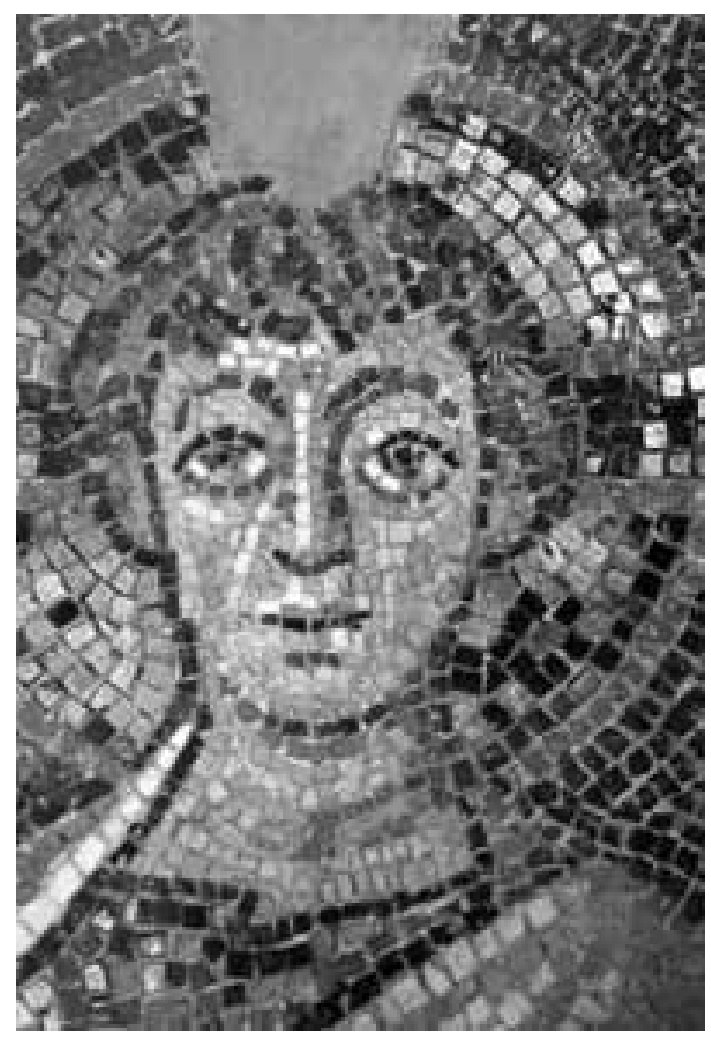

81 Lythrankomi (Cipro), Panaghia Kanakariá: il Bambino nel mosaico absidale 


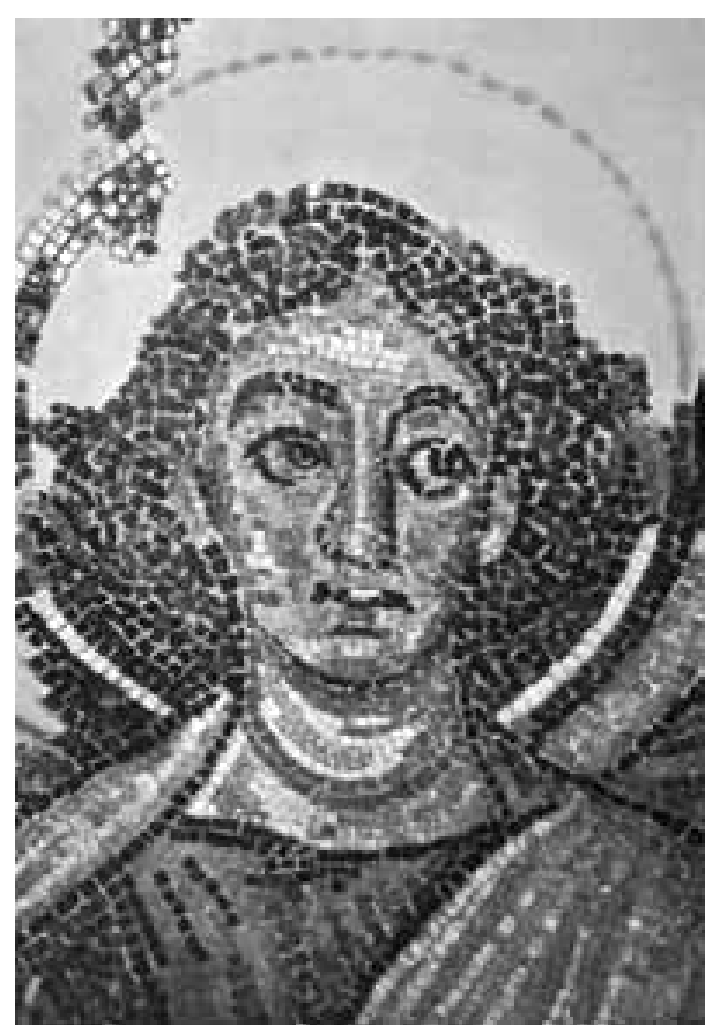

82 Lythrankomi (Cipro), Panaghia Kanakariá: l'arcangelo nord nel mosaico absidale

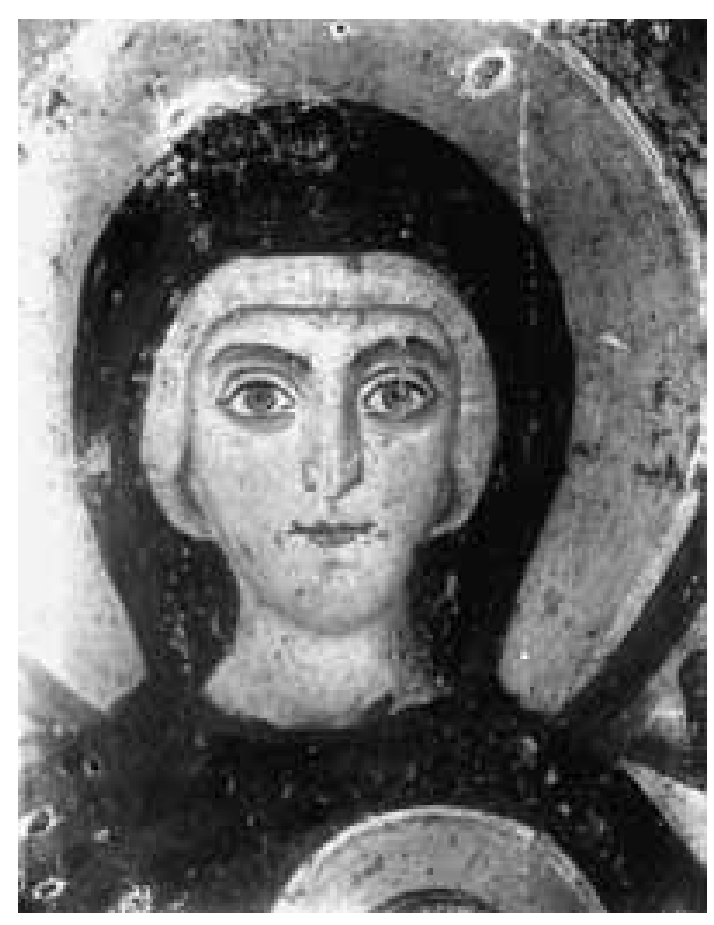

83 Roma, catacomba di Commodilla: affresco di Turtura, volto della Madonna

tratti d'una lavorazione a Roma da parte di artefici romani, i quali peraltro presero come modello il dittico costantinopolitano di Clementino del $513^{352}$.

Quanto è stato notato per gli affreschi si riscontra anche nelle icone di Roma, dove l'Andaloro ha già rilevato forte e "sempre più solido il quoziente della temperie ellenizzante di timbro costantinopolitano « nella Madonna dell'icona di S. Maria Nova, nel Bambino e nell'Angelo destro dell'icona di S. Maria in Trasteve$\mathrm{re}^{353}$. Si confrontino infatti il volto della Madonna nell'icona di S. Maria Nova (fig. 94) ${ }^{354}$ con la Teodora nel mosaico di S. Vitale, con la Madonna nell'icona di S. Maria in Trastevere (fig. 95) ${ }^{355}$, con la Teodora del Castello Sforzesco, con il busto del Metropolitan; o l'Angelo destro dell'icona di S. Maria in Trastevere (fig. 70) con l'Angelo bello di S. Maria Antiqua (fig. 69), con l'Arcangelo nord del mosaico absidale della Panaghia Kanakariá a Lythrankomi (fig. 82), con la testa della Ny Carlsberg Glyptotek già in Collezione privata americana (fig. 73), con la testa della Collezione Ortiz (fig. 74). Ma pure il volto della Madonna dell'icona di S. Maria in Trastevere (fig. 95) trova confronti convincenti con la Madonna dell'icona di S. Maria Nova (fig. 94), con la Teodora del mosaico di S. Vitale, con la Madonna dell'affresco di Turtura nel cimitero di Commodilla (fig. 83), con il busto del Metropolitan. E il volto dello stesso Angelo sinistro dell'icona di Trastevere (fig. 80) è da metter in relazione con l'Angelo dell'affresco di Maria Regina in S. Maria Antiqua (fig. 75). Quest'assieme di rimandi incrociati ci fà comprender meglio - nel momento in cui evochiamo la presenza di maestranze non romane a Roma e la valutiamo attraverso i due criterii dell'interazione uomoambiente e dell'interazione uomo-uomo - la portata del fenomeno e del ruolo giocato dai greco-costantinopolitani nell'Urbe nel VI secolo. Per le icone inoltre non si potrà non tener conto del peso della committenza,

\footnotetext{
${ }^{352}$ Volbach (nota 318) n. 15 (»Konstantinopel 513«); J. C. Anderson in: Age of Spirituality (nota 309) 48-50 n. 48; S. C. Bean in: Aurea Roma (nota 6) 34-35 n. 34.

353 M. Andaloro, La datazione della tavola di S. Maria in Trastevere, RIA n.s. 19/20, 1972/73 (1975) 163. 182; eadem in: Roma fra Oriente (nota 299) 750. La studiosa include nell'elenco il Bambino dell'icona del Pantheon (609), che noi non prendiamo in considerazione in questa sede.

354 Buona riproduzione in M. Andaloro in: Arte e iconografia (nota 300) fig. 22.

355 Buona riproduzione ibidem fig. 27.
} 
84 Copenhagen, Ny Carlsberg Glyptotek: ritratto maschile, c. d. Leone I o Anastasio
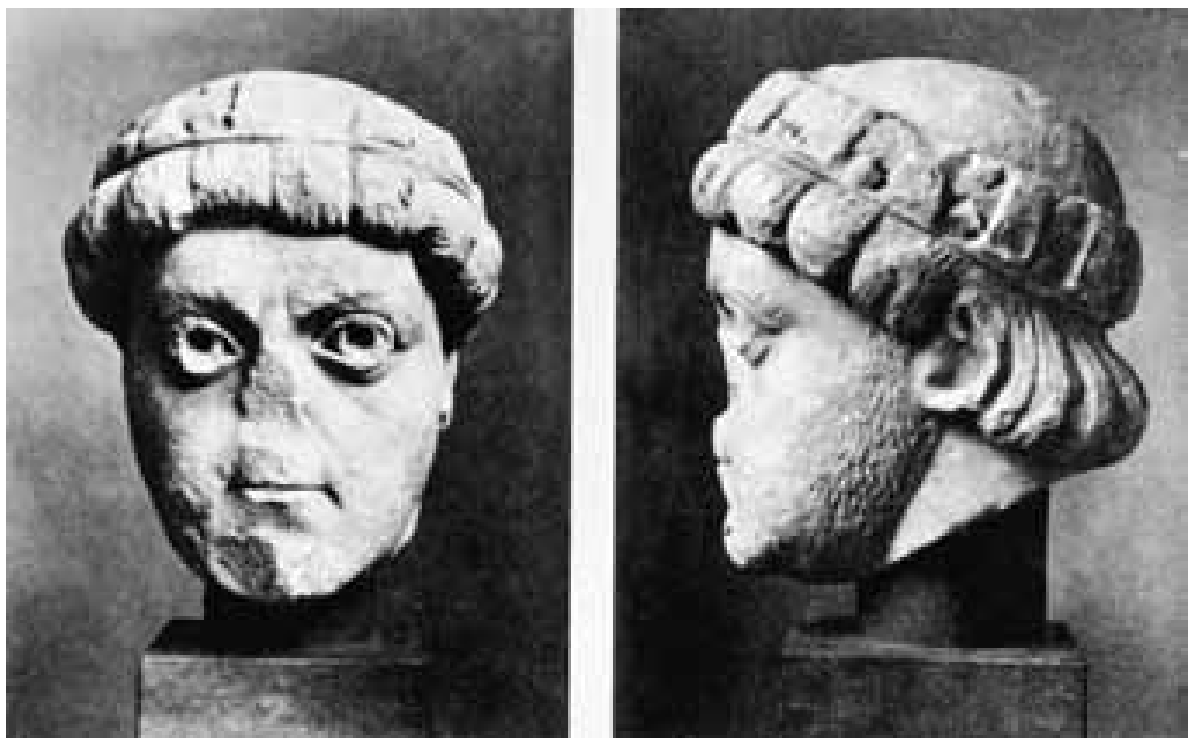

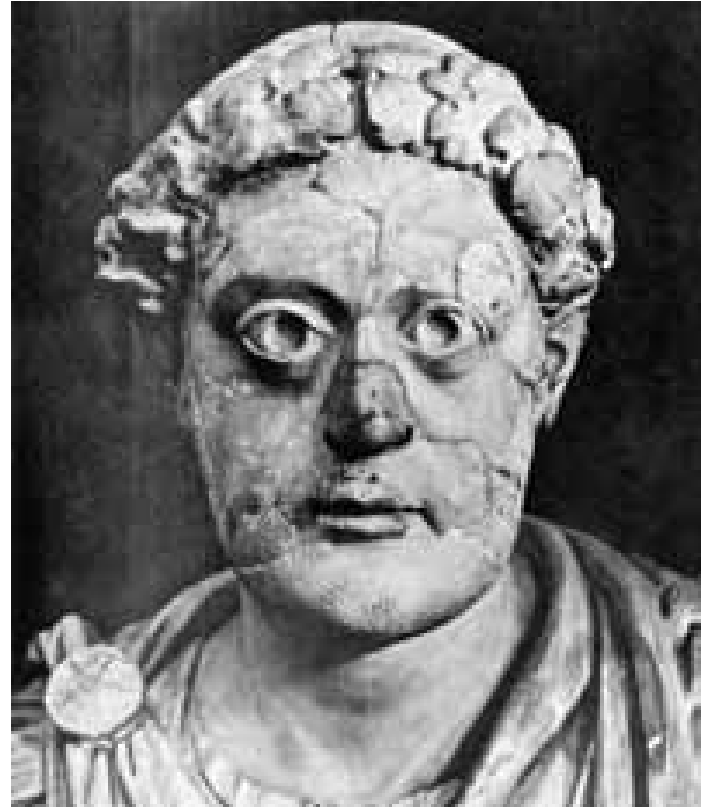

85 Parigi, Museo del Louvre: testa di alabastro

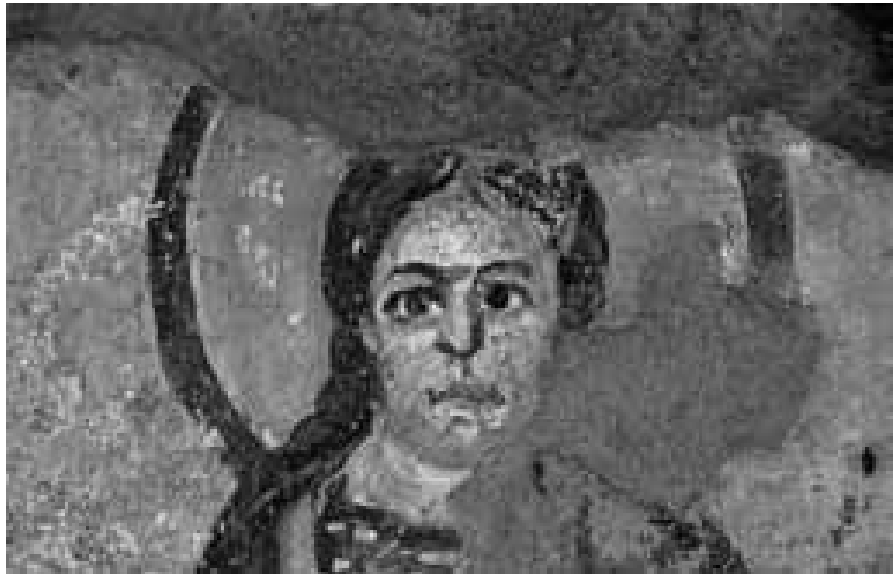

86 Roma, catacomba di Commodilla: affresco della Traditio clavium, volto di Cristo

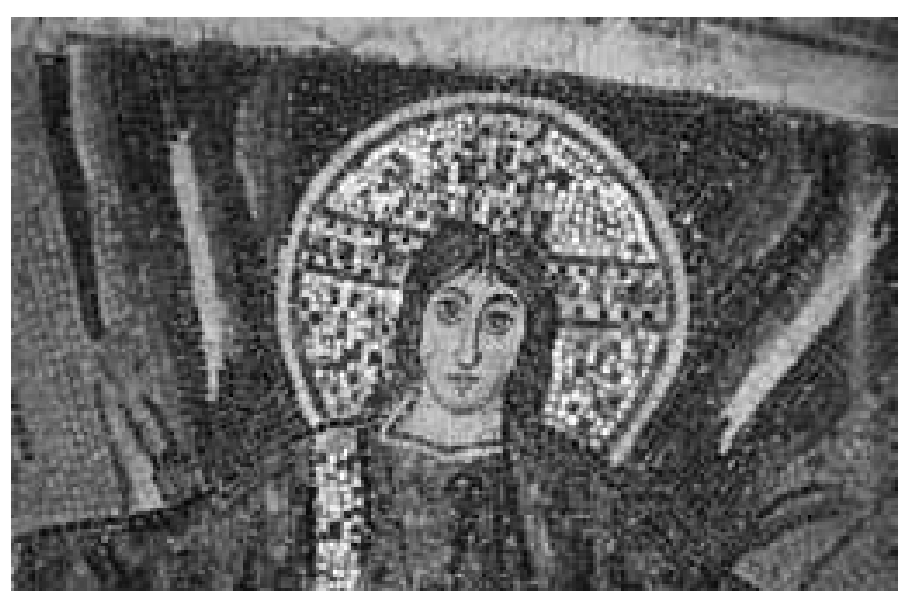

87 Parenzo, basilica eufrasiana: Cristo nell’absidiola nord

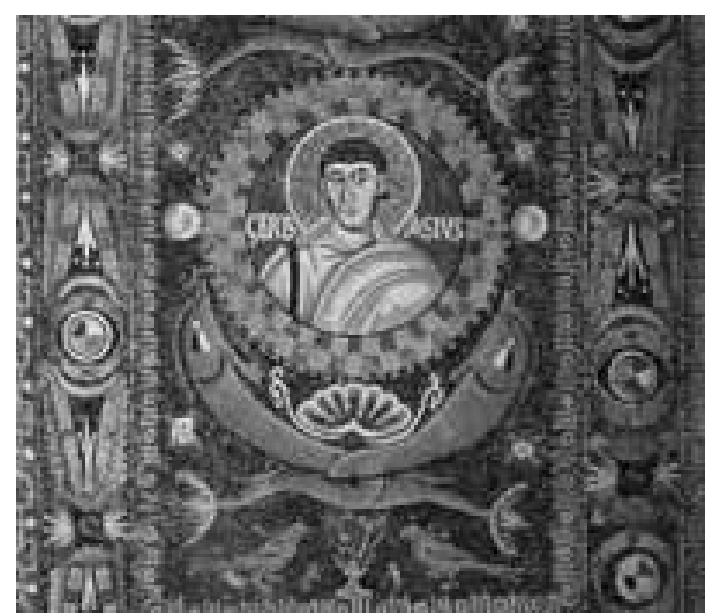

88 Ravenna, S. Vitale: S. Gervasio nel sottarco presbiteriale 


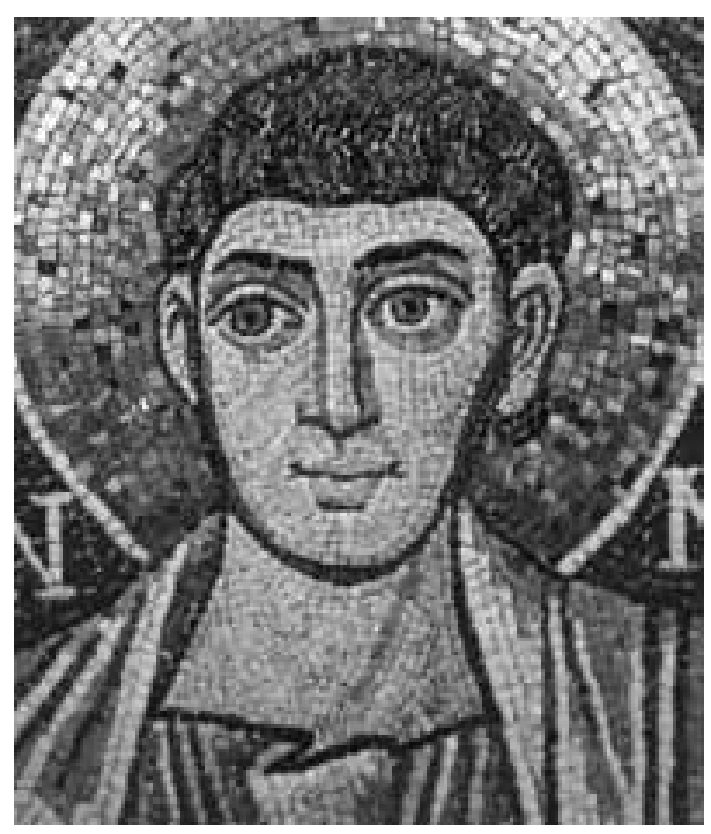

89 Ravenna, S. Vitale: S. Giovanni nel sottarco presbiteriale

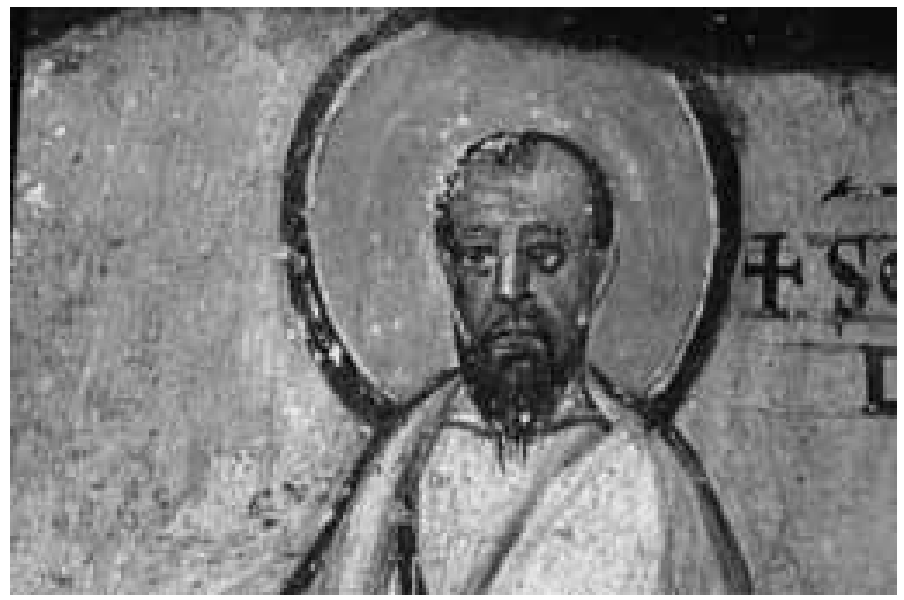

90 Roma, catacomba di Commodilla: affresco della Traditio clavium, S. Paolo

oltre che della tradizione e della funzione liturgica cui eran destinate negli edifici ${ }^{356}$, per la scelta di formati di notevole grandezza e monumentalità, e con taglio allungato ${ }^{357}$; e pure nell'applicazione della tela sopra la tavola $^{358}$ si deve vedere una concessione o un recepimento delle consuetudini romane.

Del resto le icone di Roma »sono icone al modo di Bisanzio; non sono la raffigurazione di qualcuno, ma è quel qualcuno che si presenta, appalesandosi. Guarda. Non siamo noi che guardiamo ma è lo sguardo della Theotokos, degli angeli, persino dell'offerente papa in proskynesis nella tavola di S. Maria in Trastevere che, davanti all'icona, si rivolge a noi. Lo spazio davanti all'icona è lo spazio attraversato dallo sguardo, dal riconoscimento, dal flusso psicologico legante gli sguardi $\aleph^{359}$.

Dunque la seconda metà del VI secolo si rivela in pittura complessa come la prima metà, che vedeva contrapposti il mosaico dei Ss. Cosma e Damiano e l'affresco di Maria Regina in S. Maria Antiqua: ora la compresenza di tendenze opposte ci propone l'Angelo bello e le icone di S. Maria Nova e di S. Maria in Trastevere da una parte, e il mosaico di S. Lorenzo f. l. m. dall'altra. Ma come la realtà sia complessa a S. Lorenzo f. l. m., in parallelo con quant'abbiam osservato per la scultura, ci è indicato dalle tre diverse mani e tendenze che son state còlte nel mosaico dell'arco absidale, che G. Matthiae ha distinto di formazione su modelli di Ravenna per l'autore delle teste di Cristo, degli apostoli e di s. Stefano, su modelli dell'antichità classica per l'autore della testa di s. Ippolito, »su modelli aulici bizantini del periodo giustinianeo estranei all'ambiente romano« per l'autore delle teste di papa Pelagio e di s. Lorenzo ${ }^{360}$ : e oggi, dopo le osservazioni effettuate dal gruppo di restauratori dell'Istituto Centrale del Restauro ${ }^{361}$, dobbiam prendere atto che della fase più antica del mosaico sono le teste di Pelagio, Lorenzo e Ippolito, mentre »successivamente, in una data da stabilire«, »viene realizzata la parte centrale del mosaico con le figure del Cristo e dei SS. Pietro, Paolo e

356 Vd. Russo (nota 297) 50-52. 78-82; Russo (nota 274) 142.

357 Russo (nota 297) 52; M. Andaloro in: Roma fra Oriente (nota 299) 751.

${ }^{358}$ M. Andaloro in: Roma Fra Oriente (nota 299) 751.

359 M. Andaloro in: Arte e iconografia (nota 300) 51.

${ }^{360}$ G. Matthiae, Mosaici medioevali delle chiese di Roma (1967) 154-162 tavv. XIX-XX. 89. 91-97. Cf. Andaloro (nota 353) 189-190 fig. 30; M. Andaloro in: Matthiae (nota 292) 246; C. Bertelli in: Aa. Vv., La pittura in Italia. L’altomedioevo (1994) 206. 236 nota 4 fig. 257.

361 Aa. Vv., Il mosaico pelagiano di San Lorenzo fuori le mura a Roma. Studio degli strati e dei materiali preparatori, in: I mosaici. Cultura, tecnologia, conservazione. Atti del Convegno di studi, Bressanone, 2-5 luglio 2002 (2002) 185-191 fig. 7. 
Stefano «362: ebbene, al di là dei problemi cronologici posti dalle ultime constatazioni, io ritengo comunque che le teste di Cristo, degli apostoli e di s. Stefano sian dovute ad una maestranza romana che cerca d'imitare i modi dei grecocostantinopolitani, la testa di s. Ippolito sia opera d'un artefice romano che non imita i modi dei greco-costantinopolitani, le teste di papa Pelagio e di s. Lorenzo sian frutto della presenza sul posto di mosaicisti greco-costantinopolitani (dunque con differenze pure tra le teste più antiche, Ippolito da una parte, Pelagio e Lorenzo dall'altra).

A Roma eran pertanto attive nel VI secolo botteghe che mostravano anche la compresenza di maestranze di cultura e provenienza diversa, all'opera in uno stesso cantiere.

Dopo quant'abbiam constatato per il VI secolo, non possiam certo stupirci se laboratorii di Roma, e soltanto essi, all'epoca di Gregorio Magno eseguirono le copertine dell'evangeliario conservate nel Tesoro del duomo di Monza (fig. 96) ${ }^{363}$, dono del pontefice nel 603 alla regina Teodelin$\mathrm{da}^{364}$, la quale a sua volta donò l'evangeliario alla cattedrale di S. Giovanni Battista, com'è attestato dalla scritta sovrapposta $^{365}$. Le caratteristiche della decorazione, di classica compostezza e al contempo d'uno smagliante colorismo, il suo equilibrio, l'impiego persino di rari e preziosi cammei antichi $^{366}$, non trovan confronto, neppure di lontano, con le altre opere conosciute alla corte di Monza, e non posson porre l'esecuzione in altro luogo che a Roma ${ }^{367}$. Nettissimi

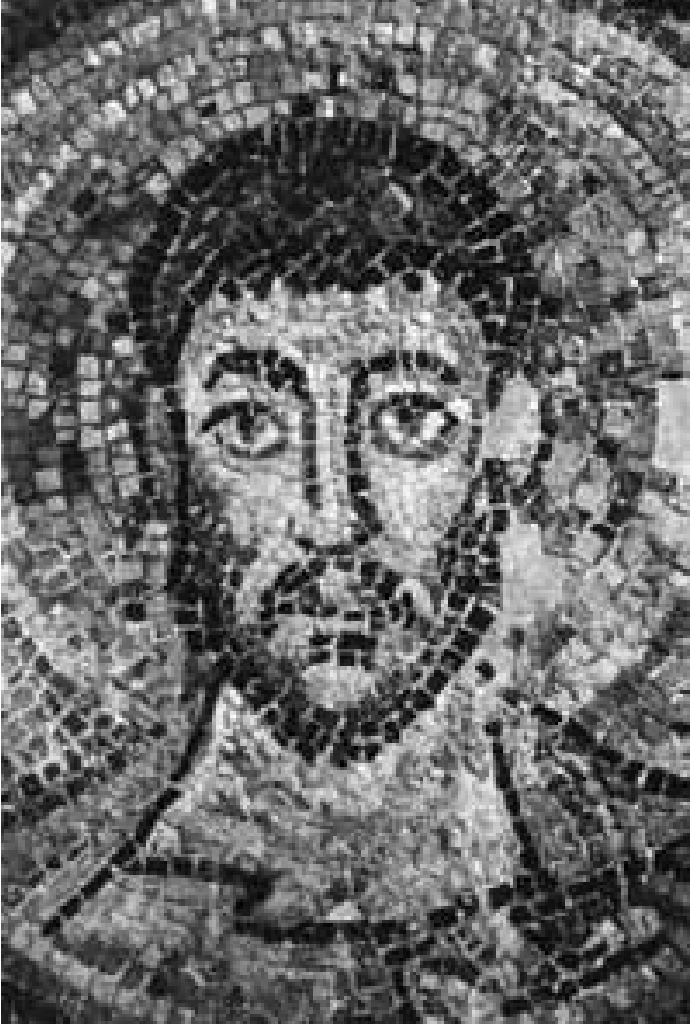

91 Lythrankomi (Cipro), Panaghia Kanakariá: S. Matteo nel mosaico absidale son gli elementi orientali, particolarmente siriaci oltre che greco-costantinopolitani, dovuti appunto ad artefici non romani, passati però attraverso la mediazione dei modi classici d'un laboratorio e d'una città come Roma.

Oggi tuttavia la critica, condizionata dal mito dei 'longobardi', tende a vedere, del tutto a torto, nelle copertine dell'evangeliario »un oggetto di committenza regia e di produzione milanese ${ }^{368}$. Gli argomenti dei sostenitori di tale divisamento si fondano sull'interpretazione della theca Persica di cui parla papa Gregorio nella sua lettera a Teodelinda come dell'»involucro di un libro fatto di stoffa persiana, vale a dire di provenienza orientale, essendo persicus un'espressione usata nel primo medioevo per indicare un tessuto blu scuro « ${ }^{369}$, oppure come di una legatura »in cuoio dipinto e dorato « ${ }^{370}$, dunque non una copertina di oro e pietre

362 Ibidem 186-187 figg. 1-3, 5.

363 A. Merati, Il Tesoro del duomo di Monza (1963) 30 figg. 28-29; D. Talbot Rice in: Aa. Vv., Il Tesoro del duomo di Monza (1966) 30-31 tavv. 45-48; Russo (nota 135) 84-86 = 108-110 (con bibl. precedente e discussione del problema); Russo (nota 297) 65-66; E. Russo in: Aurea Roma (nota 6) 199 fig. 8.

${ }^{364}$ Gregorius I Papa, Registrum epistolarum, in: P. Ewald - L. M. Hartmann (edd.), Monumenta Germaniae Historica, Epistolae (Berlin 1889) II 431; D. Norberg (ed.), Corpus Christianorum, Ser. Lat., CXLA (1982) 1083 (lib. XIV, ep. 12: dicembre 603): Excellentissimo autem filio nostro Adulouualdo regi trasmittere filacta curauimus, id est crucem cum ligno sanctae crucis Domini et lectionem sancti euangelii, theca Persica inclausum.

${ }^{365}$ De donis D(e)i offerit /Theodelenda reg(ina)/gloriosissema /s(an)c(t)o Iohanni Bapt(istae)//in baselica/quam ipsa fund(avit)/in Modicia/prope pal(atium) suum.

366 G. Flaccomio, Le gemme incise, in: Aa. Vv., Monza. Il duomo e i suoi tesori (1988) 49-51 figg. 45-50.

367 A. Lipinsky, Der Theodelindenschatz im Dom zu Monza, Das Münster 13 (1960) 159-161 figg. a pp. 152-153; W. F. Volbach in: J. Hubert - J. Porcher - W. F. Volbach, L’Europa delle invasioni barbariche (1968) 231. 364 fig. 241.

368 A. Iacobini, Aurea Roma. Le arti preziose da Costantino all'età carolingia: committenza, produzione, circolazione, in: Roma fra Oriente (nota 299) 667 fig. 27 (con bibl. precedente).

${ }^{369}$ V. H. Elbern, Riflessi della dignità imperiale di Milano nell'arte altomedievale, in: Felix temporis reparatio. Atti del Convegno archeologico internazionale »Milano capitale dell’impero romano«, Milano, 8-11 marzo 1990 (1992) 396 fig. 2.

${ }^{370}$ M. Frazer, Oreficerie altomedievali, in: Monza (nota 366) 17. 25 fig. 10. 


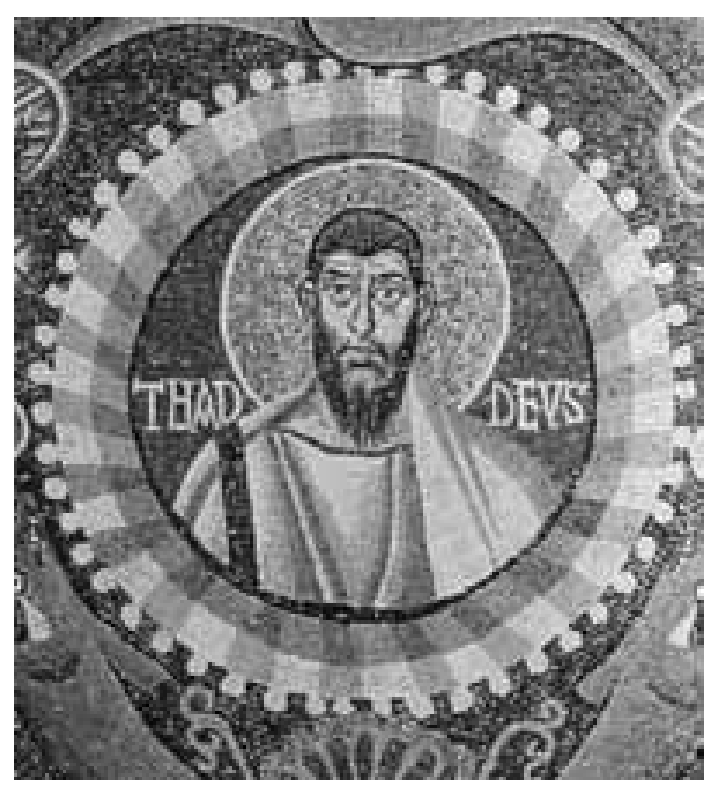

92 Ravenna, S. Vitale: S. Matteo nel sottarco absidale
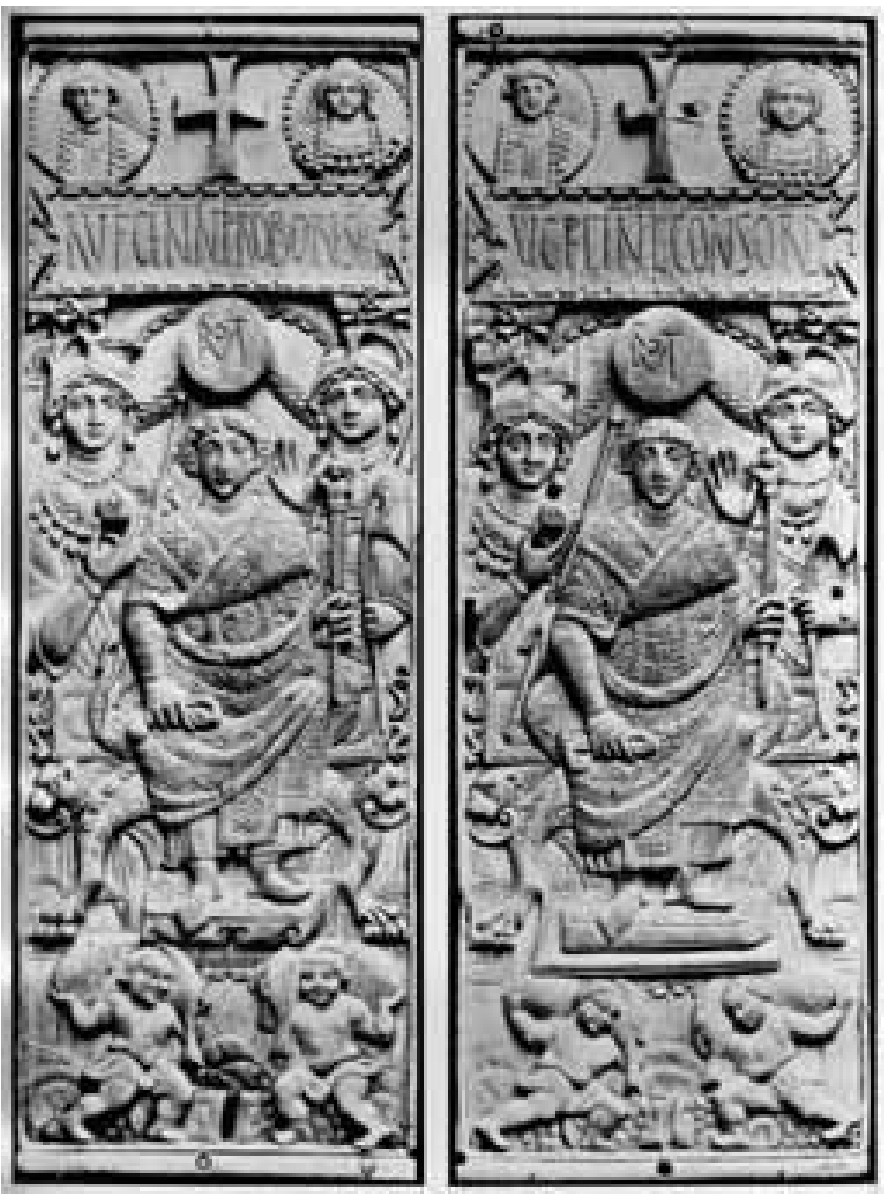

93 Londra, Victoria and Albert Museum: dittico di Oreste

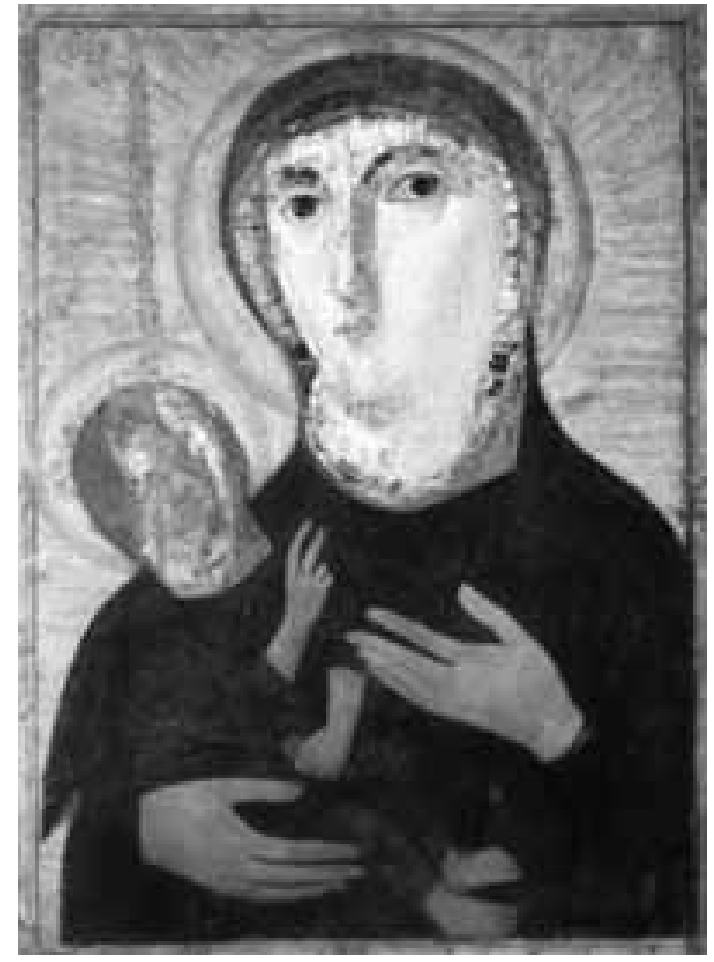

94 Roma, S. Maria Nova: icona della Madonna, volto della Madonna

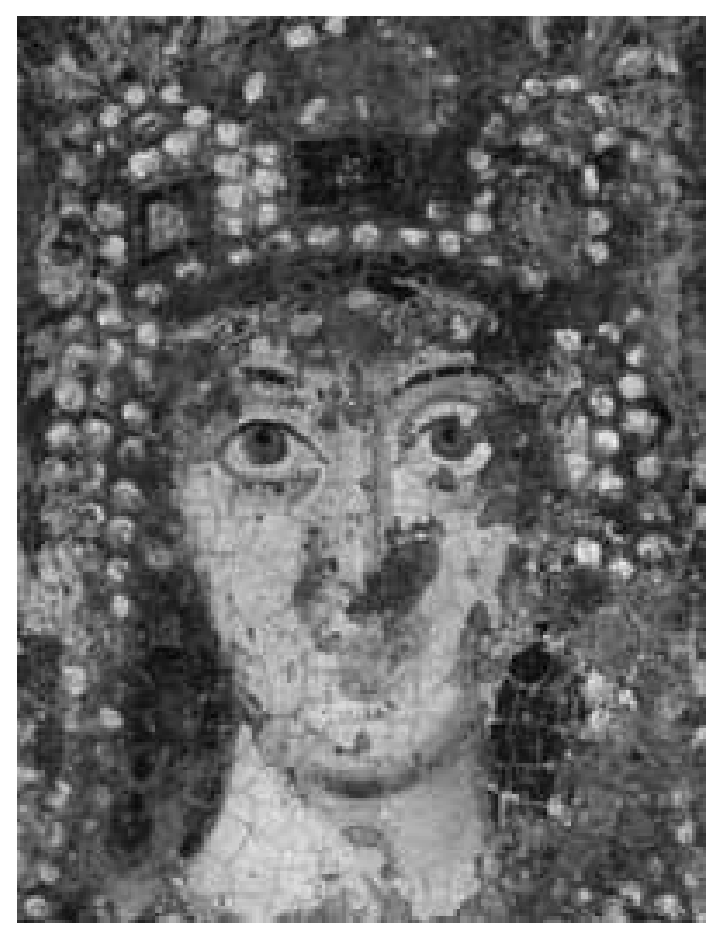

95 Roma, S. Maria in Trastevere: icona della Clemenza, volto della Madonna 
preziose; sulla delicatezza di Teodelinda che non avrebbe - a loro parere - offerto con tanto di scritta alla cattedrale un dono fatto a lei dal papa; e sulla somiglianza delle croci sulle copertine dell'evangeliario con quelle di due lastre teodelindee del duomo (figg. 97. 98) ) $^{371}$ come tipicamente longobarde ${ }^{372}$. Ques'ultimo argomento è destituito di fondamento, non essendo simili le croci ed essendo state eseguite le decorazioni delle lastre da non longobardi ${ }^{373}$. Ma pure le altre motivazioni sono connotate da estrema debolezza. V. H. Elbern ha citato, a sostegno del suo assunto, il Mediae Latinitatis Lexicon minus, però non ha letto bene, giacché il lexicon, s. v. persicum riporta due significati, il primo »couleur bleu foncé - perse, dark blue colour «, il secondo »drap bleu foncé - dark blue cloth «, ma non »nel primo medioevo" come afferma lo studioso tedesco, bensì nel XIII secolo ${ }^{374}$ : a più di 600 anni di distanza dalla lettera di Gregorio Magno, dunque usando un argomento in realtà veramente inconsistente. La Frazer a sua volta basa la sua convinzione che con theca Persica ci si riferiva a una legatura in cuoio dorato e dipinto sulle parole di K. Weitzmann a proposito del libro tenuto in mano da Cristo nella celebre icona del monastero di S. Caterina al Sinai: secondo lo studioso si tratta d'un evangeliario "with gold lines drawn around jewels and with striations filling the ground. This suggests a

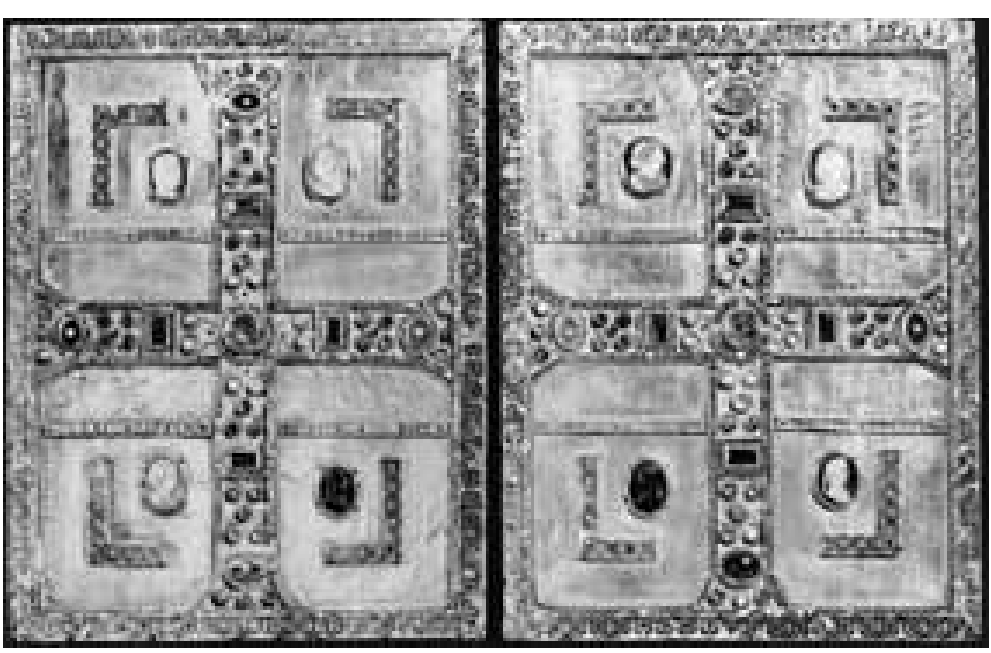

96 Monza, Tesoro del duomo: copertine di evangeliario

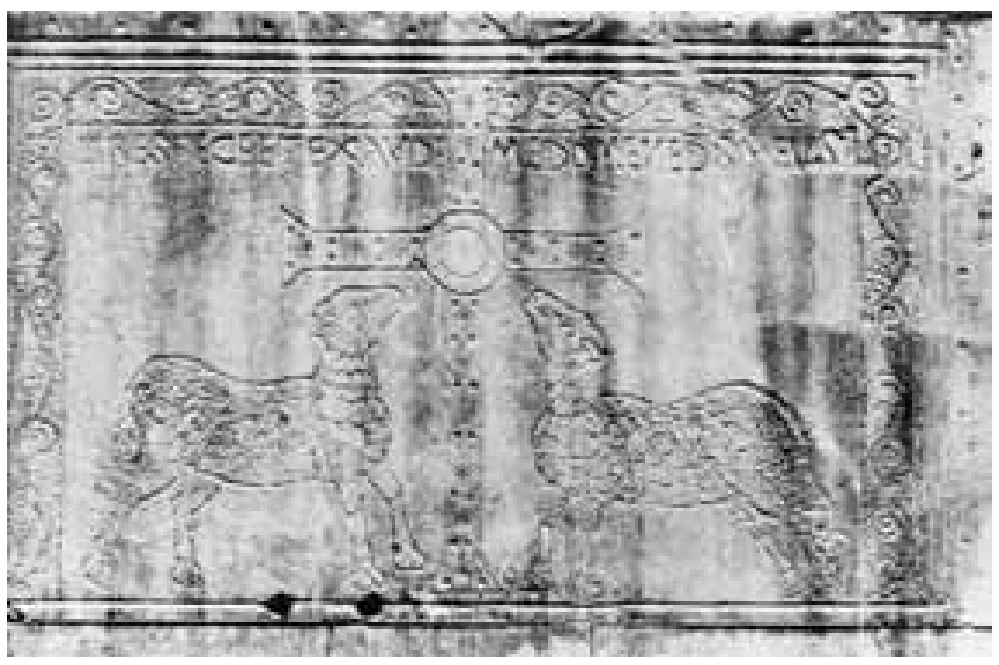

97 Monza, Tesoro del duomo: lastra dell'epoca di Teodelinda, già riadoperata nell'attuale facciata di S. Giovanni Battista pressed leather binding rather than a metal cover, and the two claps also appear to be leather. This scheme of decoration reflects that of actual contemporary bindings, as is demonstrated by a comparison with the covers of the Gospels in Monza, given to that cathedral by Queen Theodolinda in the early seventh century though here the ground is metal and not leather « ${ }^{375}$. Ma è, più ancora che inconsisten-

\footnotetext{
${ }^{371}$ Per le due lastre teodelindee del duomo vd. Russo (nota 135) 80-86 = 104-110 (con la bibl. precedente); L. Caramel, Dalle testimonianze paleocristiane al Mille, in: Storia di Monza e della Brianza IV 1 (1976) 145-148 figg. 70-71; E. Russo, L’ambone di S. Maria della Misericordia di Ancona, Atti e Memorie della Deputazione di storia patria per le Marche (Atti del Convegno »Istituzioni e società nell’alto medioevo marchigiano«) 86 (1983) 474-475; e per le immagini vd. da ultimo R. Cassanelli, Monza: il palazzo e la cappella palatina da Teodelinda a Berengario, in: Aa. Vv., »Ubi Palatio dicitur«. Residenze di re e imperatori in Lombardia (1999) figg. 3. 5.

${ }^{372}$ V. H. Elbern in: Felix temporis (nota 369) 396.

373 Russo (nota 135) 80-86 = 104-110.

${ }^{374}$ C. van de Kieft (ed.), J. F. Niermeyer, Mediae Latinitatis lexicon minus (1976) 790 s. v. persicum e 2(2002) 1030 s. v. persicum.

375 K. Weitzmann, The Monastery of Saint Catherine at Mount Sinai. The Icons I (1976) 24 tav. 1.
} 


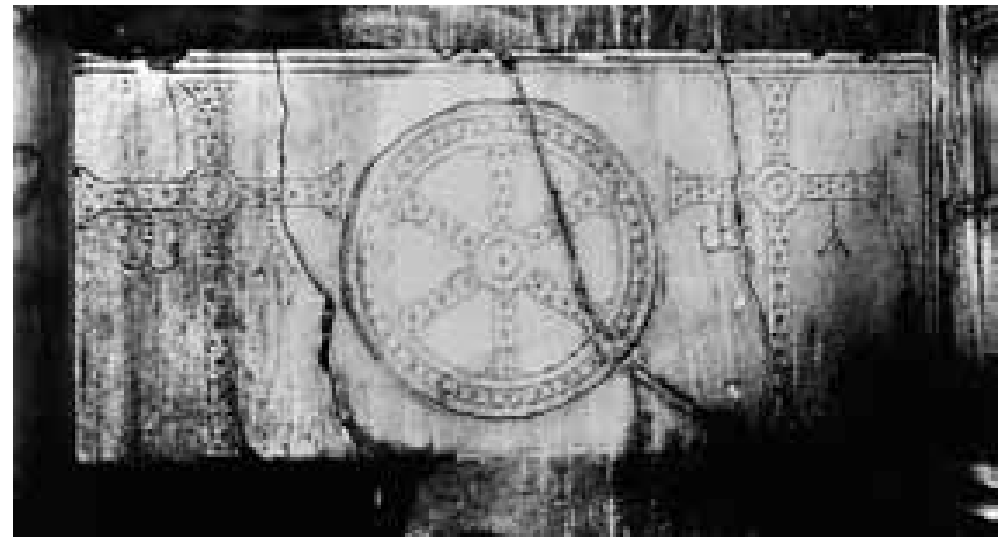

98 Monza, S. Giovanni Battista: lastra dell’epoca di Teodelinda riadoperata nell'attuale facciata te, letteralmente bizzarro inferire dalle parole di Weitzmann la probabilità che - a fronte della presenza a Monza di copertine d'evangeliario reali in metallo l'espressione theca Persica rimandi a una supposta perduta legatura in cuoio. Pare più prudente intendere theca Persica come »custodia lussuosa « ${ }^{376}$ o come »ricco di colore e ornato di pietre preziose $\ll^{377}$.

Piuttosto c'è un argomento che gli zelatori della tesi milanese non voglion considerare: nelle copertine dell'evangeliario il sottile equilibrio e l'armonia della composizione vengono turbati dall'inserimento delle otto sbarrette con la de-

dica di Teodelinda a S. Giovanni Battista (fig. 96), che a un esame accurato si rivelan un'aggiunta posteriore, fatta dopo l'esecuzione dell'opera ${ }^{378}$. Le otto sbarrette, per la loro forma imprecisa e per la maniera in cui son state fissate, senza tuttavia che venisse intaccata la decorazione o spostata la simmetria ${ }^{379}$, inserite per volontà dei barbari rompendo l'equilibrio della composizione, sono prova d'una sensibilità diversa da parte dei nuovi venuti, che avvertono il fascino della superiore civiltà con cui son a contatto, ma non riescono ad adattarsi ai suoi canoni e anzi li alterano ${ }^{380}$. È quel che accade anche nella lastra con gli agnelli della decorazione teodelindea del duomo di Monza (fig. 97): come feci notare nel lontano 1972, la cornice con girali e grappoli fu eseguita come aggiunta da una mano diversa - quella ben inferiore che incise anche l'epigrafe - rispetto all'artefice primo della decorazione. Difatti medesima è la tecnica impiegata nell'iscrizione e nella cornice a girali, mentr'essa appare del tutto differente nella restante decorazione della lastra, ch'è chiaramente improntata a un pittoricismo di marca greco-costantinopolitana ${ }^{381}$.

D’altro canto la dedica da parte di Teodelinda d'un dono del papa alla basilica di S. Giovanni Battista si spiega anche con un motivo tutto psicologico, se è vero che la regina aveva fatto sorgere la chiesa come voto e auspicio per una nuova maternità, che il nuovo nato ivi era stato battezzato ${ }^{382}$ e che Gregorio Magno precisamente per festeggiare la nascita e il battesimo aveva inviato al piccolo l'evangeliario ${ }^{383}$. Io sono convinto - e tutto converge in tale direzione - che le copertine conservate oggi a Monza sian dono di papa Gregorio Magno; ma se pur esse non facessero parte del dono del papa, non potrebbero comunque non esser assegnate a Roma come luogo d'esecuzione, dovendosi escluder del tutto Milano.

All'aprirsi del VII secolo l'evangeliario, come la recinzione gregoriana per S. Pietro in Vaticano, come l'icona del Pantheon (609) ${ }^{384}$, mettendo a frutto l'eredità del passato e grazie alla presenza delle maestranze

\footnotetext{
${ }^{376}$ Lipinsky (nota 367) 159-161; Merati (nota 363) 30.

377 D. Talbot Rice in: Tesoro (nota 363) 31.

${ }^{378}$ Lipinsky (nota 367) 159-161; Russo (nota 135) 85-86 = 109-110; L. Caramel in: Storia di Monza (nota 371) $158-159$.

${ }^{379}$ Ha a torto ritenuto contemporanei evangeliario e sbarrette, e vi ha visto pure erroneamente una decorazione »in simmetria spostata, fin dall'origine«, G. P. Bognetti, Storia, archeologia e diritto nel problema dei longobardi, in: Atti del $1^{\circ}$ Congresso internazionale di studi longobardi (Spoleto, 27-30 settembre 1951) (1952) 82.

${ }^{380}$ Russo (nota 135) $86=110$.

${ }^{381}$ Russo (nota 135) 83-84 = 107-108.

382 G. P. Bognetti, Milano longobarda, in: Storia di Milano II (1954) 127; Russo (nota 135) 86 = 110.

383 Russo (nota 135) 86 = 110. Le sbarrette inoltre, con la loro scritta, ci danno la conferma inconfutabile, per le particolarità tecniche e linguistiche, che allo stesso periodo risale anche l'iscrizione sul pluteo con gli agnelli: Bognetti (nota 379) 82; Russo (nota 135) $86=110$.

384 Russo (nota 297) 51-52. 78. 81-82 (609) (con la bibl. precedente); M. Andaloro in: Matthiae (nota 292) 255-256 (609); Amato (nota 301) 34-39 fig. 1 a p. 35 (609); Belting (nota 299) 77. 141-142 tav. di fronte al frontespizio, figg. 8. 74 (609); M. Andaloro in: Aurea Roma (nota 6) 661-662 n. 376; eadem in: Roma fra Oriente (nota 299) 723. 734. 736. 751-752. 753 figg. 3. 14; eadem in: Arte e iconografia (nota 300) 40. 53 figg. 23. 39; Osborne (nota 299) 141 (609); Thunø (nota 298) 81 fig. 4 (609).
} 
greco-costantinopolitane e orientali, è il segno d'una continuità che si nutrirà dei successivi innesti d'artefici venuti dall’Oriente nel corso del VII e dell’VIII secolo.

Prof. Dr. Eugenio Russo

Università degli Studi di Bologna, Dipartimento di Storie e Metodi per la Conservazione dei Beni Culturali, Via degli Ariani, 1, I-48100 Ravenna

Referenze fotografiche: Fig. 1: da Kapitän (nota 18) fig. 4; figg. 2. 7. 8. 9. 16. 53. 57: foto Istituto Archeologico Germanico, Roma; figg. 3. 6. 35: da Guidobaldi - Barsanti - Guiglia Guidobaldi (nota 3) figg. 38a. 60a. 300; fig. 4: da Severin (nota 48:1998) tav. 29, 4; fig. 5: da Sodini - Kolkotsas (nota 2) tav. 17d; figg. 10-13. 58: foto Istituto di Norvegia, Roma; figg. 14. 15. 51: foto Musei Vaticani; fig. 17: da Feissel (nota 88:1983) tav. 21; fig. 18: fotomontaggio da foto S.P.Q.R. e M. Fedeli; figg. 19. 20. 26. 31. 32. 43. 46. 75: foto E. Russo; fig. 21: da Guidobaldi (nota 101) fig. 2b e c; fig. 22: da Strube (nota 113:2002) tav. 96a e b; figg. 23. 24: da Brands (nota 113:2002) tavv. 91b. 93b; fig. 25: da Augenti (nota 132) figg. 10. 11; figg. 27-30: da Cozza (nota 137) figg. 12a. b. 13a. b. 14a. c. 15a. b; figg. 33. 34: da Mazzucco (nota 152) figg. 6. 5; fig. 36: incisione di G. B. Piranesi (1754); fig. 37: disegno conservato nel Cod. Vat. lat. 1379, fol. 130; fig. 38: da Séroux d'Agincourt (nota 161); fig. 39: disegno di F. Caracciolo (1826); fig. 40: da Avakian (nota 168) fig. 10; fig. 41: da Quibell (nota 181) tav. 3 in basso a sinistra; fig. 42: da Quibell (nota 182) tav. 14; figg. 44. 45. 47: da Broccoli (nota 69) tavv. 48. 50; fig. 48: fotomontaggio da foto E. Russo; fig. 49: foto S. Episcopo; figg. 50. 62-64: foto R.F.S.P; figg. 52. 54-56: foto Ch. Larrieu per il Musée du Louvre; fig. 59: da Liverani (nota 243) fig. 189; fig. 60: foto P. Lebaude per il Musée du Louvre; fig. 61: da Roma dall’antichità (nota 14) fig. a p. 222 in alto; fig. 65: foto B. M. Apollonj Ghetti; figg. 66-68: da Guidobaldi - Guiglia Guidobaldi (nota 267) figg. 80.84 tav. 2, 1; fig. 69: da P. Romanelli - P. J. Nordhagen, S. Maria Antiqua (1964) tav. 1; figg. 70. 80. 94. 95: da Andaloro in: Arte e iconografia (nota 300) figg. 26. 25. 22. 27; figg. 71. 76. 77. 87: da Prelog (nota 307:1994) tavv. 40. 5. 27. 25; fig. 73: da Kiang (nota 311) figg. 1. 2; fig. 74: da Schade (nota 298) tav. 66, 1-2; figg. 78. 84: da Poulsen (nota 319) tavv. 349. 333; figg. 79. 86. 90: da Deckers et al. (nota 297) tavv. 7 e a colori 4. 5b; figg. 81. 82. 91: da Megaw - Hawkins (nota 324) tavv. 135. 134. 139; fig. 83: da Bagatti (nota 67) fig. 100; fig. 85: da Sande (nota 309) fig. 5; figg. 88. 92: da La Basilica di San Vitale (nota 344) figg. 605. 604; fig. 89: da Racagni (nota 345) fig. 10; fig. 93: da Volbach (nota 318) tav. 16; fig. 96: da Hubert - Porcher - Volbach (nota 367) fig. 241; figg. 97. 98: foto T. Farina. 
